\title{
DEVELOPMENT AND VALIDATION OF AN ANALYTICAL CHARGE-HOLD-VENT MODEL FOR CRYOGENIC TANK CHILLDOWN
}

\author{
by \\ KEATON ANDREW KEEFER \\ Submitted in partial fulfillment of the requirements \\ for the degree of Master of Science \\ Department of Mechanical and Aerospace Engineering \\ Case Western Reserve University
}

May 2013 


\title{
Case Western Reserve University School of Graduate Studies
}

We hereby approve the thesis of

$\underline{\text { Keaton Andrew Keefer }}$

candidate for the Master of Science degree*.

\author{
(signed) Paul Barnhart \\ Joseph Prahl \\ $\underline{\text { Yasuhiro Kamotani }}$
}

(date) March 28, 2013

*We also certify that written approval has been obtained for any proprietary material contained therein. 


\section{Contents}

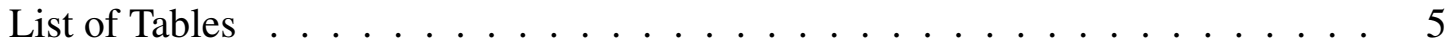

List of Figures $\ldots \ldots \ldots \ldots \ldots \ldots \ldots \ldots \ldots$

List of Symbols . . . . . . . . . . . . . . . . . . . . . 12

Acknowledgements . . . . . . . . . . . . . 13

$\begin{array}{ll}\text { Abstract } & 14\end{array}$

1 Introduction 15

1.1 Depot Architecture . . . . . . . . . . . . . . . . . . . 15

1.2 Depot . . . . . . . . . . . . . . . . . . . 19

1.3 Resupply Enabling Hardware and Experimental Setup . . . . . . . . . . . 21

1.3.1 Multi-layer Insulation (MLI) . . . . . . . . . . . . . . . . . . 24

1.3.2 Liquid Acquisition Devices (LADS) . . . . . . . . . . . . . 24

1.3.3 Transfer Line . . . . . . . . . . . . . . . . . . . . . 25

1.3.4 Spray System . . . . . . . . . . . . . . . . . 26

1.4 Fluid Transfer . . . . . . . . . . . . . . . . . . . 27

1.5 Literature Review . . . . . . . . . . . . . . . . . . . . . . . . 29

1.5.1 Charge-Hold-Vent (CHV) _ . . . . . . . . . . . . . . . . 29

1.5.2 No-Vent Fill (NVF) . . . . . . . . . . . . . . . . 30

2 Methodology 32

2.1 System Description . . . . . . . . . . . . . . . . . 32 
2.1.1 Mass Injection Process (Charge) $\ldots \ldots \ldots \ldots$. . . . . . . . . 34

2.1.2 Heat Transfer Process (Hold) . . . . . . . . . . . . . . . . . . 34

2.1 .3 Vent Process . . . . . . . . . . . . . . . . . 35

2.1.4 Receiver Tank . . . . . . . . . . . . . . . . . . . . 36

2.1.4.1 Material Properties . . . . . . . . . . . . . . 36

2.1.4.2 Geometry . . . . . . . . . . . . . 36

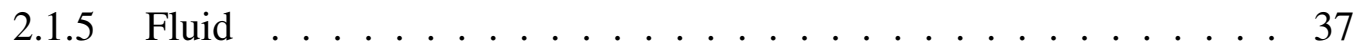

2.2 Development of Equations . . . . . . . . . . . . . . . . . . . 39

2.2.1 Control Volume Analysis . . . . . . . . . . . . . . . . . . . . . 39

2.2.1.1 Continuity: Charge . . . . . . . . . . . . . . 39

2.2.1.2 Nozzle Velocity . . . . . . . . . . . . . . . . . . 40

2.2.1.3 Continuity: Vent . . . . . . . . . . . . . . . . 41

2.2.1.4 Energy Balance: Fluid . . . . . . . . . . . . . . . 42

2.2.1.5 Energy Balance: Tank Wall . . . . . . . . . . . . . 43

2.2.2 Heat Interaction Breakdown . . . . . . . . . . . . . . . . . 44

2.3 Method of Solution . . . . . . . . . . . . . . . . . . 46

2.3.1 Multi-Variable Newton-Raphson Iteration Method _ . . . . . . . 47

2.3.2 LU Decomposition . . . . . . . . . . . . . . . . . . . . 49

2.4 Heat Transfer Model . . . . . . . . . . . . . . . . . . . 50

2.4 .1 Impinging Jet $\ldots \ldots \ldots \ldots \ldots \ldots$

2.4.1.1 Free Liquid Jet . . . . . . . . . . . . . . . . . . 52

2.4 .1 .2 Gas Jet . . . . . . . . . . . . . . . . 52

2.4.1.3 Submerged Liquid Jet . . . . . . . . . . . . . . 53

2.4.2 Flat Plate Natural Convection . . . . . . . . . . . . 55

3 Results and Discussion $\quad 59$

3.1 Data Collection . . . . . . . . . . . . . . . . . 59

3.2 Model Development based on Cycle 5 . . . . . . . . . . . . . . . 69 
3.2 .1 Tank ...................... 70

3.2.1.1 Material ................ 71

3.2.1.2 Heat Leak and MLI Model . . . . . . . . . . . . 73

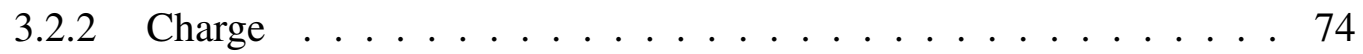

3.2 .3 Hold . . . . . . . . . . . . . . . . . . . . 87

3.2 .4 Vent ......................... 87

4 Conclusions $\quad 101$

4.1 Recommendations . . . . . . . . . . . . . . . . 102

$\begin{array}{ll}\text { Bibliography } & 106\end{array}$ 


\section{List of Tables}

2.1 Thermodynamic Properties available through REFPROP. . . . . . . . . . . 39

3.1 Cycle 5 experimental history. . . . . . . . . . . . . . . . 69

3.2 Tank geometry. . . . . . . . . . . . . . . . 71

3.3 Empirical heat leak equation parameters. . . . . . . . . . . . 73

3.4 Specified Model Inputs for Simulation of Cycle 5. . . . . . . . . . . . 98

3.5 Specified Model Inputs for Simulation of Cycle 4 followed by Cycle 5 . . . 100

4.1 Comparison of cycle 5 experimental parameters to cycle 5 simulation pa-

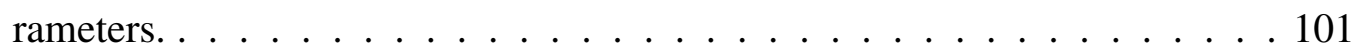




\section{List of Figures}

1.1 Potential depot configurations. Figure courtesy of [1]. . . . . . . . . . . 19

1.2 Illustration of flight hardware experimental setup. . . . . . . . . . . 21

1.3 The influence of Liquid Acquisition Devices on fluid position and liquid delivery. . . . . . . . . . . . . . . . . 25

2.1 The interaction of mass and energy with the system's control volumes. . . . 32

2.2 Examples of oblate and prolate hemispheroids at two different height to width ratios. . . . . . . . . . . . . . . . . . . . 37

2.3 Jet diagram with arbitrary orientation of inlet nozzle. . . . . . . . . . 51

2.4 Regions of natural convection heat transfer. . . . . . . . . . . 55

3.1 Test tank in testing structure [14]. . . . . . . . . . . . . . . . 59

3.2 Simplified facility schematic $[8] \ldots \ldots \ldots \ldots \ldots$

3.3 Top inlet spray nozzle. . . . . . . . . . . . . . . . . . 61

3.4 Bottom turbulent jet inlet nozzle. . . . . . . . . . . . . . . . . 62

3.5 Test tank nozzle and diode arrangement $[8] \ldots \ldots \ldots$. . . . . . . . . . 64

3.6 Temperature history for beginning stages of cycle $5[8] \ldots \ldots \ldots$

3.7 Temperature history for the 6 cycle liquid hydrogen $\mathrm{CHV}$ followed by a

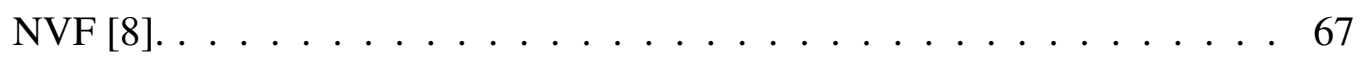

3.8 Pressure history for the 6 cycle CHV followed by a NVF [8]. Cycle 5 occurred between the 70 and 80 minute mark. . . . . . . . . . 68 
3.9 Material specific heat versus temperature. . . . . . . . . . . . 72

3.10 Simulation jet configuration. . . . . . . . . . . . . . 74

3.11 Velocity transition function. . . . . . . . . . . . . 77

3.12 Pressure history compared against jet velocity history without an imposed transition function. . . . . . . . . . . . . . . 79

3.13 Pressure history compared against temperature history without an imposed transition function. . . . . . . . . . . . . . 80

3.14 Diagram of experimental instrumentation and hardware inside the test cham-

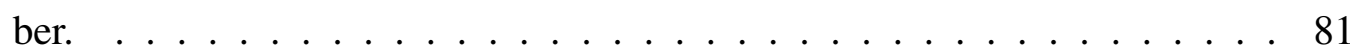

3.15 Pressure history compared against jet velocity history influenced by an imposed transition function. . . . . . . . . . . . . . 83

3.16 Pressure history compared against tank wall and fluid temperature histories influenced by the functional behavior imposed on jet velocity. . . . . . . . 84

3.17 Jet impingement and natural convection heat transfer histories. . . . . . . . 85

3.18 Location of transition time scale relative to injection time scale and jet heat transfer region. . . . . . . . . . . . . . . 86

3.19 Tank geometry approximated as flat plates for use in natural convection heat transfer model. . . . . . . . . . . . . . . . . . . . 87

3.20 Pressure and temperature histories when mass is vented instantaneously. . . 89

3.21 Pressure and temperature histories with use of sub-vents and transition function. .............................. 90

3.22 Venting transition function. . . . . . . . . . . . . . . 91

3.23 Example of parent vent behavior when varying $m_{o}^{1}$. . . . . . . . . . . 92

3.24 Example of parent vent behavior when varying the duration of the sub-vents. 93

3.25 Example of one parent vent divided into five sub-vents. . . . . . . . . . . 94

3.26 Simulation behavior for cycle 5 as a result of only natural convection effects. 95

3.27 A complete model of cycle 5 data from [8]. . . . . . . . . . . . . 96 
3.28 Simulation results compared to cycle 5 data with $7 \%$ error bars. . . . . . . . 97

3.29 A complete model of cycle 4 and cycle 5 data from [8]. . . . . . . . . . . 99 


\section{List of Symbols}

$a$

$A$

C

$C_{D}$

$C_{p}$

$C_{R}$

$C_{S}$

$C_{w}$

$D_{j e t}$

$D_{h}$

$D_{n}$

E

$f$

$F_{1}$

$g$

$G$

Gr

$h$

$h_{t o p}$

$h_{c y l}$

$h_{\text {bot }}$

$h_{\text {jet }}$

$H_{\text {jet }}$

$k$

$l$ element of partial derivative matrix, speed of sound

area, crossectional flow area, or partial derivative matrix

specific heat of non-vapor substance

discharge coefficient

specific heat at constant pressure

empirical coefficient of radiation heat transfer

empirical coefficient of solid conduction heat transfer

specific heat of tank wall material

diameter of area impinged by fluid jet

hydraulic diameter

nozzle diameter

major to minor axis ratio

iteration function, constituent mass fraction, Hemholtz free energy

subfunction of Nusselt number correlation

gravitational constant, gibbs free energy

subfunction of Nusselt number correlation

Grashof number

specific enthalpy

natural convection coefficient of top tank endcap

natural convection coefficient of cylindrical tank body

natural convection coefficient of bottom tank endcap

forced convection coefficient of jet impingement heat transfer

distance from nozzle to impingement area

thermal conductivity

element of lower triangular matrix 


\begin{tabular}{|c|c|}
\hline$L$ & length or lower triangular matrix \\
\hline$m$ & amount of mass in receiver tank \\
\hline$m_{i}$ & amount of mass injected into receiver tank (charge size) \\
\hline$\dot{m}_{i}$ & average mass flow rate into receiver tank \\
\hline$m_{o}$ & amount of mass removed from receiver tank in one sub-vent \\
\hline$m_{o}^{1}$ & $\begin{array}{l}\text { constant scaling factor for determining the magnitude of } m_{o} \text { for each } \\
\text { sub-vent }\end{array}$ \\
\hline$m_{o, t o t}$ & amount of mass removed from receiver tank by parent vent \\
\hline$m_{0}$ & pre-burn mass of spacecraft \\
\hline$m_{1}$ & post-burn mass of spacecraft \\
\hline$n$ & system of equations size \\
\hline$N_{S}$ & number of insulation sheets \\
\hline $\bar{N}$ & insulation layer density \\
\hline$N_{\text {vent }}$ & number of parent vents per $\mathrm{CHV}$ cycle \\
\hline$N_{\text {sub-vent }}$ & number of elapsed sub-vents \\
\hline$N_{\text {sub-vent }, \max }$ & total number of sub-vents composing a parent vent \\
\hline$N_{\Delta t / s u b-v e n t}$ & number of timesteps in hold phase per sub-vent \\
\hline$N_{\Delta t / h o l d}$ & number of timesteps in hold phase between parent vents \\
\hline$N_{S}$ & number of insulation sheets \\
\hline$P$ & receiver tank pressure \\
\hline$P_{a}$ & ambient pressure \\
\hline$P^{0}$ & pressure of reciever tank before a $\mathrm{CHV}$ cycle begins \\
\hline $\operatorname{Pr}$ & Prandtl Number \\
\hline$q$ & heat transfer per unit area \\
\hline$\dot{Q}_{w}$ & heat transfer between fluid contents and tank wall \\
\hline$\dot{Q}_{j e t}$ & $\begin{array}{l}\text { forced convection heat transfer between impinging nozzle jet and } \\
\text { tank contents }\end{array}$ \\
\hline
\end{tabular}




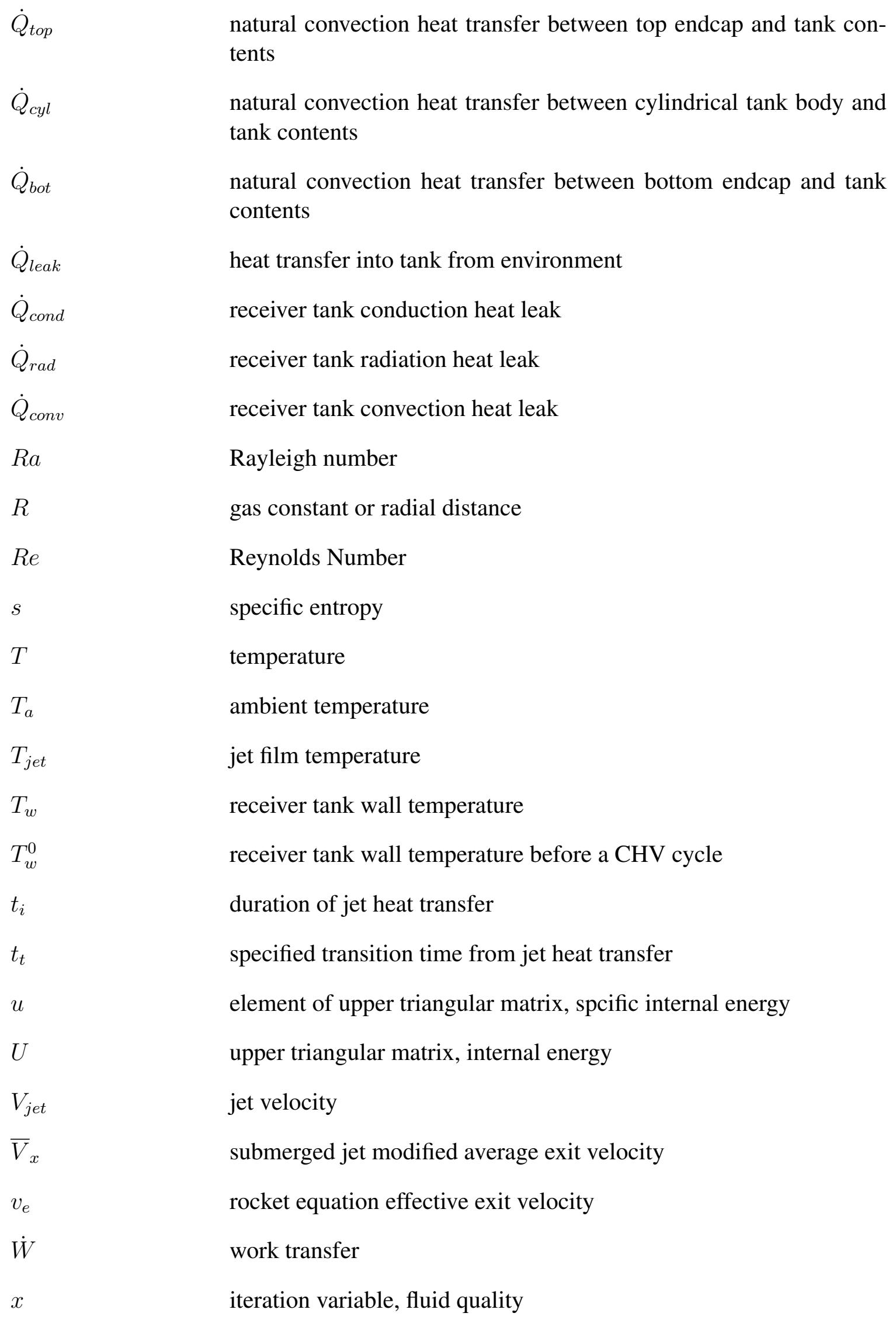




\section{Greek}

$\alpha$
$\beta$
$\delta$
$\epsilon$
$\theta_{j e t}$
$\mu$
$\xi_{0}$
$\rho$
$\sigma$
$\tau_{t}$
$\tau_{o}$

\section{Subscripts}

$i$

$j$

$k$

$l$ temporary iteration vector or element of temporary iteration vector REFPROP fluid composition array

time step size

temperature difference between receiver tank wall and fluid contents change in velocity calculated from rocket equation correction size, correction size vector, or element of correction size vector

thermal diffusivity

volumetric thermal expansion coefficient

differencing factor

emissivity or a small number

fluid jet half angle

dynamic viscosity

height to width ratio of spheroid

density

Stephan-Boltzmann constant

dimensionless transition time from jet impingement heat transfer dimensionless time during a parent vent

general index

general index

general index

general index 


\section{Acknowledgements}

I would like to thank my adviser, Dr. Paul Barnhart, for his guidance and patience during the course of my thesis work. His experience and insight provided me with a wealth of information during the development of the program.

I thank my committee members, Dr. Joseph Prahl and Dr. Yasuhiro Kamotani for their suggestions and critiques that helped to better my work and improve my understanding of the problem's underlying physics.

I thank Dave Chato for his support through the Lewis Educational and Collaborative Internship Program and for his guidance and encouragement.

I thank Jason Hartwig for mentoring me to be a better engineer and person. The discussions we had helped enrich my understanding of cryogenics and improved me as a researcher.

Lastly, I thank my parents for their constant love and support. Thank you for allowing me to pursue my desires and for providing stability and focus to my life. 
Development and Validation of an Analytical Charge-Hold-Vent Model for Cryogenic

Tank Chilldown

Abstract

by

\title{
KEATON ANDREW KEEFER
}

\begin{abstract}
A transient Charge-Hold-Vent (CHV) analytical model has been developed and validated with hydrogen experimental CHV data. The model was found to be within $7 \%$ agreement of the data. The model simulates natural convection and jet impingement heat transfer interactions using quasi-unsteady steps in time and tracks the fluids thermodynamic behavior through REFPROP. The additional cooling potential made available through the cyclic venting technique is calculated in the code. The model can simulate multiple CHV cycles and provide predictions on the amount of fluid mass used for each cycle. The impact of varying tank geometry, nozzle position, and fluid type may be simulated and considered when planning future $\mathrm{CHV}$ tests.
\end{abstract}




\section{Chapter 1}

\section{Introduction}

\subsection{Depot Architecture}

The cost of space travel and exploration has historically been very high and launch/landing schedules very strict. Improving the flexibility of launch/landing windows offers the potential for cost savings by providing more convenient options to the mission designers. One strategy for increasing flexibility in launch schedules is to distribute the mission payload among smaller and lighter launches instead of a single heavy launch. This allows the smaller payloads to be launched at separate convenient times and held in Low Earth Orbit (LEO) until the entirety of the mission architecture can be assembled. The fuel depot is a solution that may improve mission flexibility, lower costs, and push the frontier of space by allowing fuel payloads to be distributed among smaller launches. A mission architecture that incorporates the capability to refuel permits a higher payload mass and higher transfer velocities which reduce mission duration, an important factor for manned missions.

Every kilogram of payload requires a proportional amount of propellant to carry it into space. The Tsiolkovsky rocket equation, Eqn 1-1, shows the effects of carrying excess 
propellant as payload instead of burning it all during departure/arrival.

$$
\Delta v=v_{e} \ln \left(\frac{m_{0}}{m_{1}}\right)
$$

Assuming a fixed effective exhaust velocity, $v_{e}$, and initial total mass, $m_{0}$, a rocket that does not expend all on board propellant will have a larger final total mass, $m_{1}$, and smaller

mass ratio, $\frac{m_{0}}{m_{1}}$, compared to a rocket that does expend all on board propellant. The second rocket will be capable of a larger velocity change which is desirable for distant missions or high velocity transfer orbits. Fuel depots will allow for the use of all on board propellant mass with a planned propellant resupply later in the mission.

A large amount of propellant is spent launching from Earth's surface and parking in LEO. Additionally, more fuel must be spent for each kilogram of useful payload or excess fuel added on the launchpad. Excess fuel is necessary for accelerating the entire mission into/out of its transfer orbit. Under current launch system designs the fuel used for a transfer burn must be carried on board the space vehicle during its ascent to LEO. Chandler [1] compares the impact a propellant depot architecture has on the NASA Exploration Systems Architecture Study (ESAS). It was found the Earth Departure Stage expends $\approx 55 \%$ of its propellant just getting the Lunar Access Module to LEO. The amount of mass delivered to the Moon may be increased by as much as $183 \%$ by refueling the propellant spent getting to LEO. NASA plans to capitalize on fuel depots during its future lunar exploration missions and high energy transfer missions. Chandler continues with a quote from Dr. Michael Griffin from the $52^{\text {nd }}$ annual American Astronautical Society Meeting in November 2011, "But if there were a fuel depot available on orbit, one capable of being replenished at any time, the Earth departure stage could after refueling carry significantly more payload to the Moon... at a conservatively low government price of $\$ 10,000.00 / \mathrm{kg}$ in LEO. 250MT of fuel for two missions per year is worth $\$ 2.5 \mathrm{~B}$, at government rates.” The interest in depot based missions exists within the government but the initial investment in key enabling technolo- 
gies is discouraging and will require a philosophical transition away from the heavy lift ideology.

The depot architecture will not just increase the deliverable mission payload mass but also prove helpful in extending the range of exploration efforts. For example, a Mars mission can be developed from the lunar mission with effective use of depots. According to Zegler [2] a step-wise and evolutionary approach to maturing technology minimizes risks down to smaller and less costly increments. A depot strategy facilitates a modular exploration mission strategy which will help lower the total risk of the mission. Small steps in maturing the depot technology will lead to small steps in expanding the manned space frontier while reducing risks and cost.

NASA missions will not solely benefit from a depot network. Depots positioned in LEO can benefit the private companies interested in accessing LEO for profit as well as the Department of Defense (DoD) and their various missions to low earth, geostationary, and polar orbits. Private industry now has access to space and several plans to capitalize on this new market are already underway. Bigelow Aerospace has plans to station habitats in LEO and SpaceX has already successfully resupplied the International Space Station (ISS). The inclusion of a depot architecture in either of these missions would allow for both companies to be more ambitious with their mission planning. The need for on orbit flexible fluid delivery and storage is only going to grow.

The DoD may also leverage a depot network in positioning a small satellite in geostationary orbit. The satellite can be launched on a smaller and more simple rocket with more consideration towards payload safety due to the absence of station keeping fuel in the satellite. Once in orbit the satellite can purchase fuel from a depot. After refueling, the satellite positions itself in its final geosynchronous orbit avoiding the need for the launch vehicle to attain a geosynchronous orbit and providing further savings on mission cost [2].

Zegler states, "the bulk of the mass for exploration is a commodity substance with little intrinsic value." He explains that when dealing with heavy lift architectures $70 \%$ of the 
mass delivered to LEO is propellant. Propellant depots can supply the propellant necessary for exploration once the spacecraft is in LEO. This will decrease the wet mass of the craft on the launchpad and increase the useful mass to LEO. The amount of propellant provided to the vehicle would be dependent on the mission. The task of ferrying refill propellant to the depots can be opened to the private sector. Competition amongst companies would further drive down the price or propellant on orbit. The launch vehicle used for propellant depot resupply could be engineered to "absolute simplicity" because the launcher does not need to be man rated and propellant is of low value relative to human lives.

The nation should not pride itself on having the capability of heavy lift. Heavy lift rockets cannot compare to an integrated launch system utilizing a depot architecture. A new perspective on space travel will move the nation away from brute-force methods that are deliberately avoiding innovation for the sake of using previously designed and tested hardware. In the end money will be spent building a launch system that runs the risk of being obsolete on its first day. Heavy lift rockets are only useful for performing a small set of specific exploration tasks [2].

Kutter stresses the modular aspect of depots and the involvement of the market in [3]. The storage of propellant on orbit allows for resupply to take place whenever it is convenient. This allows propellant depot resupply missions to be shared payloads with satellites, ISS resupply, or any other launch where payload space is available. The ability to launch depot resupplies in cooperation with other launches will drive down the cost of the resupply mission.

Zegler [2] summarizes an effective depot architecture by explaining its role in facilitating the collection of derelict space vehicles. It was never desirable to abandon mission components once their usefulness expires but an option for "garbage collection" was never available. Dealing with the problem of accumulating mass in orbit will increase propellant demand to move these objects back to Earth. The old spacecraft may be removed by utilizing the rocket used to deliver the new spacecraft. However, the rocket will need ad- 
ditional propellant supplied to it after its delivery is made. A depot network could provide the fuel in flexible quantities to suit the different types of delivery rockets. The requirement for high $\Delta v$ is unavoidable for this type of mission. Propellant must be used to approach and rendezvous with the targeted vehicle and finally a large burn will return the rocket and payload back to Earth. However, this approach offers significant cost savings compared to launching a purpose built rocket from the Earth's surface with enough propellant on board to complete the same job. By exploiting rockets from other missions coupled with a depot resupply the idea of cleaning up satellites no longer in operation is much more attainable.

\subsection{Depot}

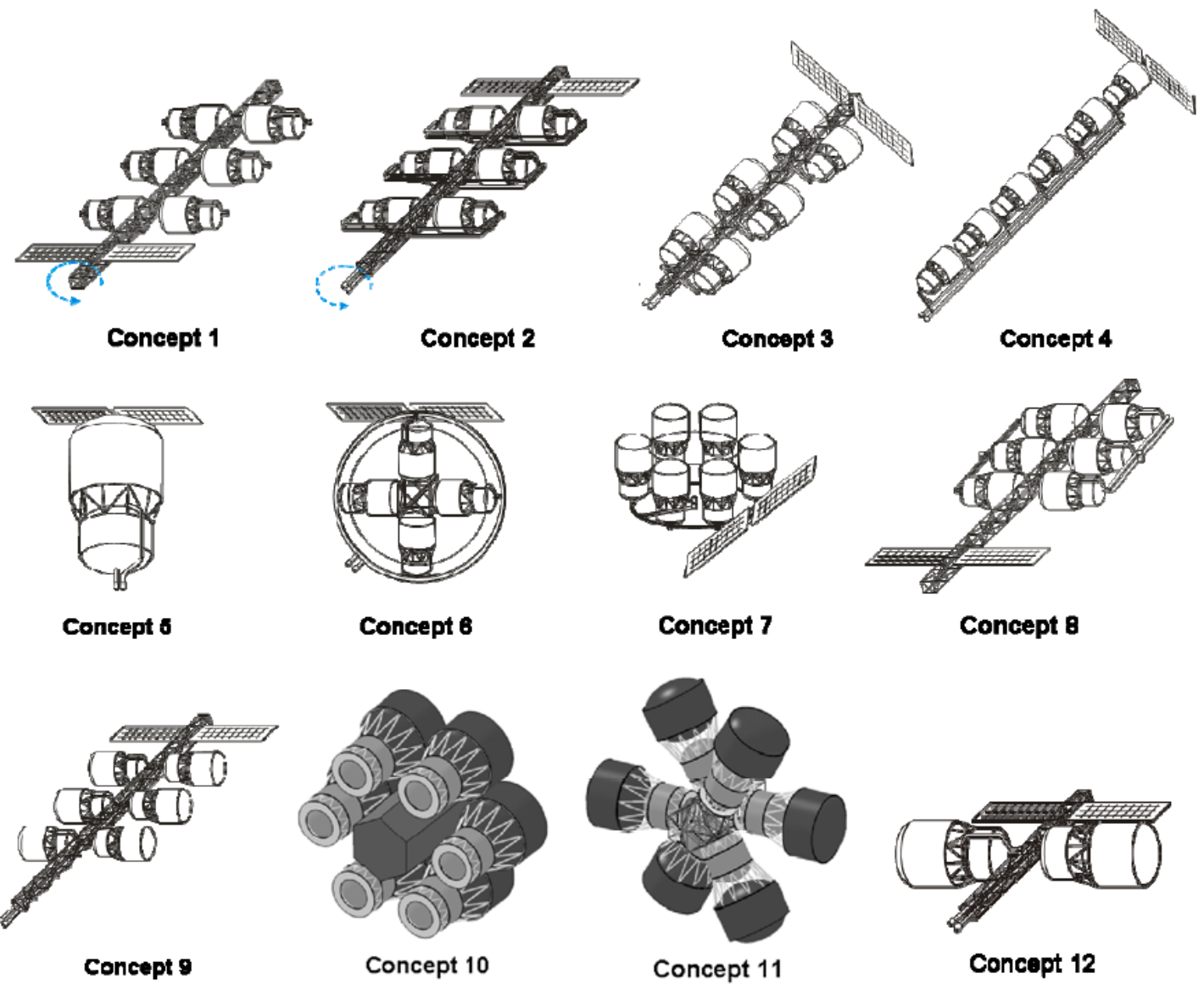

Figure 1.1: Potential depot configurations. Figure courtesy of [1]. 
Fluid depots as a general system must deal with the conditions of space. Low gravity and drastically varying hot/cold environments necessitate specific design considerations. The depot must remain lightweight so as to not burden its launch vehicle and it must remain compact so as to fit within the geometric constraints of the launch vehicle. The vehicles that dock and interact with the depots must have on board tanks that implement much of the same technology as the depot tank. Spray systems, liquid acquisition devices, and insulation systems are three main components that help single phase liquid to be transferred and stored in the environment of space.

The depot must contain hardware and software to allow space vehicles to interact with it. Chandler summarizes an interaction between a space vehicle and fuel depot.

Once the depot is ready for operation, client vehicles can be launched using conventional launch vehicles of the necessary lift capacity for the payload. Using a depot allows additional mass into orbit by letting the payload be launched without any propellant and by permitting the transfer stage, typically the launch vehicle 2 nd stage, to have its propellant load replenished at the depot. The transfer stage will be required to include mating and fluid transfer receptacles [and hardware and software] to allow it to rendezvous with the depot. Once at the depot, the transfer stage will perform the rendezvous and proximity operations necessary to dock with the depot, where the depot systems will then transfer the necessary fluids to the transfer stage. Once the fluid transfer is complete, the transfer stage will separate from the depot and move to a safe distance from the depot to then perform the required propulsive impulses to place the payload in the final mission orbit location. The transfer stage then may or may not perform a disposal burn depending on the mission specifications. 


\subsection{Resupply Enabling Hardware and Experimental Setup}

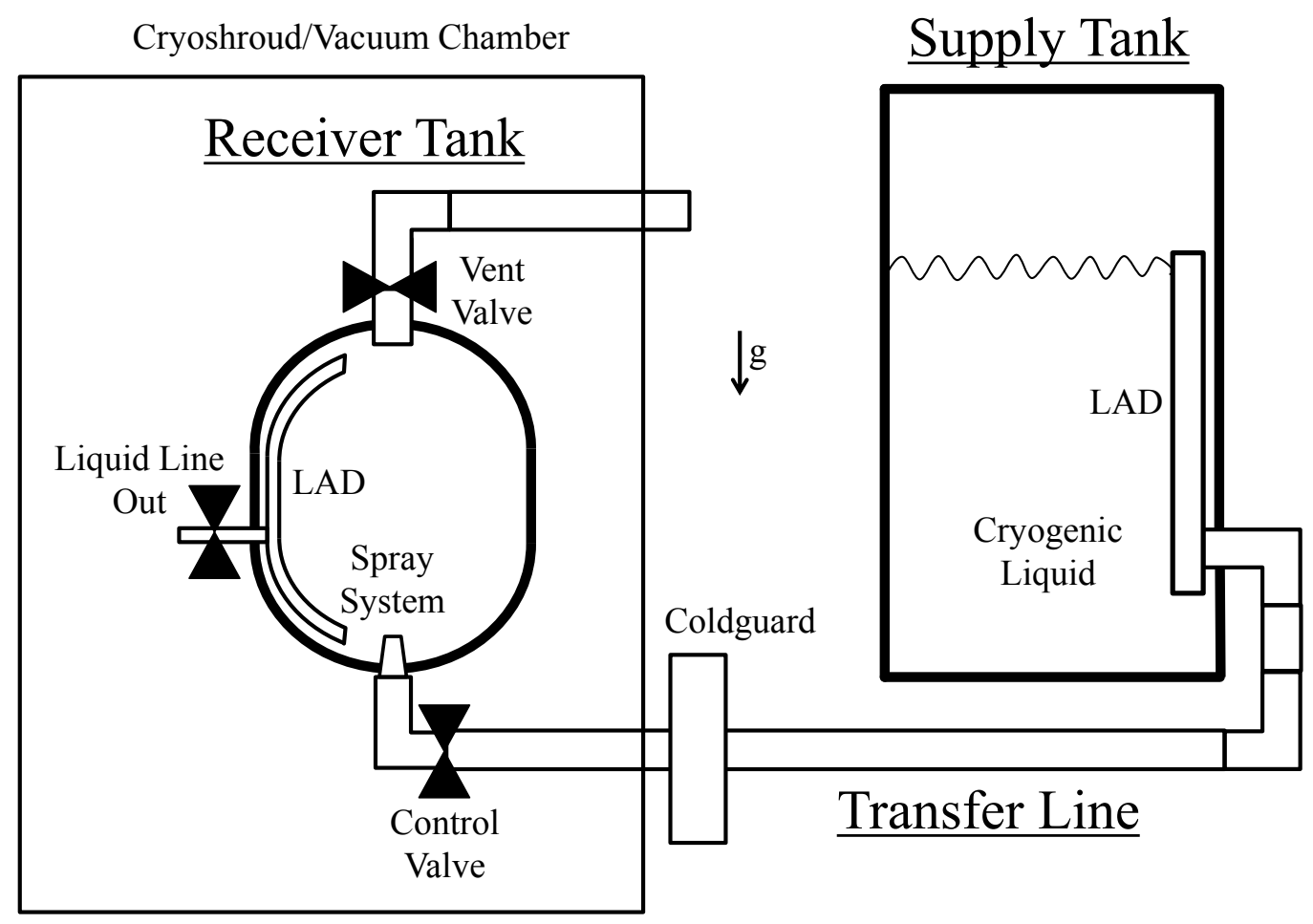

Figure 1.2: Illustration of flight hardware experimental setup. Flight hardware must be investigated under terrestrial gravity before a low gravity demo can occur.

Before a depot can ever fly extensive ground testing of its components and procedures must first occur. Fig 1.2 illustrates an experimental set up for testing cryogenic fluid transfer flight hardware. The experimental setup is outfitted with the necessary components to permit single phase liquid transfer in low gravity. The receiver tank can be thought of as the fuel tank on a satellite or spaceship while the supply tank can be thought of as the depot. Saturated liquid cryogen exists in the supply tank. The transfer line is regulated with a control valve to allow specification of flow rates and permits mass to transfer between the supply and receiver. Any mass not required by a connecting vessel may be removed from the receiver tank using the vent valve. Liquid Acquisition Devices (LADs) are fitted in both the supply and receiver tank to allow tanks in low gravity environments to be emptied of 
liquid without ingesting vapor. The liquid line out permits any connecting vessel access to liquid in the receiver tank once the transfer from the supply tank has terminated.

Ground based testing of future flight hardware is often conducted in a space-like environment. Two pieces of experimental hardware, a cold guard and cryoshroud, enable the temperatures of space to be imposed on the test system while a vacuum chamber approximates the pressure of space. The coldguard thermally shorts the hardware leading to the receiver tank. The coldguard decreases the thermal loads experienced by the experimental receiver tank due to conduction through the transfer line and instrumentation wires. The coldguard is normally filled with cryogenic liquid boiling at near atmospheric pressure to ensure the hardware is acceptably cold. The cryoshroud provides a uniform heat transfer environment during testing and can simulate temperatures seen in warm and cold Low Earth Orbits (LEOs), the likely position of early fuel depots.

All hardware external to the supply tank must be cooled to cryogenic temperatures for a liquid transfer to complete successfully. This includes the transfer line, receiver tank, and receiver tank LAD. A chilldown is deemed successful if the receiver tank can be filled without the need to vent during or after the fill process. The inability to preferentially vent liquid in a low gravity environment drives the need to preserve this constraint during ground based testing.

The transfer line, receiver tank LAD, and receiver tank possess thermal inertias that create problems when attempting to preserve the cryogen as a liquid. Mechanical devices may be implemented to cool these components before attempting to fill the receiver tank but is often not a desirable solution. Mechanical components introduce additional risks, in the form of mechanical failure, and additional weight. Another option is to cool the hardware by spending some of the liquid cryogen. Cooling the hardware by spending some of the cryogen is a viable option because it mitigates the need for additional hardware. However, the liquid used to cool the system will be lost as it will be vaporized with no way to condense it back to the liquid state. It is necessary to extract as much cooling potential 
as possible from the fluid in order to minimize the amount spent on chilldown.

The largest thermal mass is contained in the receiver tank which is expected to require the longest time until chilldown is achieved. The lines, LADS, vent valve, and spray system are sized to deliver and remove mass quickly but heat requires time to interact and establish thermal equilibrium with the hardware. The most cooling potential can be extracted from the fluid by developing a process of mass injections into the sealed tank followed by a hold period to allow heat to transfer and thermal equilibrium to be reached. Upon reaching equilibrium the mass inside the tank can be vented off and the process repeated until the tank walls are cooled to a sufficiently low temperature.

Chato [4] provides a concise description of the cryogenic fluid resupply process and the requirements of the main system components.

Fluid systems for resupply can be subdivided into three components, the supply tank, the transfer line, and the receiver tank. The supply tank must be emptied of liquid without ingesting vapor and maintain a level of pressure sufficient to accomplish the transfer quickly. The transfer line must connect the two tanks with a minimum of fluid loss, be conditioned to the required operating parameters, and maintain a low pressure drop. Hardware challenges include reliable docking mechanisms; transfer line disconnects capable of sealing against the vacuum of space and low heat leak transfer systems. The filling of receiver tanks in low gravity poses the most technical challenges. Fluid management challenges include the uncertainty of liquid and vapor distributions in a tank in low gravity, the need to keep maximum tank pressure low to reduce tank mass, and for cryogenic liquids the large rate of generation of vapor from the residual energy stored in tank walls. During a normal gravity fill, a top vent is kept open to vent the vapor generated during the fill process, thereby maintaining a low tank pressure. If the same approach is used in low gravity, the ullage gas may never vent. Instead of venting vapor, large amounts of liquid may be dumped 
overboard. If liquid is vented from one side of a non-propulsive vent and vapor from the other side, the spacecraft may tumble out of control. The spacecraft can be placed in an artificial gravity field by continuous thruster firing to position the ullage at a vent opening, but this may require dedicated thrusters and additional propellant. For the fill operation, maintaining control between the tanker spacecraft and the spacecraft being refueled while thrusting may be difficult due to potentially large shifts in the center of gravity. In places, such as a depot based at a space station, thrusting may be impractical due to large system size.

\subsubsection{Multi-layer Insulation (MLI)}

The threat of heat leak is an inherent problem when handling and storing cryogenic propellants in a depot architecture. Johnson in [5] details the development and advantages of multilayer insulation (MLI) systems implemented in high vacuum environments (less than $\left.4 \cdot 10^{-4} k P a\right)$. MLI is used to reduce heat leak while permitting the depot to remain light and compact. It is composed of many layers of material with a spacer material separating the layers. MLI is intended to reduce heat leak from conduction, convection, and radiation and preserve the tank contents in a near constant thermodynamic state and is a critical component for long duration storage of cold fluids in a space environment.

\subsubsection{Liquid Acquisition Devices (LADS)}

LADS leverage surface tension forces to deliver single phase liquid from the depot. The ability of extracting single phase liquid from both the supply tank and receiver tank is critical. The low gravity environment of LEO introduces complications to an already complicated problem of preferentially transferring single phase liquid. Control over fluid quality is desirable due to the varying thermodynamic behavior of liquid versus vapor and ever changing mission needs. Propellant systems require delivery of single phase liquid to op- 
erate properly.

Liquid is expected to adhere to the tank walls. LADS are positioned on the inside walls of the depot and use a fine mesh material to separate the liquid from the vapor. The higher surface tension of the liquid adheres it to the mesh and separates the liquid from the vapor. The liquid is then permitted to travel through the lad channel and out of the depot. LADS will be present in any tank required to deliver single phase liquid in a low gravity environment. The additional thermal mass of these devices must be considered when transferring relatively cold fluids, like cryogens.
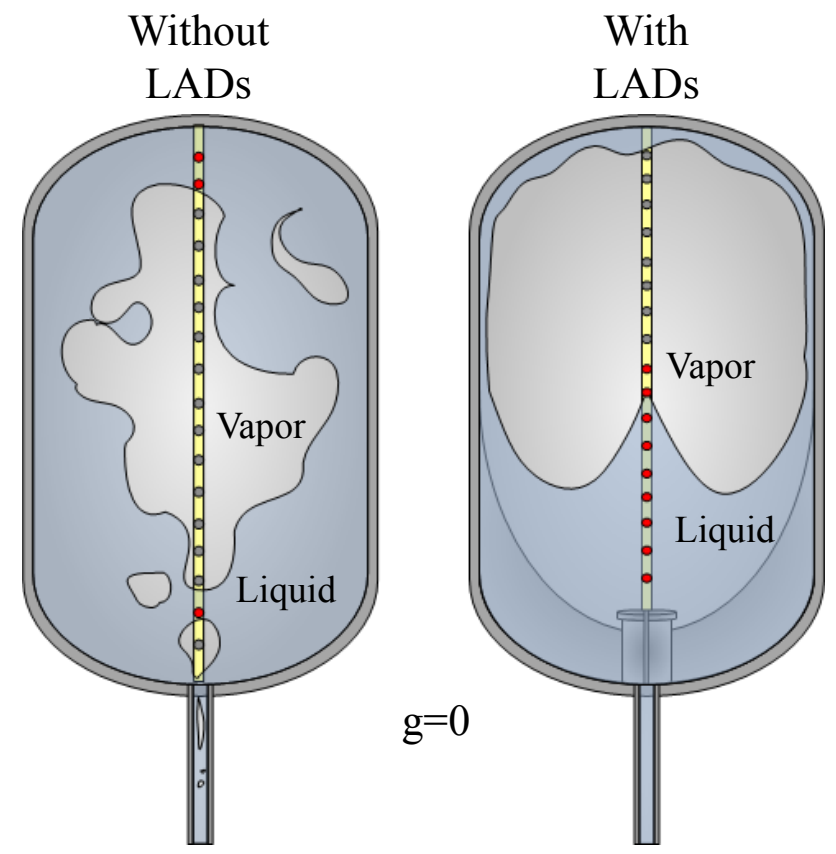

Figure 1.3: The influence of LADs on fluid position and liquid delivery. Image courtesy of [6].

\subsubsection{Transfer Line}

The transfer line possess a small thermal mass compared to the receiver tank but its behavior still must be accounted for when attempting to transfer liquid. The boiling characteris- 
tics in low gravity ensure the transfer line chilldown process is non-trivial. An actual flight scenario would require the supply liquid to be subcooled to suppress boiling in the transfer lines. Representative transfer lines are therefore included in ground based experimental setups.

\subsubsection{Spray System}

The spray system of the receiver must perform two roles. First it must deliver liquid charges to the tank during Charge-Hold-Vent (CHV). It is desirable to impinge the liquid charges onto the tank walls in the form of a jet or droplets. Droplets and jets inherently provide high rates of heat transfer with the impinged upon surface. The second role of the spray system is fulfilled during a No-Vent Fill (NVF). The spray system must encourage the vapor phase to condense into the bulk liquid phase.

Controlling the heat transfer between the fluid and tank wall is important but heat transfer between the liquid phase and vapor phase inside the tank must also be handled. When refilling a tank with liquid it is critical to condense the vapor. A well designed spray system inside the receiving tank will take the liquid fuel supplied from the depot and leverage its thermodynamic properties to absorb heat from the vapor and condense it into the bulk liquid. Spray systems may be composed of numerous nozzle heads or a single nozzle head. Shower head arrays that breakup the incoming flow into a mist of droplets and single nozzles that preserve the incoming liquid in a jet are two options for spray systems. The droplet nozzles promote the vapor to condense on the liquid droplets. The liquid jet impinges the bulk liquid and thermally destratifies. Mixing the bulk liquid drops the liquid temperature in contact with the vapor and promotes the vapor to condense. Condensing the vapor controls the pressure inside the tank which is paramount for attaining high fill levels in receiver tanks and long duration liquid storage in depots. 


\subsection{Fluid Transfer}

The transfer process can be optimized in one of two ways. The process can be time optimized which is advantageous in the presence of large heat leaks and/or strict launch/landing windows or the process can be optimized to preserve as much fluid mass as possible.

The careful use of liquid launched into orbit aboard depots is critical for a private company to be profitable and for the government to obtain every possible kg of usable payload. Techniques for transferring the liquid from the supply depot have been explored since the 60's [7]. Over fifty years have passed but the problem remains the same.

The transfer process can be broken down into two subprocesses. First the tank must be cooled down before any liquid can be injected. Next the receiving tank must undergo a nonvented fill. Each technique is specifically designed for a low-g environment. If a similar transfer were to occur within Earth's gravity the procedure's details become familiar. A vent valve would be left open on top of the warm tank as the cold liquid is injected from the bottom. At first the liquid would rapidly transfer heat with the wall, boil, and vaporize. The generated vapor would rise to the top of the tank and exit through the open vent valve. Eventually, the tank would cool enough to allow liquid accumulation in the bottom. Liquid will continue to evaporate but at a slower rate than it did initially because less thermal energy remains in the walls of the tank. The rate of liquid entering the tank would be greater than the rate of evaporation and as a result the liquid level in the tank rises. Once the tank is brimming with liquid the vent valve can be shut and the transfer is considered complete. Heat leak into the tank will cause some of the liquid to evaporate and the pressure to rise. The vent valve can be opened to relieve the pressure without the risk of losing any liquid.

In the above example the chilldown and fill occurred simultaneously. Liquid was allowed to flow into the tank at a constant rate until the tank was considered full even though rapid evaporation occurred initially. The vapor generated during the process left the tank before achieving thermal equilibrium with the tank walls. The vapor exiting the tank still 
had cooling potential. This is not a mass optimized solution to filling a warm tank with a cold liquid.

Consider performing the same 1-g fill procedure except this time the vent valve is shut. Now as the liquid evaporates due to heat transfer with the wall the pressure inside of the tank will rise. If the tank is at a high enough temperature prior to commencing the transfer the evaporation of the first slugs of liquid may over pressurize the tank and cause it to fail. To avoid over pressurization the vent valve would need to be opened. Since the vent valve is located at the top of the tank opening the valve would allow some vapor to escape, the tank pressure to drop, and the fill to continue. By cycling the vent valve the cooling power of the cold vapor can be used to cool the tank in addition to the cooling power of the incoming liquid. If the pressure increases too much the vapor is able to be preferentially vented without the risk of losing any liquid.

The amount of vapor generated in the second example, with the vent valve closed, will be less than the amount of vapor generated during the first example, with the vent valve open. Containing the vapor phase inside the tank allows it to slowly come into thermal equilibrium with the tank wall and extract more thermal energy compared to the open vent case. In this way, less liquid is converted to vapor and ultimately lost from the tank. The high cost of launching mass into space drives the need for mass optimized transfer techniques that preserve the valuable liquid phase.

Extensive testing of depot components must first take place on Earth's surface before a successful transfer can be expected to take place on orbit. Testing flight hardware is expensive and time consuming due to the hazardous nature of pressurized cryogenic fluids and the necessary safety precautions that must be adhered to. Modeling the basic thermodynamic behavior of these systems using simple and flexible computer programs helps to decrease cost and enables the design engineers to make quick decisions regarding flight hardware. 


\subsection{Literature Review}

\subsubsection{Charge-Hold-Vent (CHV)}

In [8] Chato and Sanabria experimentally investigate the feasibility of the charge-hold vent (CHV) cryogenic tank chilldown technique using liquid hydrogen and a $4.96 \mathrm{~m}^{3}$ Aluminum 2219 tank. The tank was able to be cooled from an initial wall temperature of $244 K$ in six CHV cycles. Total mass consumed during the chilldown was estimated at $14.51 \mathrm{~kg}$. A NVF was then performed and completed successfully.

Defelice and Aydelott developed an analytical thermodynamic equilibrium model of the CHV process in [9]. The code predicts equilibrium tank wall temperature and tank pressure given initial receiver tank temperature, max receiver tank pressure, supply tank saturation pressure, pressure drop value for the vent stage, receiver tank volume, and receiver tank mass. The code calculates the amount of mass injected for each cycle from an ideal gas assumption. A final approximation for the mass consumed is among the outputted values.

In [10] Honkonen, Bennett, and Hepworth developed and validated a computer code (GDNVF) capable of predicting CHV and NVF characteristics. The code implemented a droplet model to capture the heat transfer between the ullage and injected liquid. Free convection correlations model heat transfer during the hold phase of the CHV cycle. The vent phase is modeled as isentropic with additional cooling being made available with each successive vent. The code can be made to predict low gravity chilldown scenarios but validation for this aspect of the program could not be made due to a lack of low gravity experimental chilldown data. No comparison is made to 1-g chilldown data but a validation for the NVF component of GDNVF is shown. GDNVF over predicts the initial pressure rise for the liquid hydrogen NVF experiments of [11]. A version of the GDNVF code is included in the Cryogenic On-Orbit Liquid Analysis Tool (COOLANT) developed for NASA Marshall Space Flight Center.

In [12] Honkonen, Pietrzyk, and Schuster investigate the effects of persisting fluid mo- 
tion induced by pulsed injection during a general $\mathrm{CHV}$ procedure. During the hold phase of the $\mathrm{CHV}$ procedure in low gravity the dominant mode of heat transfer is due to persisting fluid motion induced by the initial injection of fluid into the tank. They found the pulsed injection technique decreased the time of chilling down a tank from a given temperature but did not offer significant savings in fluid mass. Roughly the same amount of fluid mass is needed to chilldown a tank by pulsed injection compared to normal injection techniques.

[12] used analytical models developed in [13] to describe the heat transfer behavior of the moving fluid in a low gravity environment. [13] explores two bounding methods of injection, tangential and radial. Tangential injection induces an organized flow field within the tank while radial injection impinges the tank walls which causes the associated flow field to decay quicker than the flow field associated with tangentially injected fluid. Tangential injection was found to persist six times longer than the flow field induced by radial injection which persists the forced convection mode of heat transfer and transfers more heat in less time compared to radial injection. Under low gravity conditions the chilldown time associated with tangentially injected fluid is at least three times less than the chilldown time associated with radially injected fluid. As the g-level increased the effects of radial versus tangential injection collapsed onto the same behavior due to the elevation of natural convection as the governing mode of heat transfer.

\subsubsection{No-Vent Fill (NVF)}

There has been deeper analytical investigation into the NVF procedure compared to CHV.

[14], [11], and [15] analyze the NVF procedure under normal gravity conditions but in a space like environment. A space like environment is simulated using a shadow shield, vacuum chamber, and coldguard to thermally ground conduction heat transfer along transfer lines. These tests were conducted in support of a various flight demos that never came to fruition. It was shown that NVF is a reliable technique for filling a sealed container with cryogenic fluid in a space like environment. 
In [11] Chato, Moran, and Nyland successfully fill a $0.14 \mathrm{~m}^{3}$ stainless steel tank with liquid nitrogen and liquid hydrogen, respectively. The test was conducted in Cell 7 of the Cryogenic Components Laboratory at NASA Lewis Research Center. The test was intended provide an understanding of the basic physics and driving forces of a NVF transfer despite the inability to simulate a low gravity environment. The test was conducted over a range of inlet mass flow rates, starting wall temperatures, and with a $120^{\circ}$ cone angle spray nozzle acting as the spray system. Fill levels in excess of $86 \%$ were achieved for both the hydrogen and nitrogen tests with two of the nitrogen tests reaching the $97 \%$ fill mark. Hydrogen proved difficult to use in the NVF process with one test only reaching the $14 \%$ fill level. Two analytical NVF models were then validated with the data acquired from this experiment. The experiment and model were found to not agree well. The initial pressure rise for the nitrogen tests was over predicted while the initial pressure rise for the hydrogen tests were under predicted.

In [15] Chato analyzes a liquid hydrogen NVF with a $2.01 \mathrm{~m}^{3}$ Aluminum 2219 test tank. Tests were conducted with inlet pressures of $35 k P a, 103 k P a$, and $172 k P a$, transfer pressures of $138 \mathrm{kPa}, 206 \mathrm{kPa}$, and $310 \mathrm{kPa}$, and starting wall temperatures in the range of $16 \mathrm{~K}$ to $129 \mathrm{~K}$. All tests completed with fill levels in excess of $90 \%$ except for the test at the highest wall temperature of $129 \mathrm{~K}$. A thermodynamic equilibrium model was then compared to the data with good agreement. The model did over predict the pressure behavior of the higher initial wall temperature tests. 


\section{Chapter 2}

\section{Methodology}

\subsection{System Description}

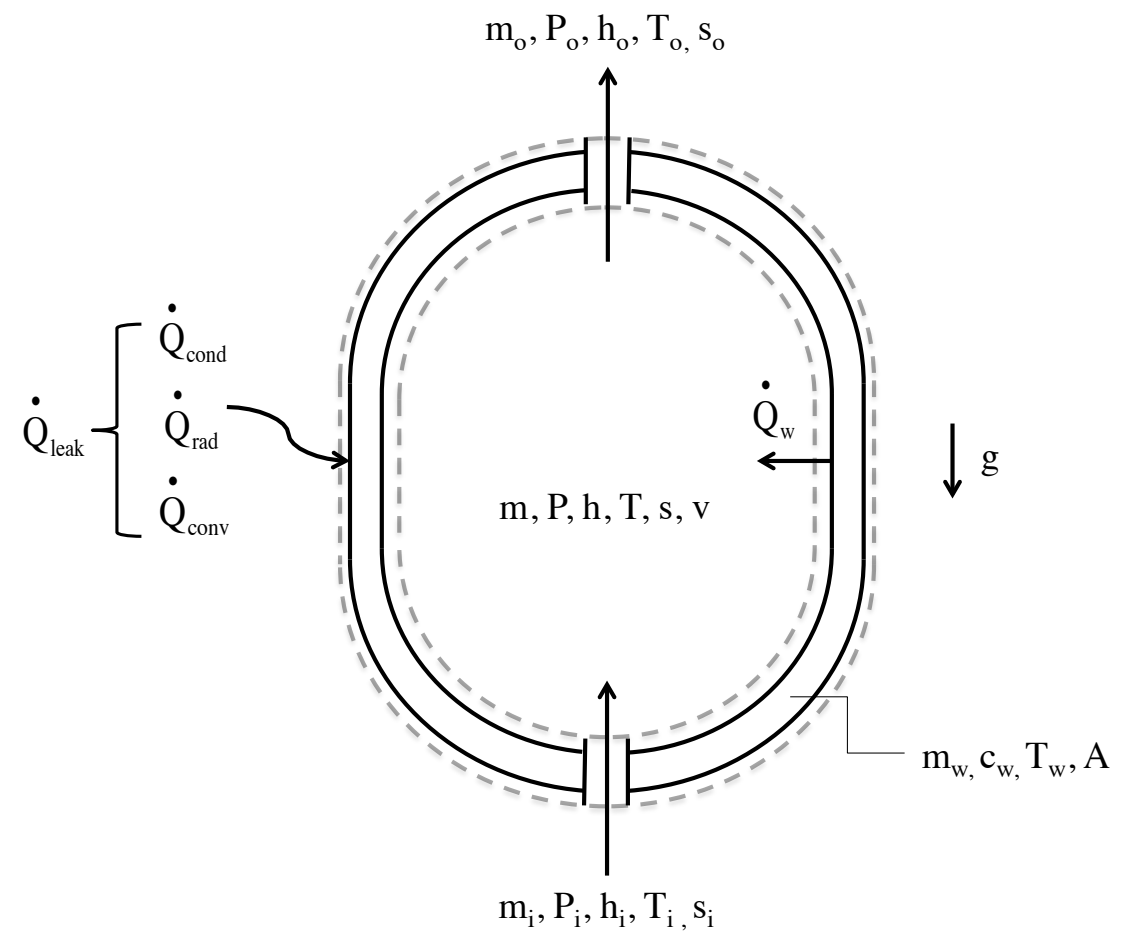

Figure 2.1: The interaction of mass and energy with the system's control volumes. 
The goal of Charge-Hold-Vent is to chilldown the walls of a container to some preselected temperature using the least amount of liquid mass possible. Preserving the liquid phase is important when dealing with space vehicle refueling. The present generation of chemical rocket engines must be delivered liquid fuel and oxidizer to operate safely.

Quantities of interest in the CHV analysis include the total mass injected over all cycles, the total time required for the chilldown to occur, and the pressure and temperature histories of the tank and tank contents. Mass injection takes place over small lengths of time relative to heat transfer. The effects of heat and mass transfer on the tank contents and tank wall control volumes are treated separately based on this analysis. Varying parameters such as tank geometry, supply pressure, inlet nozzle area, and material properties will impact the system's behavior. The relative changes in system behavior associated with altering the imposed parameters is also valuable information to the design engineer.

The CHV process is modeled as two control volumes with transient and thermodynamic behavior. The problem is broken into three separate transport phenomena, mass addition, heat transfer and mass removal. A finite amount of mass is instantaneously injected, heat is added as time increases, and a finite amount of mass is instantaneously removed once a user defined criteria is met. Figure 2.1 details the control volume interactions during one CHV cycle. Multiple cycles occur during the chilldown process with each successive cycle further cooling the tank material.

Two control volumes contain the tank wall and tank contents respectively. Heat transfer between the tank wall and environment is accounted for and heat transfer between the tank wall and working fluid is also modeled. After mass injection the tank is sealed with the fluid contents inside. Heat transfer between the tank wall and fluid increases the fluid's temperature and pressure. Heat transfer between the tank wall and environment is less than the heat transfer to the fluid. The tank wall cools as a result of these conditions.

The rate of heat transfer between the fluid and tank wall continues to decrease with the decreasing temperature difference between the wall and fluid. Once it is no longer 
beneficial to continue holding the fluid inside the tank the vent valve is opened and mass is permitted to leave the tank. The vent drops the tank pressure to near vacuum and the process is repeated with the next charge of fluid. However, the tank may be partially vented. A partial vent allows the fluid remaining inside the tank to adiabatically expand and cool. This phenomena increases the temperature difference between the fluid and wall and allows additional wall cooling to occur. Vent-hold cycles continue until the tank is returned to its initial pressure (typically evacuated). By mazimizing the fluid's cooling potential the amount of mass expended to attain a specified tank wall temperature can be minimized.

\subsubsection{Mass Injection Process (Charge)}

The injection process is modeled as instantaneous and isenthalpic. The pressure and temperature of the injected fluid changes across the injection nozzle but heat is not transferred to or from the surroundings. The initial state of the tank, before injection and heat addition, is specified (typically near vacuum). The small amount of fluid existing inside the tank before mass injection is in thermal equilibrium with the tank wall. The state of the fluid is specified as $\left(T_{w}, P^{0}\right)$. The receiver tank fluid state after injection and prior to heat addition is determined by the receiver tank's specific volume and the fluid's enthalpy. The enthalpy is calculated from the enthalpy of the small amount of fluid keeping pressure in the tank before injection and the injected fluid's enthalpy. The isenthalpic assumption determines

the enthalpy of the injected mass as the supply enthalpy and the state $\left(T_{w}, P^{0}\right)$ determines the enthalpy and mass of the fluid already in the tank. The supply state is saturated liquid at a specified pressure (typically above atmospheric). Specific volume is calculated from the known amount of mass in the tank, the injected mass plus the initial, and the tank volume.

\subsubsection{Heat Transfer Process (Hold)}

Mass is added and removed from the system quickly relative to the time required for heat transfers to occur and thermal equilibrium to be reached. Two modes of convection heat 
transfer are modeled, forced convection and natural convection. Both liquid let impingement and natural convection of the vapor contents are present in during the charge phase of $\mathrm{CHV}$. The jet impingement stirs the tank contents and impacts the wall but eventually decays to zero leaving only the natural convection of the vapor to continue transferring heat. The temperature difference between the fluid and wall, $\Delta T$, determines when the hold phase ends and venting occurs. A pressure limit, typically the receiver tank's maximum allowable working pressure (MAWP), is also set and will trigger the venting phase regardless of $\Delta T$ 's value.

\subsubsection{Vent Process}

The venting process is modeled to occur instantaneously and isentropically. The super heated vapor will cool as mass is removed from the tank making available additional cooling potential to further decrease the wall temperature. Another hold phase can follow a vent allowing time for heat to transfer between the wall and fluid remaining in the tank. The tank contents will once again increase in temperature and pressure. Once $\Delta T$ is met, the tank is vented again and the vent-hold procedure is repeated. The vapor mass remaining in the tank will eventually become small and its capacity for absorbing thermal energy from the wall is no longer significant. At this point the tank is vented to its initial pressure and another charge of mass may be injected into the tank, beginning another cycle of the CHV process.

The vent-hold strategy allows more heat to be extracted from the wall at the cost of increased time for the CHV cycle. Heat leak into the tank must be considered. The decreasing temperature of the tank and fluid system increases the potential for heat leak. The

heat transferred to the fluid during the hold must be greater than the heat transfer from the ambient for the vent-hold process to be beneficial. 


\subsubsection{Receiver Tank}

\subsubsection{Material Properties}

The tank is modeled as a single temperature node with a single mass and uniform material properties. The tank wall's state is specified by the properties $T_{w}$ and $C_{w}$. Material property models are available in functional form and files of tabulated property data are also used. The functional property models are of the form:

$$
\begin{aligned}
\log _{10} C_{w}\left(T_{w}\right)= & a+b\left(\log _{10} T_{w}\right)+c\left(\log _{10} T_{w}\right)^{2}+d\left(\log _{10} T_{w}\right)^{3}+e\left(\log _{10} T_{w}\right)^{4}+ \\
& f\left(\log _{10} T_{w}\right)^{5}+g\left(\log _{10} T_{w}\right)^{6}+h\left(\log _{10} T_{w}\right)^{7}+i\left(\log _{10} T_{w}\right)^{8}
\end{aligned}
$$

Where $C_{w}$ is the tank wall specific heat, $T_{w}$ is the tank wall temperature, and the coefficients $a-i$ are specified for each material. Eqn (2-1) is used to calculate the material specific heat and is valid in the temperature range $4 K-300 K$.

Data sets from [16] are interpolated to provide property values for a wider range of temperatures compared to the functional models. Not all data points in the tables were found experimentally. Data points below $100 \mathrm{~K}$ were calculated using the Kopp-Newman Law, Eqn (2-2). The Kopp-Neumann Law states the specific heat for alloys can be calculated from the total number of alloy constituents, $N$, the specific heat, $C_{i}$, of each constituent, and the mass fraction, $f_{i}$, of each constituent.

$$
C=\sum_{i=1}^{N}\left(C_{i} \cdot f_{i}\right)
$$

\subsubsection{Geometry}

The code models spherical tanks and any combination of hemisphere, oblate hemispheroid, and prolate hemispheroid endcaps separated by a cylindrical tank body. The tank segments impose geometric characteristics onto the heat transfer behavior through the differing area

and volume relationships inherent in the different geometries. These details are handled by 
the heat transfer model. The differing area relationships impact the wall area available for heat transfer and the differing volumes change the specific volume of the fluid.

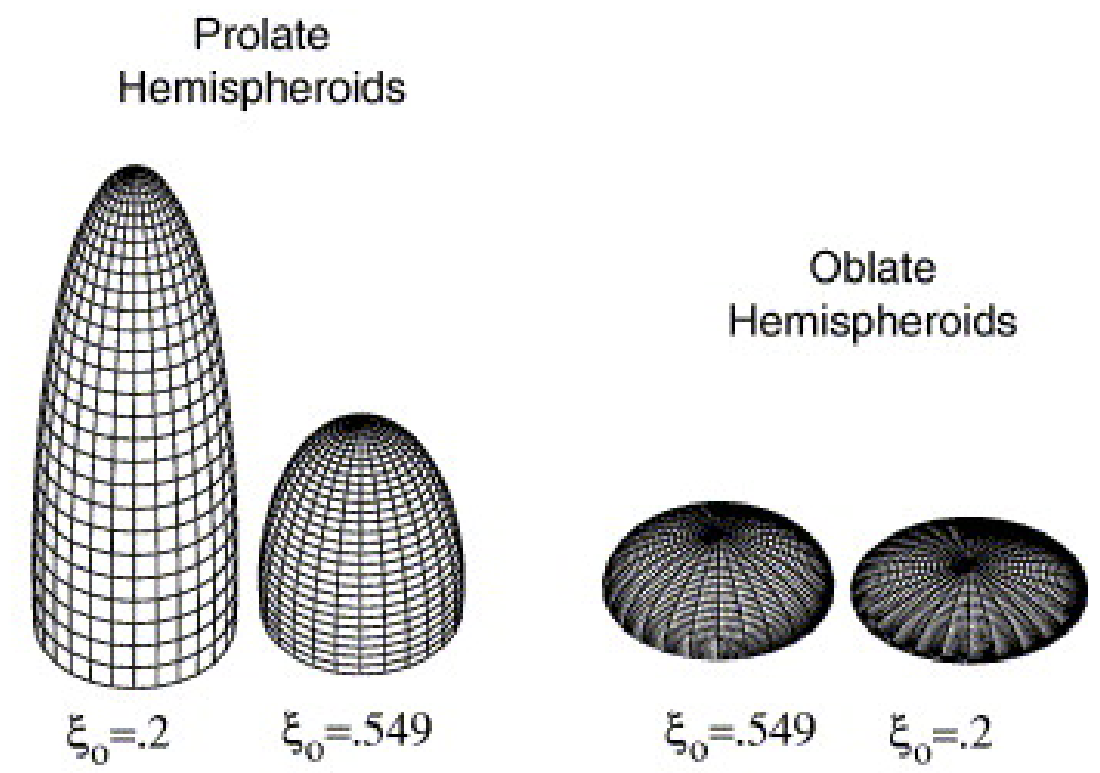

Figure 2.2: Examples of oblate and prolate hemispheroids at two different height to width ratios. $\xi_{0}=0.549$ corresponds to $h / w=2$ (prolate) and $h / w=1 / 2$ (oblate). Image courtesy of [17].

\subsubsection{Fluid}

The fluid is modeled as a single temperature and pressure node and properties are uniform throughout. Boundary layer effects, temperature gradients, and two phase boiling behavior are not accounted for. The fluid state is specified by $m, P, h, T, s, v$ in Fig 2.1.

Fluid properties are obtained via a set of fortran subroutines produced by NIST. The subroutines are collectively named REFPROP. The introductory paragraphs to the REFPROP user's manual summarize the program's capabilities [18].

REFPROP is an acronym for REFerence fluid PROPerties. This program, developed by the National Institute of Standards and Technology (NIST), provides tables and plots of the thermodynamic and transport properties of indus- 
trially important fluids and their mixtures with an emphasis on refrigerants and hydrocarbons, especially natural gas systems.

REFPROP is based on the most accurate pure fluid and mixture models currently available. It implements three models for the thermodynamic properties of pure fluids: equations of state explicit in Helmholtz energy, the modified Benedict-Webb-Rubin equation of state, and an extended corresponding states (ECS) model. Mixture calculations employ a model that applies mixing rules to the Helmholtz energy of the mixture components; it uses a departure function to account for the departure from ideal mixing. Viscosity and thermal conductivity are modeled with either fluid-specific correlations, an ECS method, or in some cases the friction theory method.

These models are implemented in a suite of FORTRAN subroutines. They are written in a structured format, are internally documented with extensive comments, and have been tested on a variety of compilers. Routines are provided to calculate thermodynamic and transport properties at a given $(\mathrm{T}, \rho, \mathrm{x})$ state. Iterative routines provide saturation properties for a specified $(\mathrm{T}, \mathrm{x})$ or $(\mathrm{P}, \mathrm{x})$ state. Flash calculations describe single- or two-phase states given a wide variety of input combinations $[(\mathrm{P}, \mathrm{h}, \mathrm{x}),(\mathrm{P}, \mathrm{T}, \mathrm{x})$, etc $]$.

The following properties can be calculated using REFPROP. Unit conversion is handled external to the REFPROP subroutines. All property calls must conform to the units listed below. 


\begin{tabular}{cll} 
Symbol & Property & Units \\
\hline$T$ & temperature & $\mathrm{K}$ \\
$P$ & pressure & $\mathrm{kPa}$ \\
$\rho$ & density & $\mathrm{mol} / \mathrm{L}$ \\
$z$ & composition & mole fraction \\
$x$ & quality & mole basis (moles vapor/total moles) \\
$h, u$ & enthalpy, internal energy & $\mathrm{J} / \mathrm{mol}$ \\
$g, f$ & Gibbs, Helmholtz free energy & $\mathrm{J} / \mathrm{mol}$ \\
$s, c$ & entropy, heat capacity & $\mathrm{J} /(\mathrm{mol} . \mathrm{K})$ \\
$a$ & speed of sound & $\mathrm{m} / \mathrm{s}$ \\
$\mu_{j t}$ & Joule-Thompson coefficient & $\mathrm{K} / \mathrm{kPa}$ \\
$\mu$ & viscosity & $\mu \mathrm{Pa} . \mathrm{s}\left(10^{-6} \mathrm{~Pa} . \mathrm{s}\right)$ \\
$k$ & thermal conductivity & $\mathrm{W} /(\mathrm{m} \cdot \mathrm{K})$ \\
$\tau$ & surface tension & $\mathrm{N} / \mathrm{m}$
\end{tabular}

Table 2.1: Thermodynamic Properties available through REFPROP.

\subsection{Development of Equations}

\subsubsection{Control Volume Analysis}

The tank wall and tank fluid contents are each wholly contained in separate control volumes. Structuring the analysis in this manner allows heat and work interactions between the two control volumes to be monitored as functions of time based on the time dependent governing equations of the respective control volumes.

\subsubsection{Continuity: Charge}

The mass flow rate entering the tank is assumed constant over the time of injection. Changes in pressure with in the receiver tank and the effects it has on flow rate are neglected. Solving for the duration of mass injection is accomplished by specifying the amount of mass 
desired to be injected and the flow rate at which the mass is injected.

$$
\begin{gathered}
\frac{\mathrm{d} m}{\mathrm{~d} t}=\dot{m}_{i} \\
\frac{\mathrm{d} m}{\dot{m}_{i}}=\mathrm{d} t \\
\int_{0}^{m_{i}} \frac{\mathrm{d} m}{\dot{m}_{i}}=\int_{0}^{t_{i}} \mathrm{~d} t \\
t_{i}=\frac{m_{i}}{\dot{m}_{i}}
\end{gathered}
$$

Where $m$ is the mass of fluid inside the tank, $m_{i}$ is the amount of mass injected into the tank for the current $\mathrm{CHV}$ cycle, $\dot{m}_{i}$ is the average mass flow rate of injection, $\Delta t$ is the time step size, and $t_{i}$ is the duration of the injection. $t_{i}$ is used in the heat transfer calculations to establish regimes of jet impingement heat transfer. $m_{i}$ and $\dot{m}_{i}$ are specific to tank hardware and are input parameters.

\subsubsection{Nozzle Velocity}

Nozzle velocity is calculated from the Bernoulli equation shown in Eqn (2-7) and Eqn (2-8). The jet velocity decreases with increasing tank pressure and captures the decaying heat transfer characteristics of the jet. The jet velocity is used in calculation of the associated jet Reynolds number which is used in calculation of the jet Nusselt number and jet impingement heat transfer coefficient. The free fluid surface velocity inside the supply tank is relatively small compared to the velocity at the nozzle exit. It is neglected in the analysis.

$$
\begin{gathered}
P_{s}+1 / 2 \rho_{s} V_{s}^{2^{0}}=P+1 / 2 \rho_{s} V_{j e t}^{2} \\
V_{j e t}=C_{D} \sqrt{\frac{2\left(P_{s}-P\right)}{\rho_{s}}}
\end{gathered}
$$


Where $C_{D}$ is the nozzle discharge coefficient, $P_{s}$ is the supply pressure, $V_{s}$ is the velocity of the free fluid surface inside the supply tank, $\rho_{s}$ is the supply fluid density, $P$ is the receiver tank pressure, and $V_{\text {jet }}$ is the jet velocity at the nozzle exit.

\subsubsection{Continuity: Vent}

The process of venting mass is modeled as pseudo-instantaneous. A single vent is divided into smaller vent-hold cycles and the mass leaving the tank changes with each passing subvent. The mass removal behavior is determined by $f(t)$ which is dependent on a constant mass scaling factor, $m_{o}^{1}$, calculated in Eqn (2-10)

$$
\begin{aligned}
m_{o, t o t} & =m_{o}^{1} \int_{0}^{t_{o}} f(t) \mathrm{d} t \\
m_{o}^{1} & =\frac{m_{o, t o t}}{\int_{0}^{t_{o}} f(t) \mathrm{d} t}
\end{aligned}
$$

Where $f(t)$ is the functional behavior of the mass removal, $m_{o, t o t}$ is the specified total mass removed from the tank during the parent vent, and $t_{o}$ is the duration of the parent vent.

Approaching the process in a discontinuous manner allows mass removal to remain instantaneous while still permitting heat to transfer pseudo-simultaneously. A timescale does not exist for mass removal because the process is modeled as instantaneous. The change in receiver tank mass as a function of time is found in Eqn (2-13).

$$
\begin{gathered}
\frac{\mathrm{d} m}{\mathrm{~d} t}=-\dot{m}_{o} \\
\frac{m^{j+1}-m^{j}}{\Delta t}=-\frac{m_{o}^{1}}{\Delta t} f(t)=-\frac{1}{\Delta t} \frac{m_{o, t o t}}{\int_{0}^{t_{o}} f(t) \mathrm{d} t} f(t) \\
m^{j+1}=m^{j}-\frac{m_{o, t o t}}{\int_{0}^{t_{o}} f(t) \mathrm{d} t} f(t)
\end{gathered}
$$




\subsubsection{Energy Balance: Fluid}

The enthalpy of the tank contents is tracked through time using an energy balance on the tank fluid contents. As time increases, heat is removed from the tank wall and added to the tanks fluid contents. $\dot{Q}_{w}$ contains all modes of heat interactions between the wall and the fluid. $h_{i}^{j}$ is specified by the user for the first time step while $h_{i}^{j+1}$ is calculated from Eqn (2-18). The calculated value is stored in $h_{i}$ for the next time step. The state of the fluid contents is assumed to be uniform and is modeled as a single thermodynamic node/control volume. Variations in fluid temperature near the zone of heat transfer with the wall are accounted for in the heat transfer model. Mass addition and subtraction to the fluid control volume is treated as instantaneous and allows the fluid mass inside the tank to remain constant during time stepping. Thus $m$ is moved outside the differential and a finite difference approximation is used to calculate fluid specific enthalpy as a function of time.

$$
\begin{gathered}
\frac{\mathrm{d} E}{\mathrm{~d} t}=\dot{Q}-\dot{W} \\
\frac{\mathrm{d} U}{\mathrm{~d} t}=\dot{Q}_{w}-\frac{\mathrm{d} P V}{\mathrm{~d} t} \\
\frac{\mathrm{d}(m u+m P v)}{\mathrm{d} t}=\dot{Q}_{w} \\
\frac{\mathrm{d}(m \cdot h)}{\mathrm{d} t}=\dot{Q}_{w} \\
m^{j} \cdot \frac{h^{j+1}-h^{j}}{\Delta t}=\dot{Q}_{w}^{j}
\end{gathered}
$$

Where $E$ is the total energy of the fluid, $\dot{Q}$ is the net rate of heat transfer with the fluid's control volume, $\dot{W}$ is the net rate of work transfer with the control volume, $U$ is the internal energy of the fluid, $u$ is the specific internal energy of the fluid, $\dot{Q}_{w}^{j}$ is the rate of heat transfer between all sections of the tank wall and the fluid, $P$ is the pressure of the fluid, $V$ is the volume occupied by the fluid, $v$ is the specific volume of the fluid, $h$ is the specific 
enthalpy of the fluid, $h^{j+1}$ is the specific enthalpy of the fluid after one time step of heat addition, and $h^{j}$ is the specific enthalpy of the fluid before a time step of heat addition. $m_{j}$ and $\dot{Q}_{w}^{j}$ may change with each time step but do not change across time steps.

\subsubsection{Energy Balance: Tank Wall}

An energy balance on the tank wall calculates the wall temperature at each time step. $C_{w}$ is a function of $T_{w}$. The time steps are specified to be small and $C_{w}$ does not experience significant change across these small instances of time. $C_{w}$ is moved outside the differential and a finite difference approximation is applied to the tank wall temperature derivative. The tank wall is modeled as a single and uniform temperature, temperature gradients across the thickness and between neighboring sections of tank wall are not considered. Tank thickness is typically small and insulation helps protect the external tank wall from ambient heat leak. For example, assuming a spherical tank geometry $\frac{\partial T_{w}}{\partial r}=\frac{\partial T_{w}}{\partial \theta}=\frac{\partial T_{w}}{\partial \phi} \approx 0$. The wall also does not experience significant work interactions.

$$
\begin{gathered}
\frac{\mathrm{d} E_{w}}{\mathrm{~d} t}=\dot{Q}-\dot{W}^{0} \\
\frac{\mathrm{d} U_{w}}{\mathrm{~d} t}=\left(\dot{Q}_{\text {leak }}-\dot{Q}_{w}\right) \\
\frac{\mathrm{d}\left(m_{w} C_{w} T_{w}\right)}{\mathrm{d} t}=\left(\dot{Q}_{\text {leak }}-\dot{Q}_{w}\right) \\
\left(m_{w} C_{w}\right) \cdot \frac{\mathrm{d} T}{\mathrm{~d} t}=\left(\dot{Q}_{\text {leak }}-\dot{Q}_{w}\right) \\
\left(m_{w} C_{w}^{j}\right) \cdot \frac{T_{w}^{j+1}-T_{w}^{j}}{\Delta t}=\left(\dot{Q}_{\text {leak }}^{j}-\dot{Q}_{w}^{j}\right)
\end{gathered}
$$

Where $E_{w}$ is the total energy of the tank wall, $U_{w}$ is the internal energy of the tank wall, $\dot{Q}_{\text {leak }}$ is all modes of heat transfer between the tank wall and the environment, $m_{w}$ is the total tank mass, $T_{w}^{j+1}$ is the tank wall temperature after one time step of heat exchange, $T_{w}^{j}$ is the tank wall temperature before the time step of heat exchange. $\dot{Q}_{\text {leak }}^{j}$ and $C_{w}^{j}$ may 
change with each time step but are constant across the time step.

\subsubsection{Heat Interaction Breakdown}

The heat transfer between the wall and fluid is composed of four components. The initial injection of mass into the tank contributes an impinging jet heat transfer mode, $Q_{j e t}$. The presence of cold vapor, a warm wall, and a gravitational field contribute free convection modes of heat transfer in all sectors of the tank, $\dot{Q}_{t o p}, \dot{Q}_{c y l}$, and $\dot{Q}_{b o t}$. During the early stages of the chilldown cycle all four modes are present. Once the jet stops injecting fluid, only the free convection modes continue to transfer heat. Free convection is only considered for the vapor phase of the fluid.

An average film temperature between the wall and impinging liquid is used in the jet heat transfer expression Eqn (2-25). Jet properties are evaluated using the supply pressure and assuming a saturated state at the jet nozzle. Natural convection fluid properties are evaluated using a film temperature between the bulk fluid at temperature $T$ and the wall at temperature $T_{w}$. The natural convection heat transfer driving temperature is left as the bulk fluid temperature, $T$, and not modeled as the film temperature.

$$
\begin{gathered}
\dot{Q}_{w}=\dot{Q}_{j e t}+\dot{Q}_{t o p}+\dot{Q}_{c y l}+\dot{Q}_{b o t} \\
\dot{Q}_{j e t}=h_{j e t} A_{j e t}\left(T_{w}-T_{j e t}\right)=h_{j e t}^{j} A_{j e t}\left(T_{w}^{j}-T_{j e t}^{j}\right) \\
\dot{Q}_{t o p}=h_{t o p} A_{t o p}\left(T_{w}-T\right)=h_{t o p}^{j} A_{t o p}\left(T_{w}^{j}-T^{j}\right) \\
\dot{Q}_{c y l}=h_{c y l} A_{c y l}\left(T_{w}-T\right)=h_{c y l}^{j} A_{c y l}\left(T_{w}^{j}-T^{j}\right) \\
\dot{Q}_{b o t}=h_{b o t} A_{b o t}\left(T_{w}-T\right)=h_{b o t}^{j} A_{b o t}\left(T_{w}^{j}-T^{j}\right) \\
T_{j e t}=\frac{T_{s}+T_{w}}{2}=\frac{T_{s}^{j}+T_{w}^{j}}{2}
\end{gathered}
$$


Where $\dot{Q}_{t o p}$ is the rate of heat transfer due to natural convection between the top endcap and the vapor, $\dot{Q}_{c y l}$ is the rate of heat transfer due to natural convection between the cylindrical tank body and the vapor, $\dot{Q}_{b o t}$ is the rate of heat transfer due to natural convection between the bottom endcap and the vapor, $h_{j e t}$ is the jet heat transfer coefficient, $h_{t o p}$ is the natural convection heat transfer coefficient between the top endcap and the vapor, $h_{c y l}$ is the natural convection heat transfer coefficient between the cylindrical tank body and the vapor, $h_{\text {bot }}$ is the natural convection heat transfer coefficient between the bottom endcap and the vapor, $T_{j e t}$ is the approximate temperature of the jet, $T_{s}$ is the supply fluid temperature. The supply conditions do not change with time during a CHV cycle but can be set to different values for each cycle. The supply conditions are specified independent of the chilldown process.

The first term on the right of Eqn (2-31) represent heat transfer through the MLI system due to conduction, the second term represents heat transfer due to radiation, and the last term represents gaseous conduction heat transfer due to the presence of gas within the insulation. The gaseous conduction term may be neglected for tanks in near vacuum environments where the pressure between the layers of insulation is assumed to be equivalent to the ambient pressure. Eqn (2-31) is the physics based heat leak equation through an MLI system and Eqn (2-32) is the modified heat leak equation based on empirical data.

$$
\begin{gathered}
\dot{Q}_{\text {leak }}=\dot{Q}_{\text {cond }}+\dot{Q}_{\text {rad }}+\dot{Q}_{\text {conv }} \\
=C_{2} f k \frac{\left(T_{a}-T_{w}\right)}{\Delta x}+\frac{\sigma\left(T_{a}^{4}-T_{w}^{4}\right)}{\left(\frac{1}{\epsilon_{h}}+\frac{1}{\epsilon_{c}}-1\right)}+C_{1} P_{a} \alpha\left(T_{a}-T_{w}\right) \\
\dot{Q}_{\text {leak }}=\frac{C_{S} \bar{N}^{2.56}}{N_{S}} \cdot \frac{T_{a}^{2}-T_{w}^{2}}{2}+\frac{C_{R} \epsilon}{N_{S}}\left(T_{a}^{4.67}-T_{w}^{4.67}\right)+\frac{C_{P} P_{a}^{\sigma^{0}}}{N_{S}}\left(T_{a}^{0.26}-T_{w}^{0.26}\right)
\end{gathered}
$$

Where $C_{s}$ is an empirical coefficient of solid conduction, $C_{R}$ is an empirical coefficient of radiation heat transfer, $\epsilon$ is the insulation sheet emissivity, $\bar{N}$ is the insulation layer density, $N_{s}$ is the number of insulation sheets, $T_{a}$ is the ambient temperature (also specified as the 
temperature of the outer most MLI layer), $P_{a}$ is the ambient pressure, and $C_{p}$ is the specific heat at constant pressure of the gas trapped between the layers of insulation.

During the $\mathrm{CHV}$ process the heat leak is small compared to the heat transfer between

the fluid and tank components. $\dot{Q}_{l e a k}$ is typically on the order of Watts where heat transfer interactions between the wall and fluid are on the order of kilowatts.

\subsection{Method of Solution}

The thermodynamics are posed by a system of time dependent coupled equations. Two numerical treatments are implemented to solve the set and obtain approximations for the system's transient behavior. First, Lower-Upper Decomposition solves for the matrix of correction values necessary to step the system towards convergence. Using the governing equations and their derivatives, both evaluated at initial guesses to the solution, LU Decomposition calculates the correction matrix. Next, a Multi-Dimensional Newton-Raphson Iteration applies these corrections to each of the initial guesses and steps the system towards convergence. The process is repeated within a single timestep until convergence criteria is met. Only then is the solution moved forward in time.

Equations (2-38) to (2-45) mathematically detail the process using matrix notation. Governing equation partial derivatives are calculated numerically by a user defined perturbation and initial guesses at the governing variables. The coupled nature of the govering equations necessitates their simultaneous solution.

A multi-variable system must caculate $\Delta x$ for each of the unknowns at each step of the method. LU-Decomposition handles the calculation of each $\Delta x$. 


\subsubsection{Multi-Variable Newton-Raphson Iteration Method}

The $n$ governing equations must first be written as left-hand-side equal to zero.

$$
\begin{gathered}
f_{1}\left(x_{1}, x_{2}, x_{3}, \ldots x_{n}\right)=0 \\
f_{2}\left(x_{1}, x_{2}, x_{3}, \ldots x_{n}\right)=0 \\
\ldots \\
f_{n}\left(x_{1}, x_{2}, x_{3}, \ldots x_{n}\right)=0
\end{gathered}
$$

The $f_{i}\left(x_{j}\right)$ equations are then evaluated at the user's initial guesses at the $x_{j}$ variables. This is considered the zeroeth iteration of the solver. The results of each function evaluation are compared against the user's specified convergence criteria, $\epsilon$.

$$
\left|f_{i}\left(x_{j}\right)\right|<\epsilon, i=1,2, \ldots n
$$

If convergence is not met by every function then partial derivatives of each $f_{i}\left(x_{j}\right)$ are numerically calculated and evaluated with the initial $x_{j}$ guesses. $\delta$ is a user specified small perturbation necessary for numerical calculation of the first derivatives. The derivatives are stored in $[A]$ and the LU Decomposition process to calculate $[\Delta x]$, the matrix of correcting values, begins.

Analogous components exist between the multi-variable Newton-Raphson method and the more familiar single-variable method. The derivative of the single function, $\frac{\partial f}{\partial x}$, is replaced with, $[A]$, a matrix of partial derivatives. The single function, $f(x)$, is replaced with, $[f]$ a vector of multivariable functions. The expected change, $\Delta x$, in the single-variable solution, $x$, is replaced with a vector of correction terms, $[\Delta x]$, for the multi-variable solution

vector, $[x]$. Equations (2-38) to (2-45) illustrate the LU-Decomposition approach to solve for the matrix of correction values, $[\Delta x]$. The LU-Decomposition treatment is posed using matrix notation for brevity. 
It is assumed that $[A]$ may be decomposed into the lower and upper triangular matrices, $[L]$ and $[U]$. This process is shown in Eqn (2-42) with Eqn (2-49) and (2-50) containing the details of calculating the specific elements of $[L]$ and $[U]$. The $[y]$ matrix is found by forward substitution in Eqn $(2-44)$ and finally the $[\Delta x]$ is found by backward substitution in Eqn (2-45). Once again, each $x_{j}$ variable has an associated $\Delta x_{j}$ that is stored in $[\Delta x]$. Once $[\Delta x]$ is found the Multi-Variable Newton Raphson iterative technique, shown in Eqn (2-46), is used to step the set of $n$ variables towards convergence. The state of convergence is checked with Eqn (2-36) and the process repeats from Eqn (2-37) if convergence is not satisfied.

$$
\begin{aligned}
& {\left[\frac{\partial f_{i}}{\partial x_{j}}\right] \cdot[\Delta x] \approx\left[f_{i}\right]} \\
& {[A] \cdot[\Delta x]=[f]} \\
& a_{i j}=\frac{\partial f_{i}}{\partial x_{j}}, \quad i=1,2, \ldots n, \quad j=1,2, \ldots n \\
& \delta_{j}=x_{j} \cdot \epsilon \\
& \frac{\partial f_{i}}{\partial x_{j}} \approx \frac{f_{i}\left(x_{j}+\delta_{j}\right)-f_{i}\left(x_{j}-\delta_{j}\right)}{\left(x_{j}+\delta_{j}\right)-\left(x_{j}-\delta_{j}\right)} \\
& {[A]=[L][U]} \\
& {[L]([U][\Delta x])=[f]} \\
& {[L][y]=[f]} \\
& {[U][\Delta x]=[y]} \\
& x_{j}=x_{j}-\Delta x_{j}, j=1,2, \ldots n
\end{aligned}
$$




\subsubsection{LU Decomposition}

$$
\begin{gathered}
{\left[\begin{array}{cccc}
a_{11} & a_{12} & \cdots & a_{1 n} \\
a_{21} & a_{22} & \cdots & a_{2 n} \\
\vdots & \vdots & \ddots & \vdots \\
a_{n 1} & a_{n 2} & \cdots & a_{n n}
\end{array}\right]=\left[\begin{array}{cccc}
l_{11} & 0 & \cdots & 0 \\
l_{21} & l_{22} & \cdots & 0 \\
\vdots & \vdots & \ddots & \vdots \\
l_{n 1} & l_{n 2} & \cdots & l_{n n}
\end{array}\right] \cdot\left[\begin{array}{cccc}
1 & u_{12} & \cdots & u_{1 n} \\
0 & 1 & \cdots & u_{2 n} \\
\vdots & \vdots & \ddots & \vdots \\
0 & 0 & \cdots & 1
\end{array}\right]} \\
l_{i j}=a_{i j}-\sum_{k=1}^{j-1} l_{i k} \cdot u_{k j}, j \leq i, i=1,2, \ldots n \\
u_{i j}=\frac{a_{i j}-\sum_{k=1}^{i-1} l_{i k} \cdot u_{k j}}{l_{i i}}, i \leq j, j=2,3, \ldots n \\
\text { For } i=1, u_{1 j}=\frac{a_{1 j}}{l_{11}}=\frac{a_{1 j}}{a_{11}}
\end{gathered}
$$

Forward substitution to find $[y]$ :

$$
\begin{gathered}
{[L] \cdot[y]=[f]} \\
y_{1}=\frac{f_{1}}{l_{11}} \\
y_{i}=\frac{f_{i}-\sum_{k=1}^{i-1} l_{i k} y_{k}}{l_{i i}}, i=2,3, \ldots n
\end{gathered}
$$


After finding $[y]$ backward substitute to find $[\Delta x]$ :

$$
\begin{gathered}
{[U] \cdot[\Delta x]=[y]} \\
\Delta x_{n}=y_{n} \\
\Delta x_{j}=y_{j}-\sum_{k=n}^{j+1} u_{j k} \Delta x_{k}, j=n-1, n-2, \ldots 1
\end{gathered}
$$

The system simulation's governing equations can be written to conform with the above format. Eqn (2-59) is iterated to find $h^{j+1}$ and Eqn (2-60) is iterated to find $T_{w}^{j+1}$. All other thermodynamic properties are calculated by REFPROP at the state $\left(h^{j+1}, v\right)$.

$$
\begin{gathered}
f_{1}=m^{j} \cdot \frac{h^{j+1}-h^{j}}{\Delta t}-\dot{Q}_{w}^{j}=0 \\
f_{2}=\left(m_{w} C_{w}^{j}\right) \cdot \frac{T_{w}^{j+1}-T_{w}^{j}}{\Delta t}-\left(\dot{Q}_{l e a k}^{j}-\dot{Q}_{w}^{j}\right)=0
\end{gathered}
$$

\subsection{Heat Transfer Model}

\subsubsection{Impinging Jet}

The state of the jet is specified as a saturated liquid at the supply pressure, $P_{s}$. Properties necessary for Nusselt number and Rayleigh number calculations are found at this state. Heat transfer associated with the jet is modeled to take place at the temperature difference $\left(T_{w}-T_{j e t}\right)$, where $T_{j e t}$ is calculated in Eqn (2-29). $T_{j e t}$ represents a film temperature between the jet and tank wall.

The free surface jet and the submerged jet are two fundamental classifications of jets. $R e_{j e t}$ and $P r_{j e t}$ refer to the jet Reynolds number and Prandtl number respectively. $R e_{j e t}$ is 


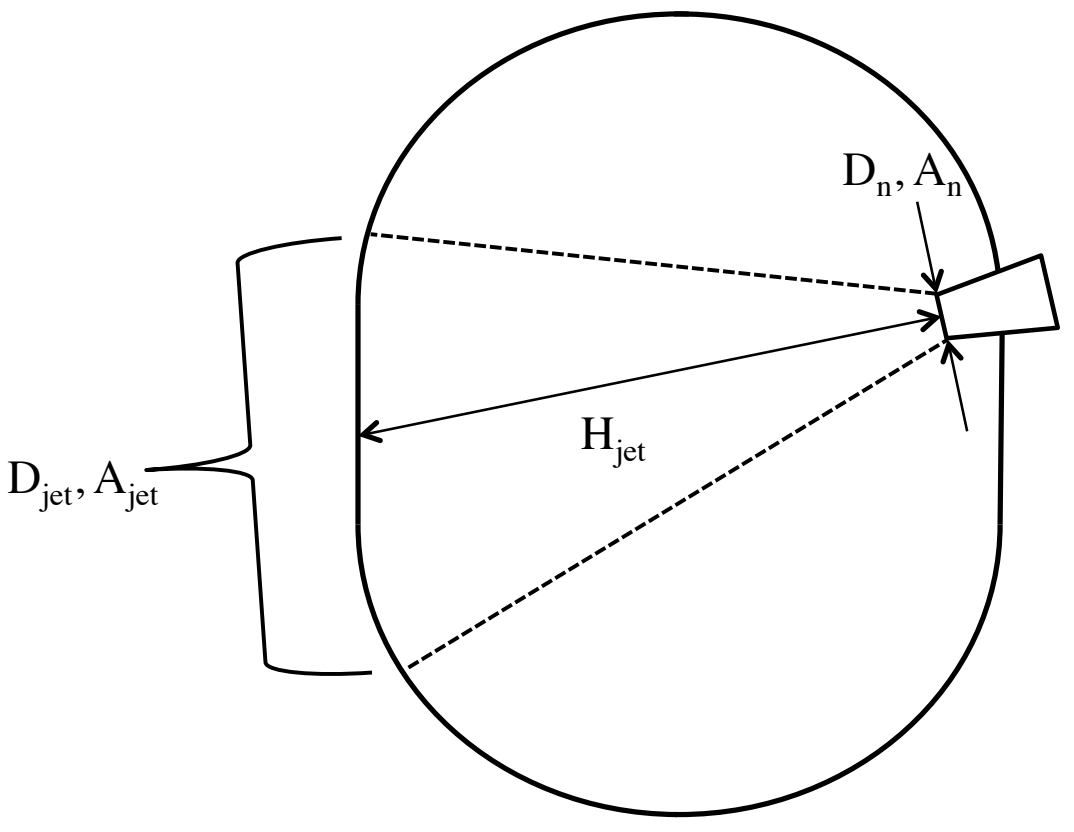

Figure 2.3: Arbitrary location and orientation of inlet nozzle. The nozzles location and orientation is specified by $H_{j e t}$ and $A_{j e t}$. The jet may be modeled at any place with in the tank impinging on any of the tanks internal surfaces.

calculated with the hydraulic diameter of the nozzle acting as the characteristic length.

$$
R e_{j e t}=\frac{\rho_{j e t} V_{j e t} D_{h}}{\mu_{j e t}}
$$

The Prandtl number is calculated as follows.

$$
P r_{j e t}=\frac{\mu_{j e t} c_{p, j e t}}{k_{j e t}}
$$

Where $\mu_{j e t}$ is the dynamic viscosity, $c_{p, j e t}$ is the specific heat at constant pressure, and $k_{j e t}$ is the thermal conductivity.

Impinging liquid jets provide high rates of heat transfer near the stagnation zone. Heat transfer rates decrease away from the stagnation zone and cause the average Nusselt number to be of moderate magnitude. This effect is more pronounced if the area requiring cooling is 
much larger than the area of the jet. The jets cause a non-uniform temperature distribution in the surface [19].

Three distinct categories of jets were considered during development. The characteristics of the turbulent jet used during data acquisition were best captured by the free liquid jet correlation provided by Jiji. However, the flexibility to switch between varying jet Nusselt number correlations allows the code to handle a wider range of chilldown approaches so all three correlations are included.

\subsubsection{Free Liquid Jet}

[20] gives a relation for an impingement cooling average Nusselt number relation for three different square heater surface configurations. The surface were impinged upon by an array of free surface water jets of diameter $0.5 \mathrm{~mm}$ and $1 \mathrm{~mm}$. Velocities of $9 \mathrm{~m} / \mathrm{s}$ and $18 \mathrm{~m} / \mathrm{s}$ and Reynolds numbers of 10000 and 20000 were considered during the tests with water. The average heat flux attained was $9.5 \cdot 10^{5} \mathrm{~W} / \mathrm{m}^{2}$. The impinging liquid water jets were perpendicular to the surfaces which were vertically oriented. Eqn (2-63) was found to represent average Nusselt number behavior for all of the three configurations.

$$
\overline{N u}_{j e t}=3.84 \cdot R e_{\text {jet }}^{1 / 2} \cdot \operatorname{Pr}_{\text {jet }}^{1 / 3} \cdot\left(\frac{0.008 \cdot n D_{j e t}}{D_{n}}+1\right)
$$

Where $D_{j e t}$ is the heater surface characteristic length, $n$ is the number of jets, and $D_{n}$ is the diameter of each jet. The cross flow between each jet is assumed to be negligible and each jet influences a single flow cell. Each jet is therefore treated as a single impinging jet, independent from the rest.

\subsubsection{Gas Jet}

[21] gives Eqn (2-64), a relation for a gas jet issuing from a single round nozzle. The jet is assumed to be turbulent and have a uniform velocity profile when exiting the nozzle. It is 
discharged into a quiescent ambient [24].

$$
\begin{gathered}
\overline{N u}_{\text {jet }}=G\left(\frac{R_{t n k}}{D_{n}}, \frac{H_{j e t}}{D_{n}}\right) \cdot F_{1}\left(R e_{j e t}\right) \\
A_{r}=\left(\frac{D_{n}}{4.0 R_{t n k}}\right)^{2} \\
G=2 A_{r}^{1 / 2} \cdot \frac{1-2.2 A_{r}^{1 / 2}}{1+0.2\left(H_{j e t} / D_{n}-6\right) A_{r}^{1 / 2}} \\
F_{1}=2 R e_{\text {jet }}^{1 / 2}\left(1+0.005 R e_{\text {jet }}^{0.55}\right)^{1 / 2}
\end{gathered}
$$

Where $F_{1}$ and $G$ are subfunctions composing the Nusselt number relation, $A_{r}$ is the relative area, $H_{\text {jet }}$ is the separation distance between the nozzle and impingement zone, and $r$ is the radial coordinate moving away from the stagnation point.

The ranges covered by Eqn (2-64) are as follows:

$$
\left[\begin{array}{c}
2000 \leq R e_{j e t} \leq 400,000 \\
2 \leq \frac{H_{j e t}}{D_{n}} \leq 12 \\
2.5 \leq \frac{R_{t n k}}{D_{n}} \leq 7.5 \\
\text { or } \\
0.04 \geq A_{r} \geq 0.004
\end{array}\right]
$$

\subsubsection{Submerged Liquid Jet}

[14] recommends the use of Eqn (2-70) proposed by Dominik in [25] for 1-g non-vented filling of cryogenic tankage. During the no-vent fill Eqn (2-70) models a submerged liquid jet used to mix the contents of a partially filled tank. The liquid surface impinged by the jet is assumed to deflect the flow similar to a flat plate. The Reynolds number for this case is treated with a different velocity than the average jet velocity at the nozzle exit. The jet velocity at the free surface is then calculated and used in the forming another Reynolds 
number, $R e_{x}$, in Eqn (2-68). The area of impingement, $A_{j e t}$, is used to calculate the characteristic length for use in $R e_{x}$. Modification to the average exit velocity accounts for the velocity dissipated from the jet as it moves through the liquid.

$$
\begin{gathered}
\frac{\bar{V}_{x}}{V_{\text {jet }}}=\frac{1.41 R e_{\text {jet }}^{0.135} D_{n}}{H_{j e t}} \\
R e_{x}=\frac{\rho_{j e t} V_{x} D_{j e t}}{\mu_{j e t}} \\
\overline{N u}_{j e t}=0.205 R e_{x}^{0.731} \cdot P r_{j e t}^{1 / 3}
\end{gathered}
$$

A typical CHV, beginning with a nearly evacuated tank, will not see significant liquid accumulation until the tank wall temperature approaches the saturation temperature of the liquid injections. The submerged jet model would be necessary for attempts at cooling the walls of a tank already partially full of liquid. The mixing induced by the submerged jet would elevate the heat transfer between the walls and liquid portion of the tank. Historically, a CHV of this nature has not been considered but the ability to model such a process is included in the code. 


\subsubsection{Flat Plate Natural Convection}

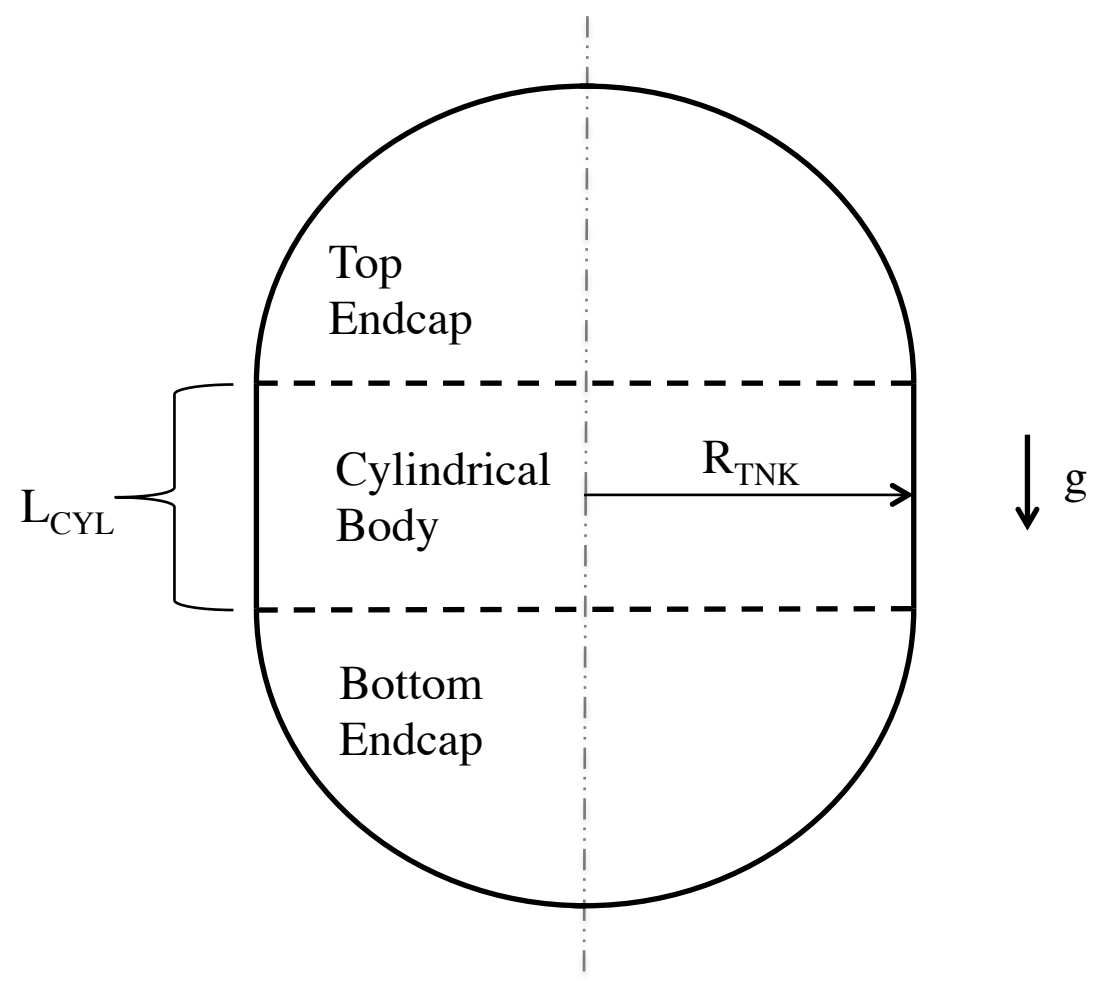

Figure 2.4: The presence of gravity differentiates the natural convection heat transfer behavior of the three sections.

Horizontal and vertical flat plate correlations compose the natural convection heat transfer relationships. Horizontal flat plates are oriented with the buoyancy force normal to the surface of heat transfer. Under 1-g conditions the position and temperature of the plate relative to the fluid influences the magnitude and behavior of the Nusselt number.

The tank geometry is approximated as three pieces as shown in Fig 2.4. Two regimes of heat transfer are modeled. First, natural convection and jet impingement are specified to occur simultaneously. Once jet impingement ceases only the natural convection modes continue to transfer heat. The top endcap, tank body, and bottom endcap are treated with separate heat transfer relations. The top endcap is assumed to behave as a horizontal flat plate cooled with fluid, via natural convection, from below. The bottom endcap is approximated as a horizontal flat plate cooled with fluid, via natural convection, from above. The 
cylindrical tank body separating the two endcaps is approximated as a vertical flat plate. The flat plate approximations preserve the area specific to each component.

The Rayleigh number and Prandtl number are required to evaluate the Nusselt number correlations. The Rayleigh number is defined as follows.

$$
\begin{gathered}
R a=\operatorname{Pr} \cdot G r \\
\operatorname{Pr}=\frac{\mu c_{p}}{k} \\
G r=\frac{g \beta\left(T_{w}-T\right) \cdot L^{3}}{(\mu / \rho)^{2}}
\end{gathered}
$$

Where $G r$ is the Grashof number, $g$ is the gravitational constant, $\beta$ is the coefficient of thermal expansivity ( $\beta=\frac{1}{T}$ for an ideal gas), and $L$ is a characteristic length scale. Fluid property values are calculated with a film temperature for the fluid and tank wall.

The characteristic length for calculation of the Rayleigh and Nusselt number for horizontally oriented plates is modified according to the recommendation of [22] and [23].

$$
L=\frac{A}{P}
$$

Where $L$ is the modified characteristic length, $A$ is the area of heat transfer, and $P$ is the perimeter of the area of heat transfer.

[24] recommends [26] and for horizontal flat plate natural convection Nusselt number correlations. A hot flat plate cooled from below is modeled by Eqn (2-75) and is valid over the range $10^{5} \leq R a \leq 10^{10}$.

$$
\overline{N u}_{t o p}=0.27 R a^{1 / 4}
$$

[24] recommends [26] and Eqn (2-76) for a hot horizontal flat plate cooled from above. 
The correlation is valid in the range $10^{7} \leq R a \leq 10^{11}$.

$$
\overline{N u}_{b o t}=0.15 R a^{1 / 3}
$$

[24] recommends [27] and Eqn (2-77) for heat transfer with a vertically oriented flat plate across the entire range of Rayleigh numbers.

$$
\overline{N u}_{c y l}=\left[0.825+\frac{0.387 R a^{1 / 6}}{\left[1+(0.492 / P r)^{9 / 16}\right]^{4 / 9}}\right]^{2}
$$

The small viscosity values of cryogenic fluids generate large Rayleigh numbers and may push the fluid state outside of the above ranges. Natural convection relations developed around cryogenics are not readily available. Therefore operating outside of the recommended range is an acceptable approximation of the natural convection heat transfer behavior of cryogenic fluids.

From the above Nusselt number correlations the heat transfer coefficients are calculated and discretized as follows.

$$
\begin{gathered}
h_{\text {jet }}=\frac{\overline{N u}_{\text {jet }} \cdot k_{\text {jet }}}{D_{\text {jet }}} \\
h_{\text {top }}=\frac{\overline{N u}_{\text {top }} \cdot k}{L_{t o p}} \\
h_{c y l}=\frac{\overline{N u}_{c y l} \cdot k}{L_{c y l}} \\
h_{b o t}=\frac{\overline{N u}_{b o t} \cdot k}{L_{b o t}}
\end{gathered}
$$




$$
\begin{aligned}
& h_{\text {jet }}^{j}=\frac{\overline{N u}_{\text {jet }}^{j} \cdot k_{s}}{D_{j e t}} \\
& h_{\text {top }}^{j}=\frac{\overline{N u}_{t o p}^{j} \cdot k^{j}}{L_{t o p}} \\
& h_{c y l}^{j}=\frac{\overline{N u}_{c y l}^{j} \cdot k^{j}}{L_{c y l}} \\
& h_{b o t}^{j}=\frac{\overline{N u}_{b o t}^{j} \cdot k^{j}}{L_{b o t}}
\end{aligned}
$$




\section{Chapter 3}

\section{Results and Discussion}

\subsection{Data Collection}

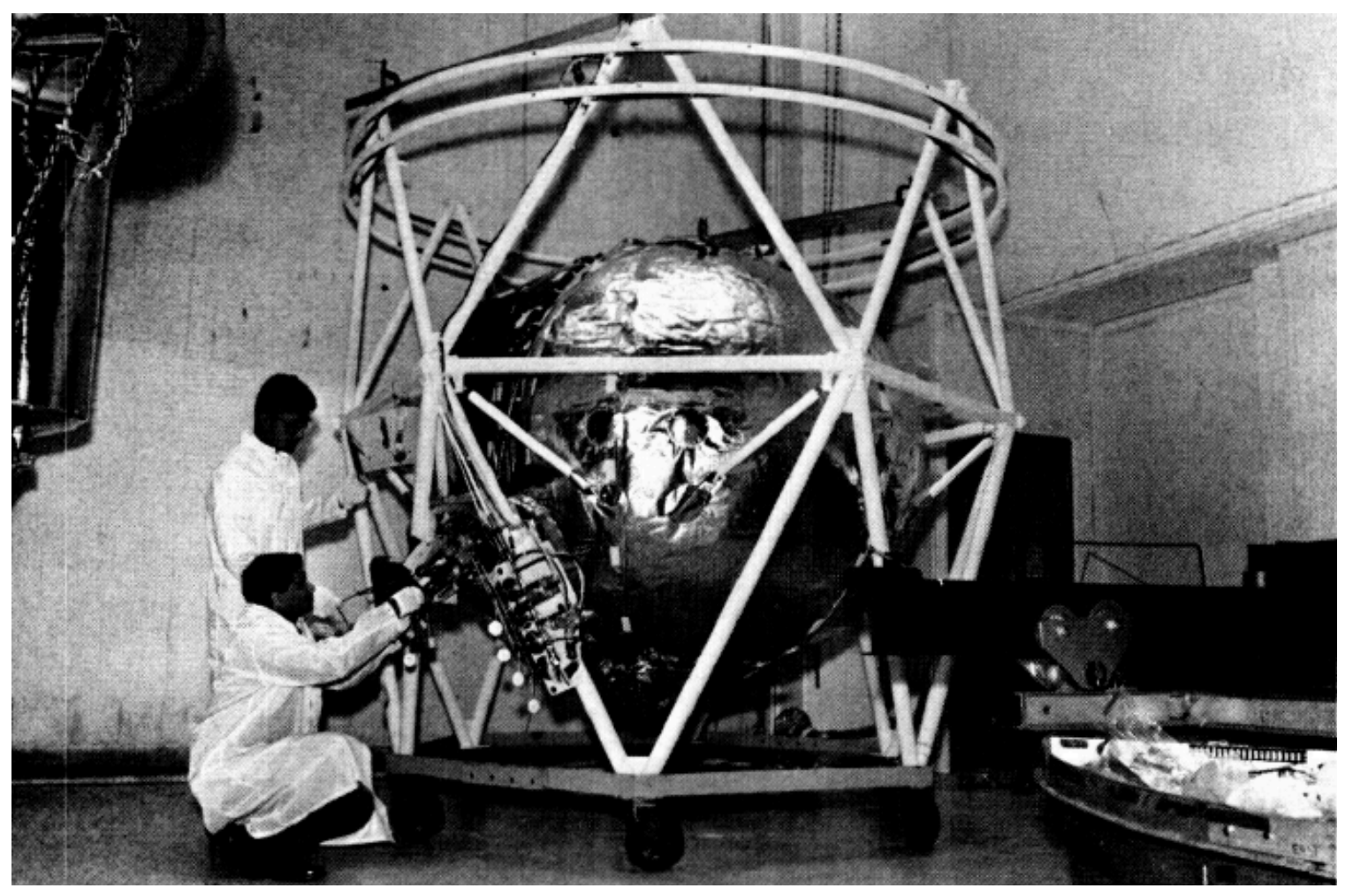

Figure 3.1: Test tank in testing structure [14]. 
Experimental data was obtained from a liquid hydrogen charge-hold-vent (CHV) performed under $1 \mathrm{~g}$ conditions in a space-like environment. Details of the experiment can be found in [8]. The test was performed at the NASA Lewis Research Center Plum Brook Station Cryogenic Propellant Tank Facility (also known as K-Site). The CHV test was run on February 15, 1991 between a series of NVF (No-Vent Fill) tests. The tank was cooled in six CHV cycles such that the intended seventh CHV cycle saw significant liquid accumulation and began the NVF phase of the test.

The tank is ellipsoidal with a $2.21 \mathrm{~m}$ major diameter and a major to minor axis ratio of 1.2. The tank endcaps are joined by a $0.04 m$ cylindrical section. The tank is made from Aluminum 2219 and has a maximum allowable working pressure (MAWP) of $344.64 \mathrm{kPa}(50 \mathrm{psia})$ and a nominal thickness of $2.21 \mathrm{~mm}$. The insulation system consists of 34 layers of multi-layer insulation (MLI) made from double aluminized mylar with silk net spacers. The tank was supported by 12 fiberglass epoxy struts as shown in Fig 3.1.

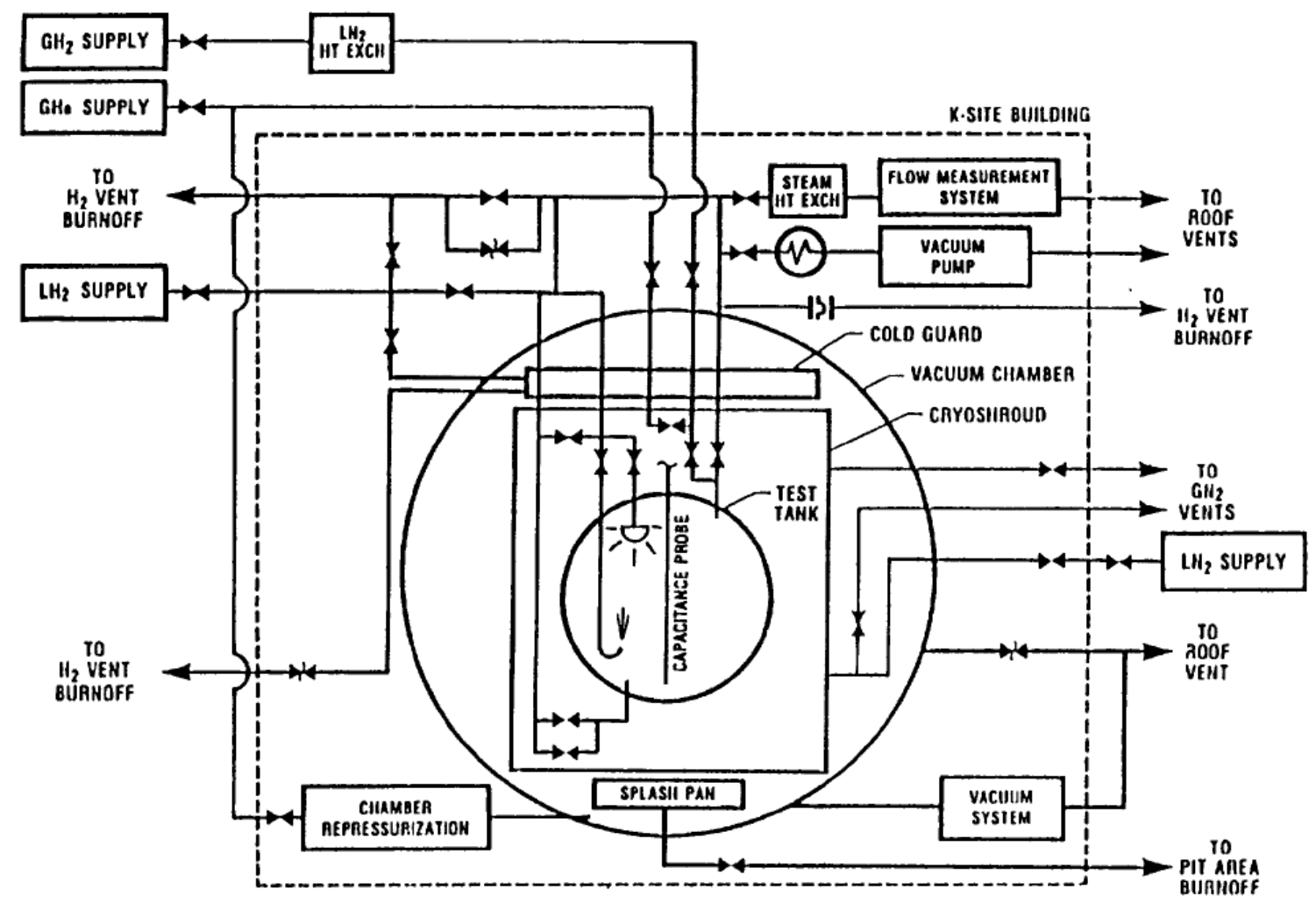

Figure 3.2: Simplified facility schematic [8]. 
The tank was fitted with two spray systems that were to be used independently during the NVF test runs. Fig 3.3 shows the shower head spray nozzle installed in the top portion of the test tank.

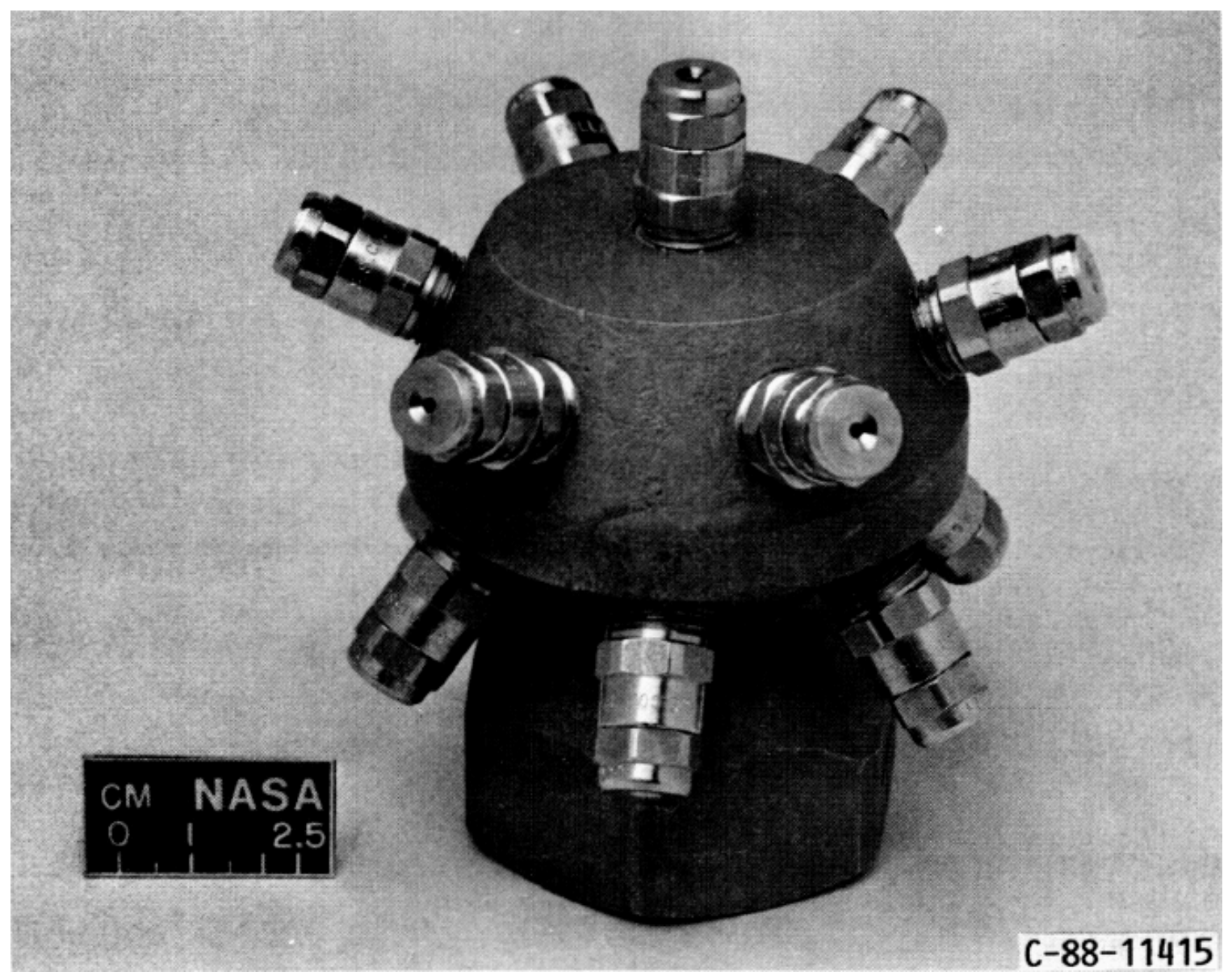

Figure 3.3: Top inlet spray nozzle. 13 full cone nozzles each with a nominal orifice diameter of $0.20828 \mathrm{~cm}$ and a water flow capacity of $0.114^{\mathrm{m}^{3}} / \mathrm{hr}$ at a $68 \mathrm{kPa}$ pressure drop. Total flow capacity is $1.48 \mathrm{~m}^{3} / \mathrm{hr}$ [14]

The top spray nozzle consisted of a shower head with 13 spray nozzles grouped together. The grouping emitted a droplet spray that was intended to promote ullage condensation during the NVF phase. Condensing the ullage vapor back into the bulk liquid allows higher fill levels to be attained. Without condensing the ullage the bulk liquid will act as a piston and compress the vapor, rapidly increasing the tank pressure and forcing a vent to occur. 
The bottom spray system is shown in Fig 3.4.

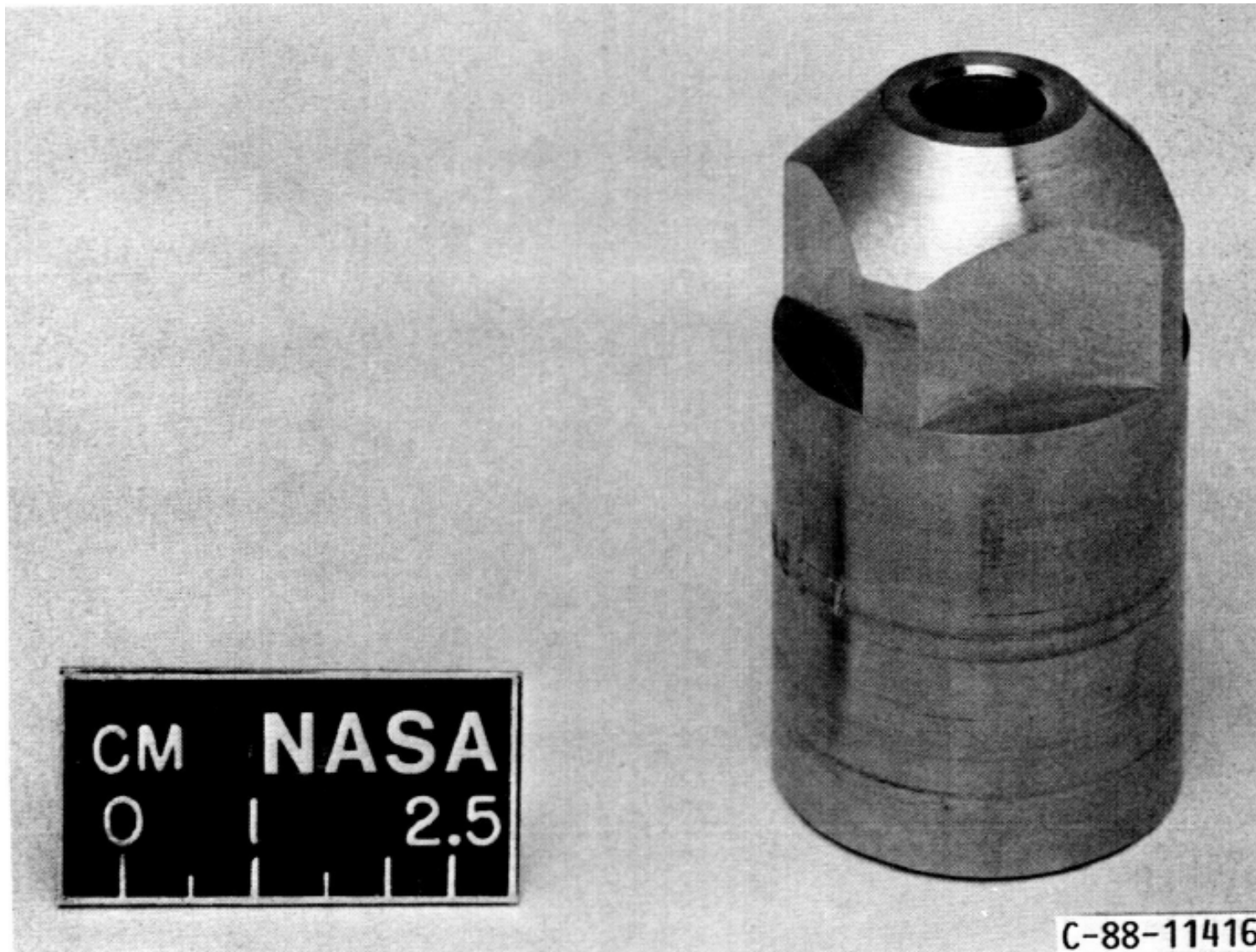

Figure 3.4: Bottom turbulent jet inlet nozzle. A single nozzle with a nominal orifice diameter of $0.9525 \mathrm{~cm}$ and a water flow capacity of $1.89^{\mathrm{m}^{3}} / \mathrm{hr}$ at a $68 \mathrm{kPa}$ pressure drop [14]

The bottom spray system consisted of a single turbulent jet. The bottom jet represented a worst case scenario for the NVF portion of the test. Its location in the tank bottom allows the nozzle to become submerged as liquid accumulates during the fill. The jet would promote ullage condensation not by spraying through the ullage vapor but by mixing the bulk liquid to prevent stratification of the its temperature distribution. The interface between the liquid and vapor will transfer mass between the two phases during the NVF which has significant impact on the tanks pressure. If the interface is left undisturbed the liquid temperatures at the liquid-vapor interface will remain high relative to the liquid temperature in lower parts of the bulk liquid. The submerged bottom jet will disturb the interface and 
encourage its temperature to drop relative to the undisturbed scenario. The temperature difference between the bulk liquid and ullage will increase and promote heat transfer between the phases which may lead to ullage condensation resulting in a tank pressure drop.

The liquid hydrogen was supplied from a $49.210 \mathrm{~m}^{3}(13,000 \mathrm{gal})$ dewar. The supply dewar was pressurized by removing a small amount of liquid hydrogen and vaporizing it. The vapor was injected back into the supply tank and the pressure increased, subcooling the bulk liquid. The thermal lag of the bulk liquid provided enough time to remove a quantity of subcooled liquid for testing. The subcooled liquid was fed through the transfer line and into the test tank. The heat leak into the transfer line was able to cause two phase flow to be present at the venturi flow meter if the liquid was not appropriately subcooled. The presence of two phase flow negatively impacted the mass flow measurements at the venturi and caused the amount of mass injected into the tank to remain unknown. Increasing the supply pressure increases the liquid subcooling and mitigates two phase flow at the venturi and tank inlet nozzles.

During the CHV experiment, both top and bottom nozzles were used to simultaneously inject mass into the tank. The test run was composed of six CHV cycles followed by a NVF (No-Vent Fill). The six chilldown cycles cooled the tank from $245 \mathrm{~K}$ to $43 \mathrm{~K}$. The NVF began on the seventh liquid injection with the wall at $45 \mathrm{~K}$ initially. Fig 3.5 details the external temperature sensors used to determine the wall temperature. 


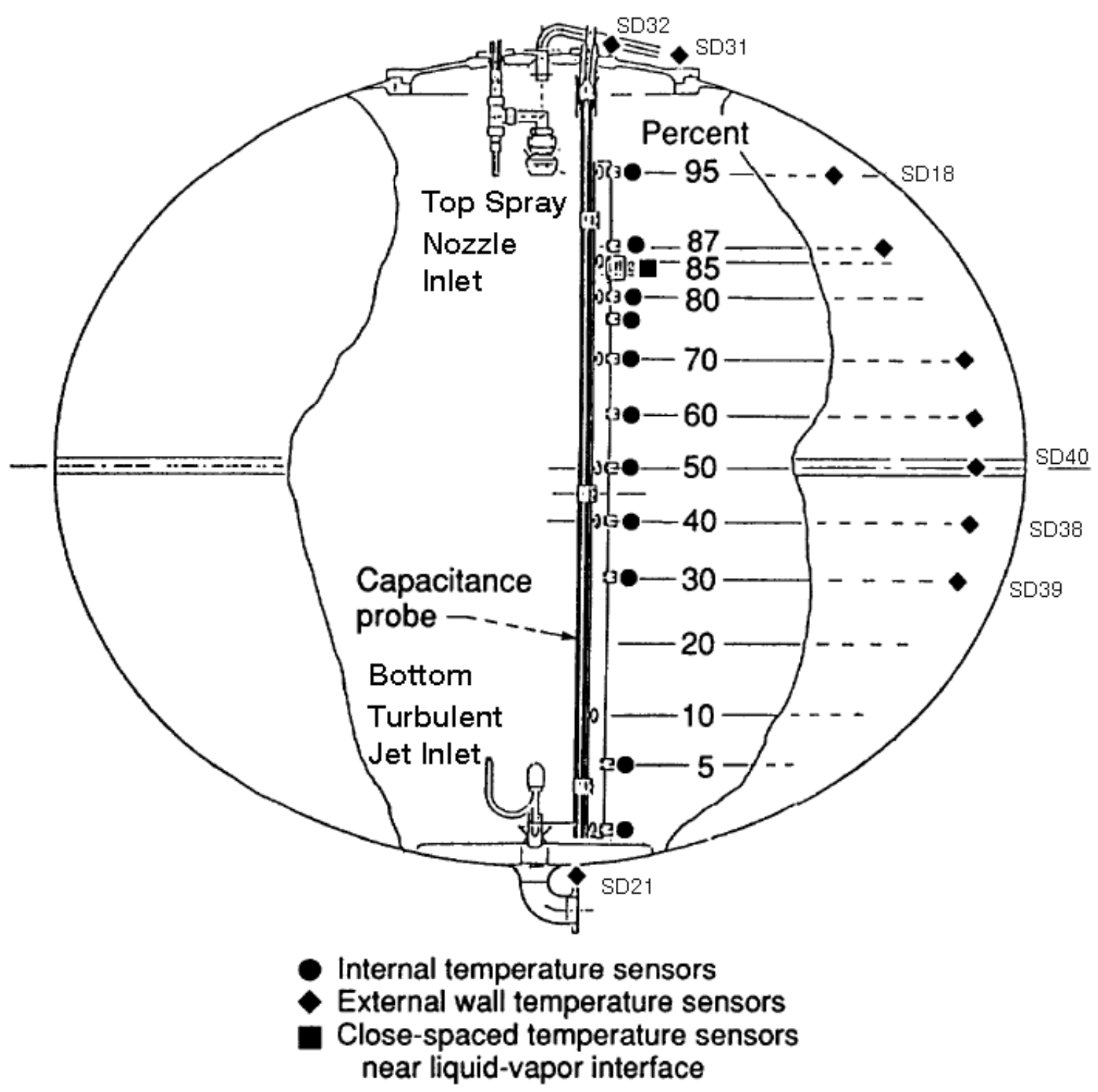

Figure 3.5: Test tank nozzle and diode arrangement [8].

A single value characterizes wall temperature as reported in [8]. This value is a weighted average of all temperature sensors including temperature measurements from the lid and bottom baffle. The bottom turbulent jet and proximity to the shower head array decreased the temperature of the top hemisphere relative to the rest of the tank wall. Fig 3.6 shows this effect during the fifth cycle. Temperature diodes SD39 and SD18 bracket the upper and lower bounds of the wall temperature, respectively. The lid remains thermally independent due to its large mass, small area, and isolation from conducting significant heat to the thin 


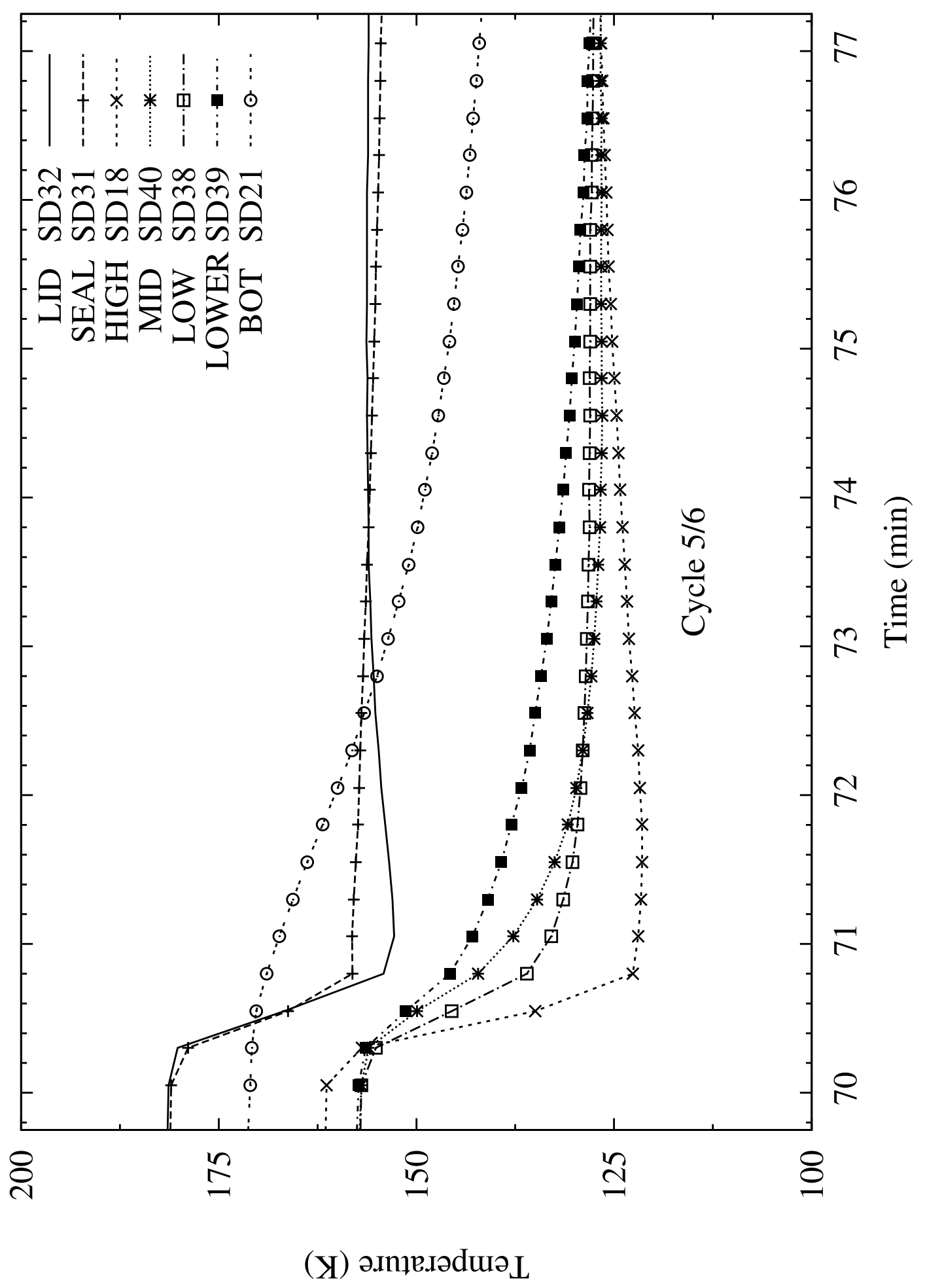

Figure 3.6: Temperature history for beginning stages of cycle 5 [8]. 
tank walls. Fig 3.7 shows the independence of the tank lid's temperature profile relative to the temperature profiles of the tank wall.

Prior to the first $\mathrm{CHV}$ cycle the tank wall, lid, seal, and baffle are in thermal equilibrium at approximately $245 K$. The lid and bottom baffle temperature profiles separated from the tank wall profiles with each successive CHV cycle. The first two cycles of the six cycle test experienced problems with two phase flow at the venturi flow meter. The supply dewar was pressurized to $137.86 \mathrm{kPa}(20 \mathrm{psi}) \mathrm{a}$ and was maintained at this pressure during the fluid transfer. The dewar pressure was increased to $172.32 \mathrm{kPa}(25 \mathrm{psi}) \mathrm{a}$ for the third CHV cycle in an attempt to subcool the supply liquid and prevent two phase flow. The increase in subcooling avoided two phase flow at the venturi but was not able to stop its occurrence at the turbine flow meters further downstream. Ultimately, the dewar pressure was increased to $206.79 k P a(30 p s i a)$ for the remainder of the test and due to a $275.71 k P a(40 p s i a)$ vent limit, could not be increased further. Two phase flow was still present after the increase to $275.71 \mathrm{kPa}(40 \mathrm{psia})$ dewar pressure but mass flow measurements were considered more reliable.

The initial injection of cold fluid drops the tank wall temperature dramatically. The drop in wall temperature is accompanied by a rise in pressure which continues until venting. Fig 3.7 and Fig 3.8 show the temperature and pressure histories for the six cycle CHV and NVF. The sixth charge induced a pressure rise that exceeded the $275.71 k P a(40 p s i a)$ limit and had to be vented sooner than the other cycles. The need to vent early was only an issue on the sixth cycle. 


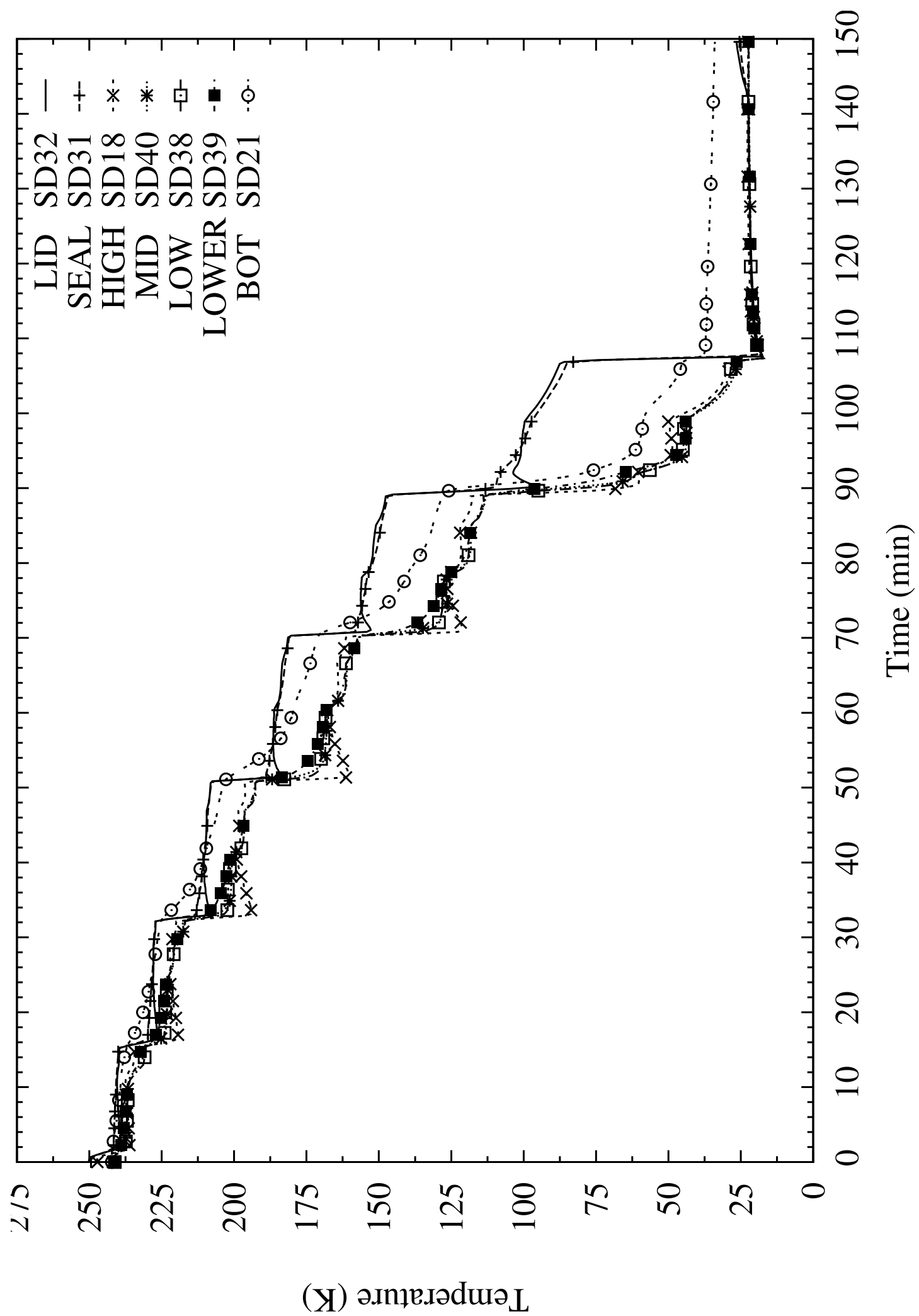

Figure 3.7: Temperature history for the 6 cycle liquid hydrogen CHV followed by a NVF [8]. 


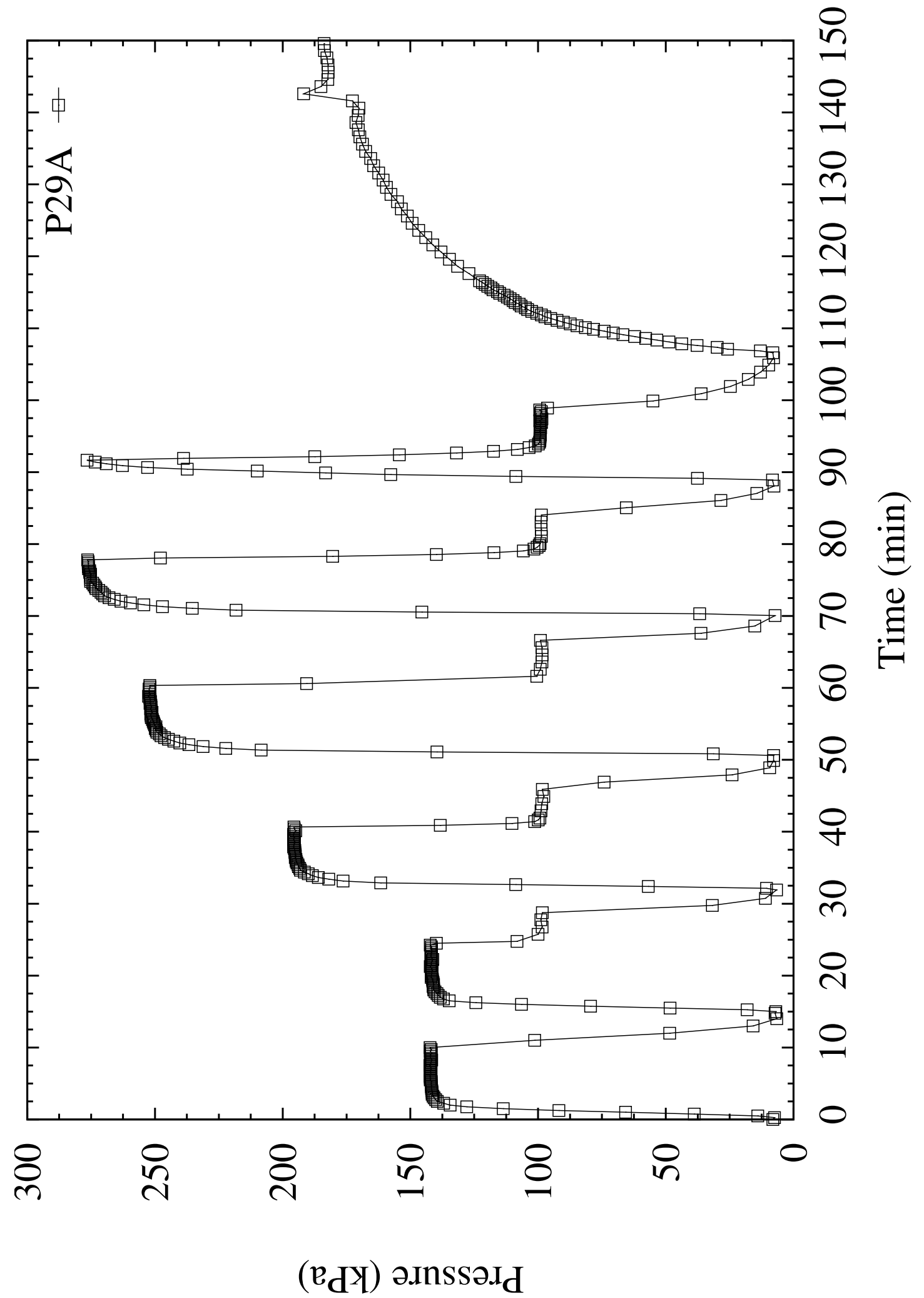

Figure 3.8: Pressure history for the 6 cycle CHV followed by a NVF [8]. Cycle 5 occurred between the 70 and 80 minute mark. 


\subsection{Model Development based on Cycle 5}

Development of the model was based on data of the fifth CHV cycle. Two phase flow was not avoided for this cycle but confidence in the mass flow measurements over the previous four charges makes cycle five more representative. The behavior of both the mass and heat transfer models are based on the pressure and temperature data from this cycle.

Temperature differences between sections of the tank are not modeled. Test data from [8] shown in Fig 3.6 supports the approximation. Temperature measurements taken from the tank body trend together. The wall, lid, and bottom baffle temperature profiles do not converge at steady state. Based on this analysis, conduction heat transfer between these parts of the tank is neglected. The heat transfer between the lid and fluid and the heat transfer between the bottom baffle and fluid are also neglected. The lid and bottom baffle are of small surface area compared to the tank wall $\left(A_{l i d} \approx A_{b a f f l e} \approx 0.4 m^{2}\right.$ compared to the wall area $A=13.86 \mathrm{~m}^{2}$ ). The bottom baffle and tank lid are neglected when determining heat transfer between the fluid and tank components.

Table 3.1 shows a summary of the cycle 5 test data. A mixture of vapor and liquid hydrogen was injected at time equals zero, the fluid was held in the tank for 7 minutes, and then a two stage venting process occurred over the last 11 minutes. The duration for fluid injection is an order of magnitude less than the duration of heat transfer (hold) and venting. The injection is approximated as instantaneous based on this analysis.

\begin{tabular}{lllll} 
Stage & $\begin{array}{l}\text { Hydrogen Mass } \\
\text { Injected }(\mathbf{k g})\end{array}$ & $\begin{array}{l}\text { Duration } \\
(\mathbf{m i n})\end{array}$ & $\begin{array}{l}\text { Final Tank } \\
\text { Pressure } \mathbf{( k P a )}\end{array}$ & $\begin{array}{l}\text { Final Wall } \\
\text { Temperature }(\mathbf{K})\end{array}$ \\
\hline Initial State & - & - & 67.55 & 165.0 \\
Charge & 2.236 & 0.750 & 217.7 & 153.5 \\
Hold & - & 7.000 & 275.4 & 134.9 \\
Vent to Atmos. & - & 6.246 & 98.29 & 127.6 \\
Vent to Vacuum & - & 4.854 & 7.996 & 123.5
\end{tabular}

Table 3.1: Cycle 5 experimental history. 


\subsubsection{Tank}

The top and bottom hemispheres are modeled as oblate spheroids with the separating section modeled as a cylinder. The surface area of the endcaps and separating section are calculated in Eqn (3-1) and Eqn (3-3).

$$
\begin{gathered}
A_{\text {top }}=A_{b o t}=\pi R_{t n k}^{2}\left[1+\frac{1-e^{2}}{e} \tanh ^{-1}(e)\right] \\
e=\sqrt{1-\frac{1}{E^{2}}} \\
A_{c y l}=2 \pi R_{t n k} L_{c y l}
\end{gathered}
$$

Where $E$ is the major to minor axis ratio of the endcaps.

The endcap areas are modeled as flat circular plates with the equivalent area of their specified actual geometry. The characteristic lengths used in Rayleigh and Nusselt number calculations are found in Eqn (3-6) and (3-7).

$$
\begin{gathered}
R_{\text {top }}=\sqrt{\frac{A_{t o p}}{\pi}} \\
R_{b o t}=\sqrt{\frac{A_{b o t}}{\pi}} \\
L_{t o p}=\frac{A_{t o p}}{P_{t o p}}=\frac{A_{t o p}}{2 \pi R_{t o p}} \\
L_{b o t}=\frac{A_{b o t}}{P_{b o t}}=\frac{A_{b o t}}{2 \pi R_{b o t}}
\end{gathered}
$$

Where $R_{t o p}$ is the radius of top endcap's flat plate representation, $R_{b o t}$ is the radius of the bottom endcap's flat plate representation, $L_{t o p}$ is the characteristic length of heat transfer for the top endcap, $L_{b o t}$ is the characteristic length of heat transfer for the bottom endcap, $P_{t o p}$ is the perimeter of the top endcap's flat plate representation, and $P_{b o t}$ is the perimeter 
of the bottom endcap's flat plate representation.

The volume of the tank was measured to be $4.955 \mathrm{~m}^{3}$ and is given in [14]. The volume may also be calculated using Eqn (3-8) and Eqn (3-9). Calculating the volume based on the given radius, major-to-minor axis ratio, and cylinder length gives a volume of $4.817 \mathrm{~m}^{3}$. The measured volume of $4.955 \mathrm{~m}^{3}$ is used in all calculations. The discrepancy in volume calculation may be attributed to measurement error and deviation of the tank endcaps away from spheroidal.

$$
\begin{gathered}
V_{t o p}=V_{b o t}=\frac{2 \pi R_{t n k}^{3}}{3 E} \\
V_{c y l}=\pi R_{t n k}^{2} L_{c y l}
\end{gathered}
$$

Table 3.2 summarizes the values used to model the tank geometry. $A_{t n k}$ was calculated

$$
\begin{array}{rl|r}
A_{t o p}=6.798 m^{2} & V_{\text {top }}=2.336 \mathrm{~m}^{3} \\
A_{\text {cyl }}=0.264 \mathrm{~m}^{2} & V_{\text {cyl }}=0.145 \mathrm{~m}^{3} \\
A_{\text {bot }}=6.798 \mathrm{~m}^{2} & V_{\text {bot }}=2.336 \mathrm{~m}^{3} \\
A_{\text {tnk }}=13.860 \mathrm{~m}^{2} & V_{\text {tnk }}=4.955 \mathrm{~m}^{3}
\end{array}
$$

Table 3.2: Tank geometry.

and $V_{t n k}$ was provided by [8]. The calculated values for $V_{t o p}, V_{c y l}$, and $V_{b o t}$ are included in the table to illustrate discrepancies between the measured value and calculated value. The values $V_{t n k}=4.955 \mathrm{~m}^{3}$ and $A_{t n k}=13.860 \mathrm{~m}^{2}$ are used in calculations when necessary.

\subsubsection{Material}

The tank is modeled as a single uniform material. The Aluminum 2219 specific heat is modeled with data tables provided by [16]. The data set is linearly interpolated to provide a continuous model of specific heat as a function of material temperature. Cycle five's material temperature range is $165 K-123.5 K$ which is in the $4 K-300 K$ range of the NIST polynomials and the $1 K-1000 K$ range of the Touloukian data tables. 


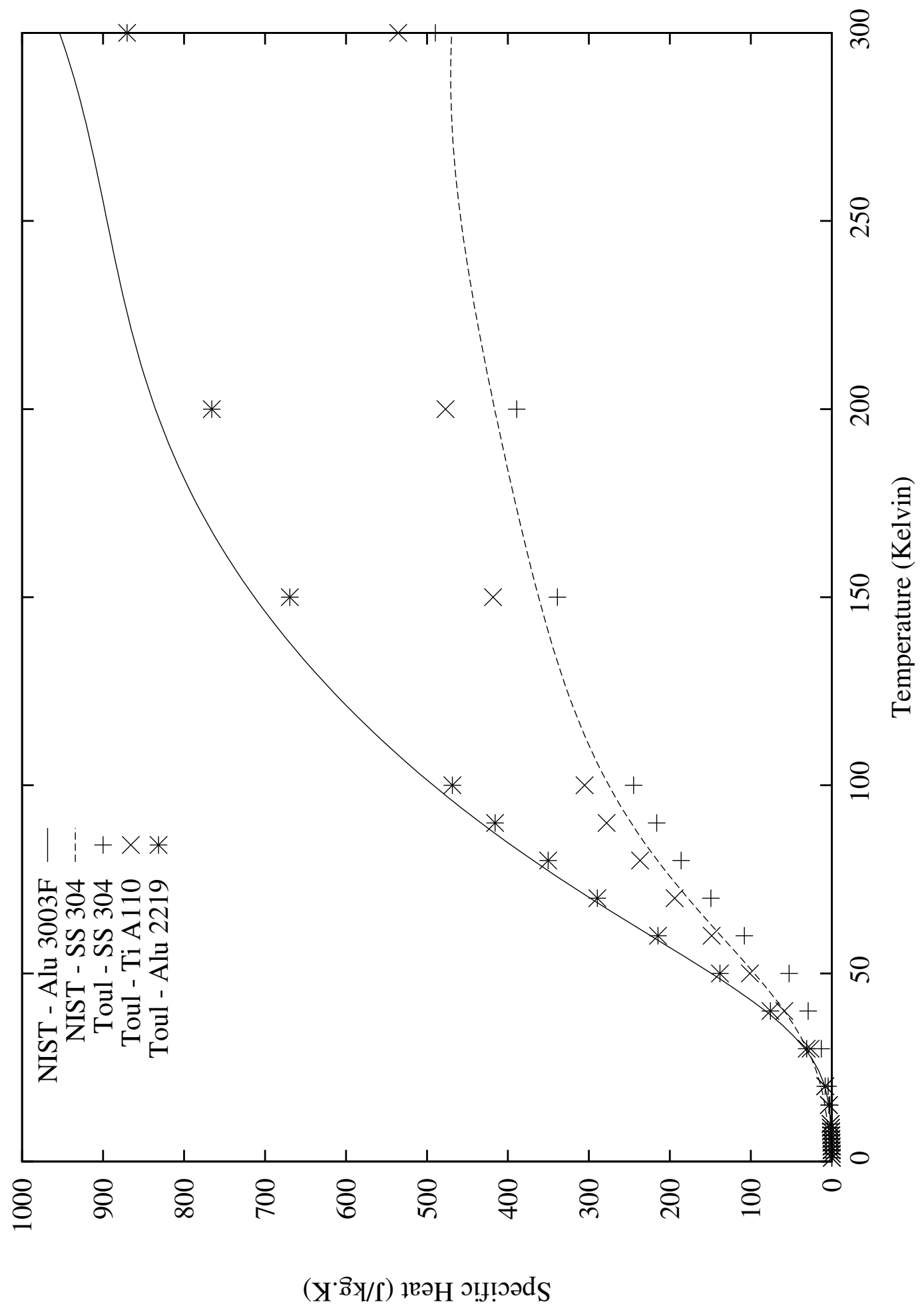

Figure 3.9: The NIST curves represent the logarithmic curve fits and the plotted points represent the data tables. The plot is limited by the range of the NIST models [16], [33], [32]. 


\subsubsection{Heat Leak and MLI Model}

[28] details the thermal performance of the $4.955 \mathrm{~m}^{3}$ tank and MLI system used during data acquisition. Two MLI blankets were used with each blanket consisting of 15 double aluminized Mylar $(0.0064 \mathrm{~mm})$ radiation shields spaced between 16 double layers of silk net spacers $\left(0.0015 \mathrm{~g} / \mathrm{cm}^{2}\right.$ per double layer) with two outer cover sheets of Schjeldahl X850 reinforced aluminized Mylar $\left(0.0078 \mathrm{~g} / \mathrm{cm}^{2}\right)$. The MLI system was approximately $23 \mathrm{~kg}\left(1.54 \mathrm{~kg} / \mathrm{m}^{2}\right)$.

Eqn (2-32) is the modified heat leak equation based on empirical data. The ambient temperature, $T_{a}$, is specified as $294.44 K$ according to the cryoshroud temperature in [8]. The insulation sheet emissivity is specified as the emissivity at $294 K$ and is constant for all heat leak calculations. [28] recommends the following values for the remaining parameters in Eqn (2-32): Heat leak is calculated as $12.1 W$ at its maximum.

$$
\begin{array}{llll}
C_{s}= & 8.95 E^{-8} & \epsilon= & 0.053 \\
C_{R}= & 5.39 E^{-10} & \bar{N}= & 17.7 \text { layer } / \mathrm{cm} \\
C_{p}= & 3.67 E^{2} & N_{S}= & 30 \text { layers }
\end{array}
$$

Table 3.3: Empirical heat leak equation parameters. 


\subsubsection{Charge}

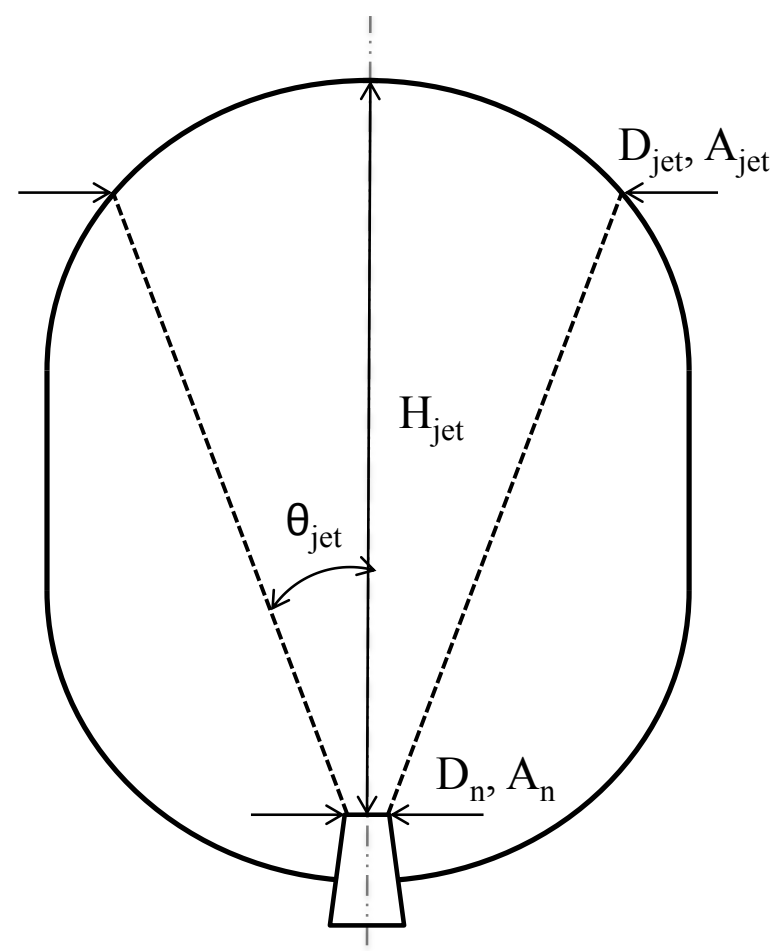

Figure 3.10: Simulation jet configuration.

The state of the tank before mass injection is specified by $P^{0}$ and $T_{w}^{0}$. Care must be taken to specify the initial tank state above the triple point of the transferring fluid. Temperatures and pressures near the triple point would cause the fluid to freeze onto valves and other tank hardware which may cause complications during the chilldown and fill. The initial state of the tank for cycle 5 is specified as $\left(T_{w}=170 K, P^{0}=7 k P a\right) .(T=13.84 K, P=$ $7.04 k P a$ ) is the triple point for hydrogen.

The enthalpy of the fluid inside the tank before mass injection, $h^{0}$, and the initial density of the tank contents, $\rho^{0}$, are calculated at $\left(T_{w}, P^{0}\right)$ and are accounted for during injection.

$$
\begin{gathered}
h=\frac{h^{0} \cdot \rho^{0} \cdot V_{t n k}+m_{i} \cdot h_{s}}{\rho^{0} \cdot V_{t n k}+m_{i}} \\
m=m_{i}+\rho^{0} \cdot V_{t n k}
\end{gathered}
$$


Liquid accumulation may occur following injection and before heat transfer. Provided the state of fluid falls within the vapor dome a two phase mixture will exist.

The nozzle arrangement is simplified by approximating the total number of nozzles as a single nozzle impinging a single area from a single location in the tank. $A_{j e t}$ is specified but a range of jet half angles $\left(7.5^{\circ}\right.$ to $\left.20^{\circ}\right)$ is recommended for free turbulent jets by [29]. The area impinged by the jet is approximated as circular and flat. The jet half angle, $\theta_{j e t}$, is chosen as $16.75^{\circ}$ and the jet impingement area and diameter are calculated. $R_{j e t}$ and $A_{j e t}$ are found to be $0.564 \mathrm{~m}$ and $1.0 \mathrm{~m}^{2}$ at a separation distance of $H_{\text {jet }}=1.875 \mathrm{~m}$. The jet is simulated as positioned at the bottom of the tank and the addition of the minor radii of the oblate spheroidal endcaps and the height of the cylindrical separating section gives the separation distance, $H_{j e t}$.

$$
\begin{gathered}
A_{j e t}=\pi R_{j e t}^{2} \\
R_{j e t}=H_{j e t} \tan \left(\theta_{j e t}\right) \\
\theta_{j e t}=\tan ^{-1}\left(\frac{R_{j e t}}{H_{j e t}}\right)
\end{gathered}
$$

Mass injection is assumed to occur instantaneously and at a constant mass flow rate across a time step. A time scale for liquid impingement is determined in Eqn (2-6) by the specified average mass flow rate and the specified injected mass. The tank pressure is compared to the supply pressure at the end of each time step. Once the supply pressure is exceeded by the tank pressure, the jet is turned off. $t_{j e t}$ is the duration the jet is turned on and is determined by comparing the supply and receiver tank pressures at the end of each timestep. Once the receiver tank pressure exceeds the supply pressure, the jet is turned off and the final calculated jet velocity is stored in $V_{j e t, c} . V_{j e t, c}$ estimates the level of fluid motion and, therefore, convective heat transfer that persists in the tank after the jet is turned 
off. $V_{j e t, c}$ is then driven to zero by the patching function shown in Eqn (3-15).

$$
\begin{gathered}
V_{j e t}=V_{j e t, c}\left[0.00001+0.501\left(1-\operatorname{erf}\left(4 \tau_{t}-1.8\right)\right)\right], P_{s}<P \\
\tau_{t}=\frac{N_{\Delta t}-N_{\Delta t_{j e t}}}{N_{\Delta t_{i}}-N_{\Delta t_{j e t}}} \\
N_{\Delta t_{i}}=\frac{t_{i}}{\Delta t} \\
N_{\Delta t_{j e t}}=\frac{t_{j e t}}{\Delta t}
\end{gathered}
$$

Where $V_{j e t, c}$ is the jet velocity calculated when $N_{\Delta t}=N_{\Delta t_{j e t}}, N_{\Delta t_{j e t}}$ is the number of time steps the jet is turned on, $t_{j e t}$ is the amount of time the jet is turned on, $N_{\Delta t_{i}}$ is the number of time steps in the injection phase. $N_{\Delta t_{j e t}}$ is calculated once $P_{s}<P$. Eqn (3-15) is plotted in Fig 3.11 . 


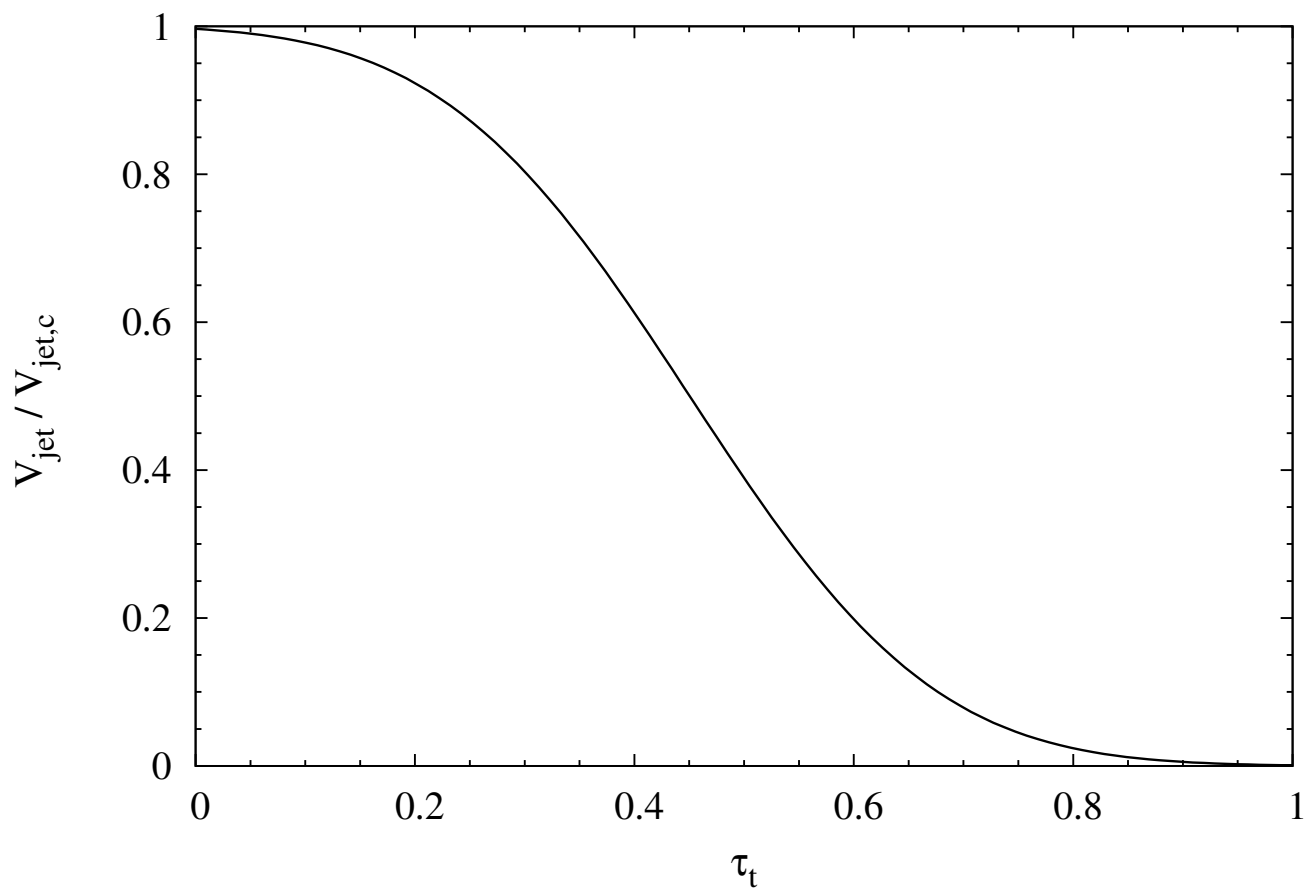

Figure 3.11: Decaying behavior imposed on $V_{\text {jet }}$ following equilibration of receiver tank pressure and supply tank pressure $\left(P>P_{s}\right) . V_{\text {jet }, c}$ is the calculated nozzle velocity from the timestep before pressure equilibration.

The injection time, $t_{i}$, is an estimate of the duration of jet impingement. To account for transitioning heat transfer that persists past $t_{i}$ an additional time scale is used to extend $t_{i}$. $t_{t}$ is estimated by a dimensional analysis shown in Eqn (3-19).

$$
t_{t}=\frac{m_{i}}{\dot{m}_{i}} \frac{D_{\text {jet }}}{H_{\text {jet }}}=t_{i} \frac{D_{\text {jet }}}{H_{\text {jet }}}
$$

Transition from jet impingement begins as soon as the receiver tank pressure and the supply tank pressures equilibrate.

The jet only transfers heat with the tank wall. No heat transfer occurs between the jet and the tank contents it passes through before it contacts the tank wall. The jet impinges a specified area of the tank, $A_{j e t}$, at a specified separation distance, $H_{j e t} . A_{j e t}$, and $H_{j e t}$ are constant during injection. $H_{\text {jet }}$ is a factor when calculating the gas jet Nusselt number 
correlation from Eqn (2-64) and when calculating $\theta_{j e t}$ from Eqn (3-14). After investigation of the three heat transfer models available in the code it was found that Eqn (2-63) best captures the transient behavior of jet impingement for the 5th CHV cycle. The Nusselt number correlation developed by Jiji in [20] and shown in Eqn (2-63) models the jet heat transfer behavior during the fifth $\mathrm{CHV}$ cycle.

It is recommended in [20] that jet properties are evaluated at a film temperature. The low pressure environment of the receiver tank causes the state of the jet near the area of impingement heat transfer to be uncertain. The jet properties are found at the nozzle inlet where its state is specified as the supply tank state. Fluid properties for the Nusselt number correlations are evaluated at supply conditions and remain constant. Heat transfer with the jet is calculated with the jet film temperature and wall temperature. The jet Reynolds number decays with time according to the jet velocity described in Eqn (3-15) while the jet Prandtl number remains constant.

$$
\begin{gathered}
R e_{j e t}=\frac{\rho_{j e t} V_{j e t} D_{h}}{\mu_{j e t}}=\frac{\rho_{s} V_{j e t}^{j} D_{n}}{\mu_{s}} \\
P r_{j e t}=\frac{\mu_{j e t} c_{p, j e t}}{k_{j e t}}=\frac{\mu_{s} c_{p, s}}{k_{s}}
\end{gathered}
$$

The wall is modeled as a single temperature node devoid of temperature gradients. The tank wall cools uniformly despite the local nature of jet impingement heat transfer. The area impinged by the jet does not differ in temperature from the rest of the tank. During jet heat transfer the rates of heat transfer experienced by the entire tank wall control volume are elevated over the heat transfer rates of natural convection. Varying the area impinged by the jet will change the rate of heat transfer between the wall and fluid but will not affect a temperature gradient in the tank wall.

If the elapsed time of the current chilldown cycle is less than $t_{i}$ impingement heat transfer is present in addition to natural convection. After the elapsed time exceeds $t_{i}$ only natural convection remains in effect. Transitioning from impingement and natural 
convection heat transfer to solely natural convection heat transfer must be smooth. A sharp discontinuity will exist in the model's output if numerical treatments for smoothing the transition are not implemented. This discontinuous behavior is not physical and is a result of assuming the impingement heat transfer ceases instantaneously. Velocity decay and persisting motion induced by the fluid injection are not modeled but their effects on heat transfer behavior are present in the experimental data. A curve fit between the two phases of heat transfer smooths the behavior across a provided timescale, $t_{t}$. Fig 3.13 shows output of the code without the use of a patching function.
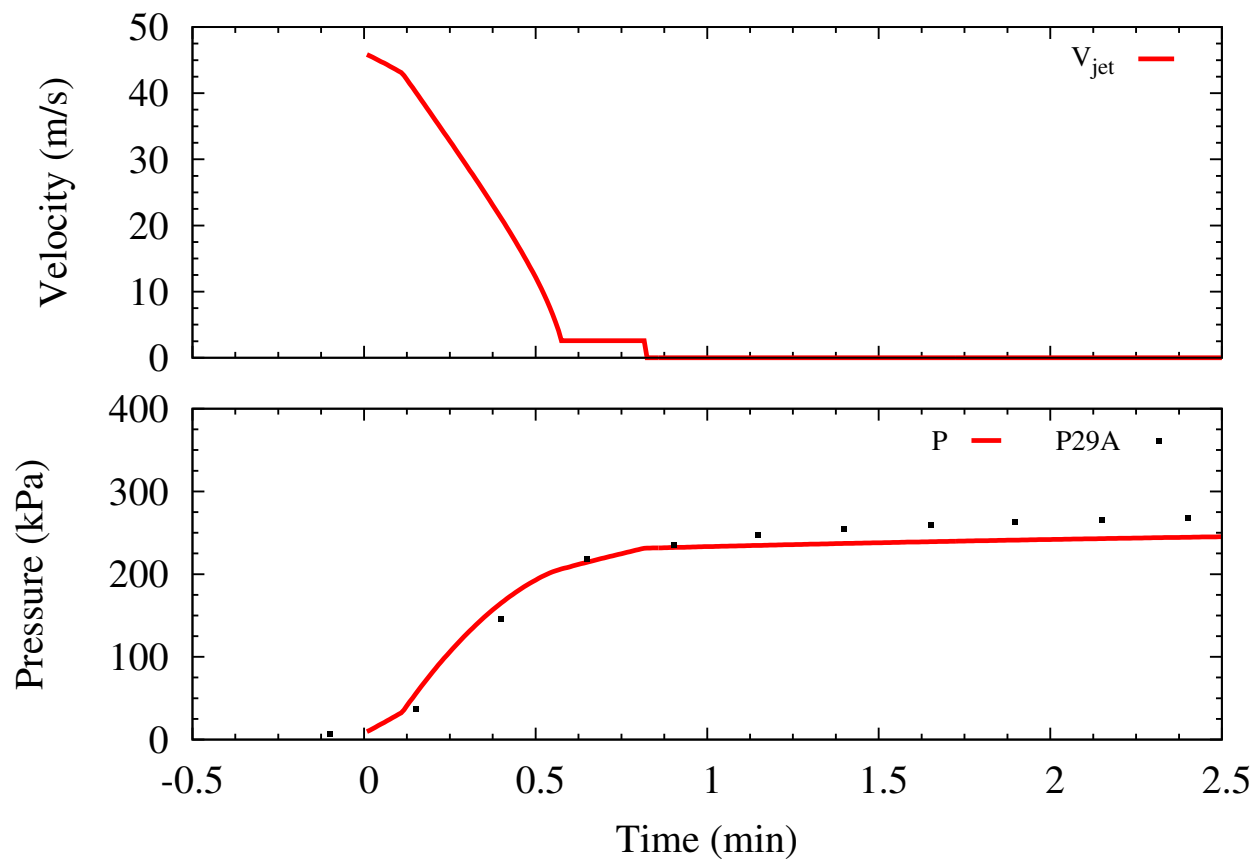

Figure 3.12: No imposed patching function and transition time.The velocity behavior once the jet is turned off instantaneously drops to zero once the injection time is exceeded.

The patching function accounts for the real transient behavior of using flow control hardware. Valves cannot be instantaneously turned on or off. Fluid remaining in the lines between the tank and valve 2501 will continue to flow into the tank at a reduced rate, eventually decaying to zero. The patching function imposes a decaying behavior on the jet 


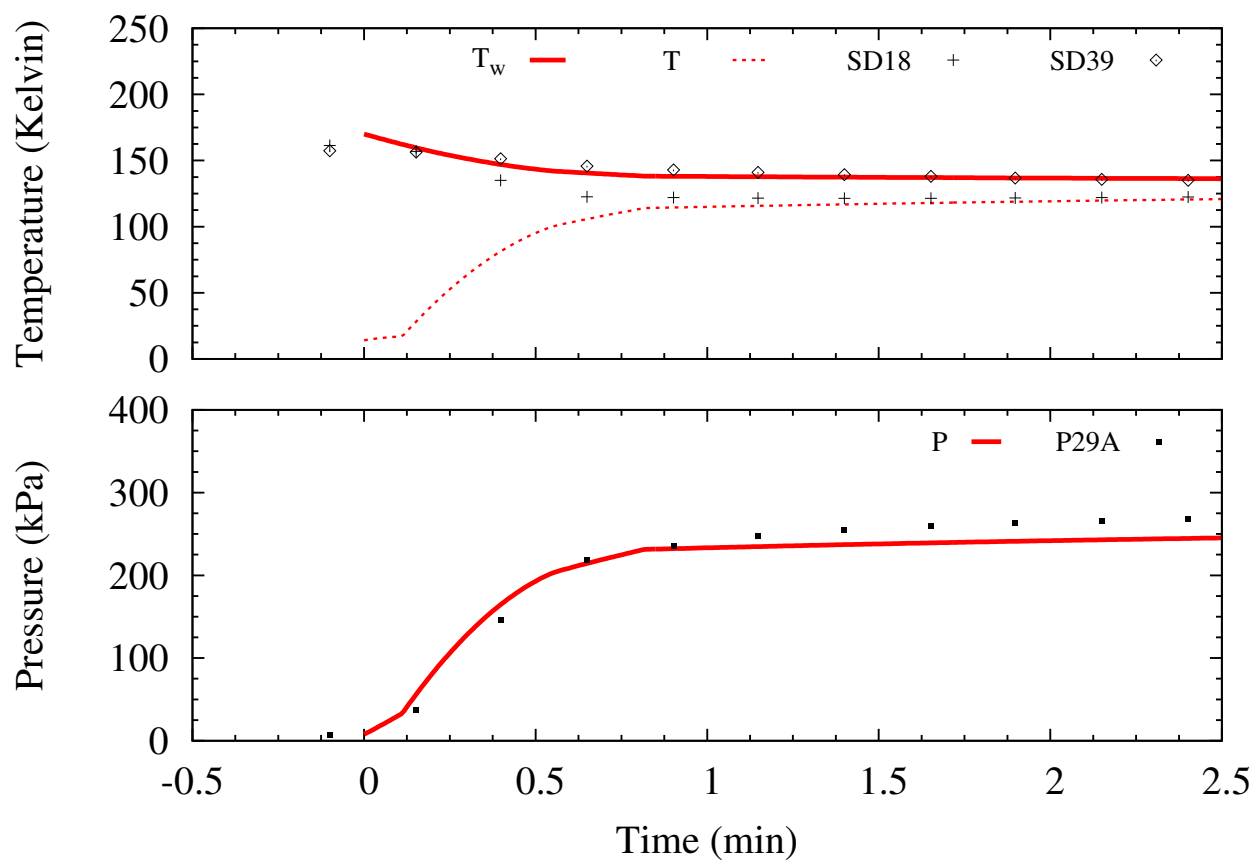

Figure 3.13: No transition from jet.

heat transfer mode to account for these effects. Flow control hardware details are shown in Fig 3.14.

The decaying behavior of heat transfer in low gravity conditions is attributed to the physics of persisting fluid motion initially induced by the inertia of the injected fluid. The forced convection heat transfer of cryogenic fluid injected into a tank in a low gravity environment is analyzed in [34]. The patching function captures the general behavior of the CFD analysis while remaining computationally inexpensive, simple to implement, and easy to understand and modify.

Honkonen [12], [34] studies the effects of fluid persistence in low gravity CHV chilldowns. Honkonen states the heat transfer behavior of fluid in micro gravity heavily dependent on nozzle orientation with in the tank. Radial injection induces chaotic motion within the tank and the Nusselt number decays around 10s. Tangential injection induces an 


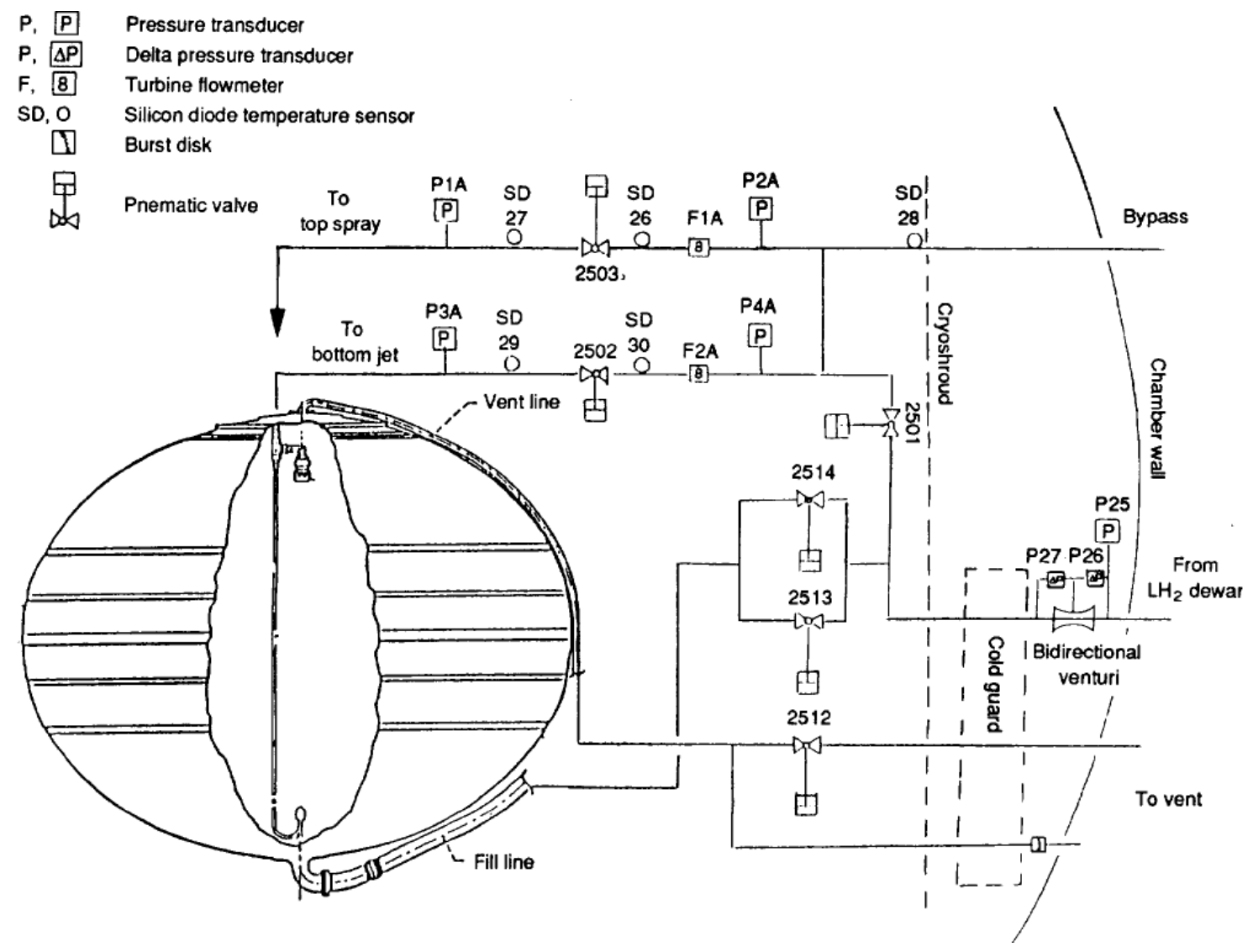

Figure 3.14: Details of hydrogen lines and instrumentation inside test chamber [8].

organized swirling flow pattern inside the tank. Fluid motion associated with this injection configuration persists longer relative to radial injection. The General Dynamics persistence model predicts the tangential injection to persist for four times longer than the radial injection. The injection method for the K-Site data cannot be characterized as radial or tangential. The use of both a turbulent jet inlet nozzle and a spray inlet nozzle would place the expected Nusselt number decay rate between that of the tangential and radial injection schemes for a low gravity simulation.

Honkonen summarizes that the greatest difference between radial and tangential injection occurs in low gravity environments. The effects of natural convection under normal gravity conditions make the system less sensitive to nozzle configuration. GDNVF uses finite difference techniques and the Crank-Nicholson method to formulate the governing 
equations into an implicit set of difference equations. A mesh with variable spacing in the radial direction. Time steps were calculated by conserving angular momentum at each mesh point. The results of this code were used in the development of the Cryogenic OnOrbit Liquid Depot Storage, Acquisition, and Transfer Satellite (COLD-SAT) flight hardware. The COLD-SAT tank was $0.56 \mathrm{~m}^{3}$ according to [35].

The transition time, $t_{t}$, is determined from two separate analysis, the injection time for cycle 5, Eqn (3-19), and the analysis of low gravity cryogenic fluid persistence provided by Honkonen. The injection time for cycle 5 was calculated as 49 s from the approximate nozzle. Based on Honkonen's CFD analysis of two nozzle geometries for a liquid hydrogen tank chilldown in low-gravity $t_{t}$ is to be on the order of 10s. The top and bottom spray systems of [8] do not induce an organized flow upon their cessation. Therefore the time for fluid persistence and velocity decay with in the $4.955 \mathrm{~m}^{3}$ tank will be less than the velocity decay in the $0.56 \mathrm{~m}^{3}$ COLD-SAT tank. A value of $30 \mathrm{~s}$ was chosen as the transition time for CHV cycle 5. 


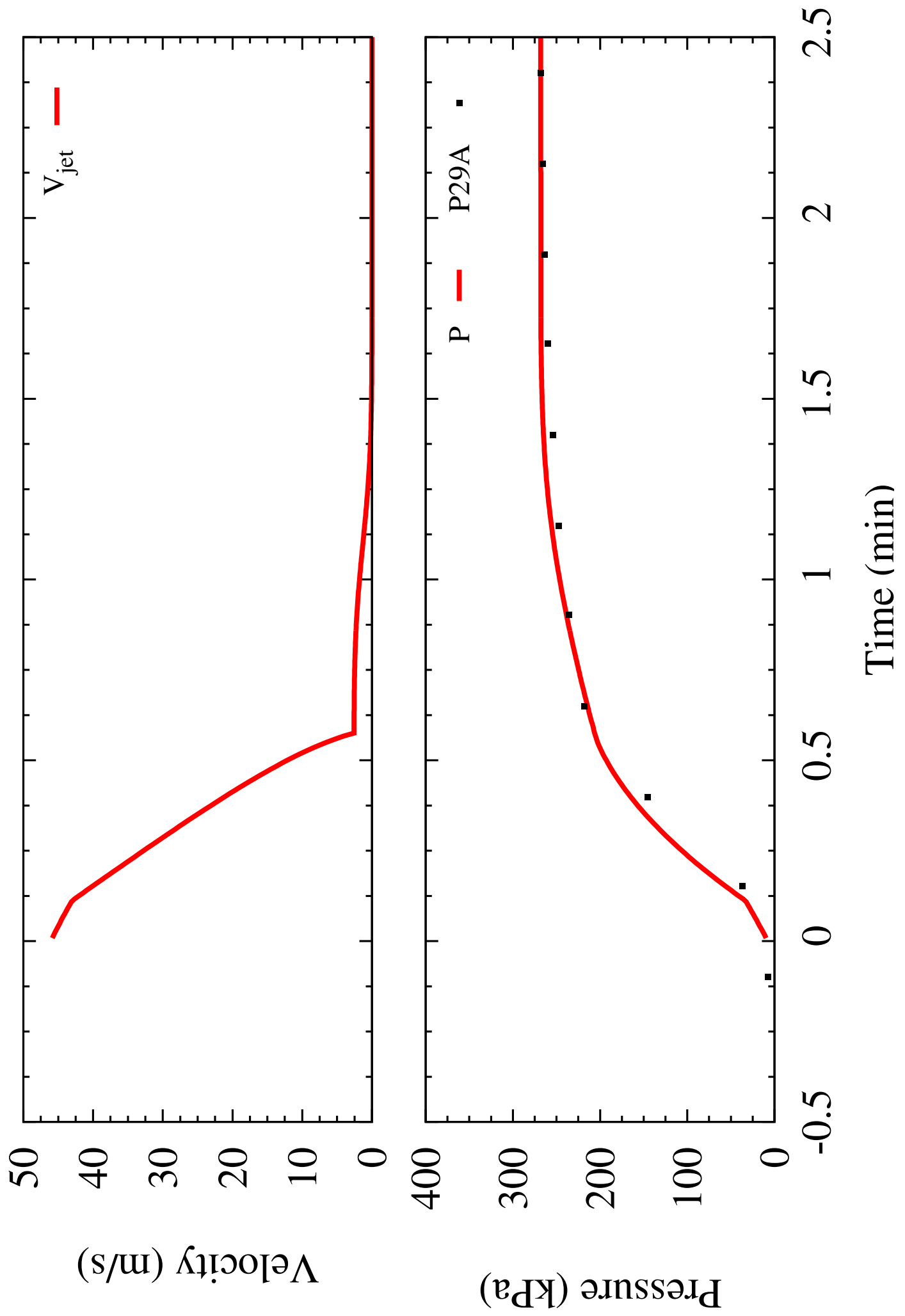

Figure 3.15: The velocity decays to zero once the jet is turned off. Additional time, estimated by Eqn (3-19), is added to the injection time to smooth the decay. 


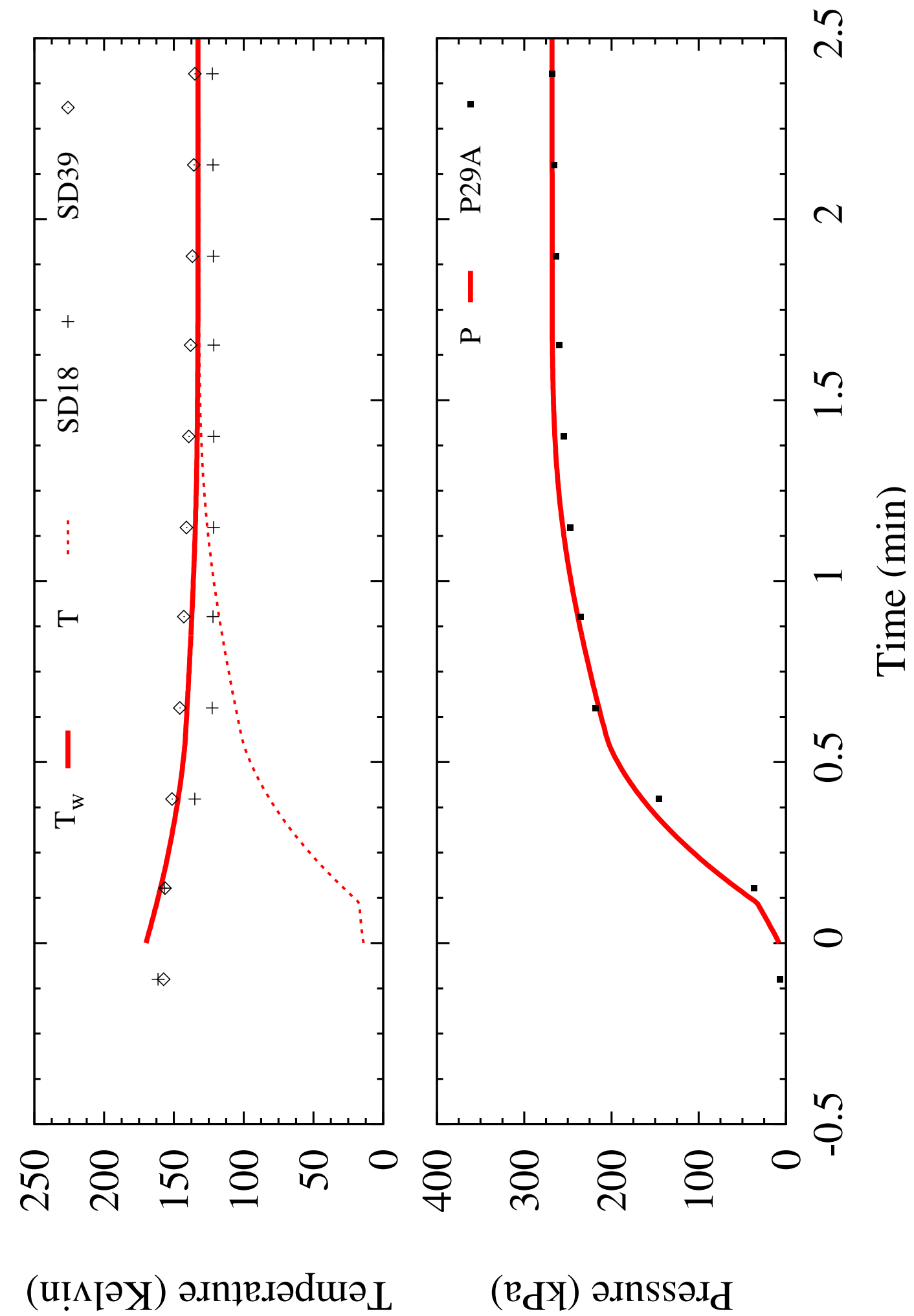

Figure 3.16: Pressure history compared against tank wall and fluid temperature histories influenced by the functional behavior imposed on jet velocity. 


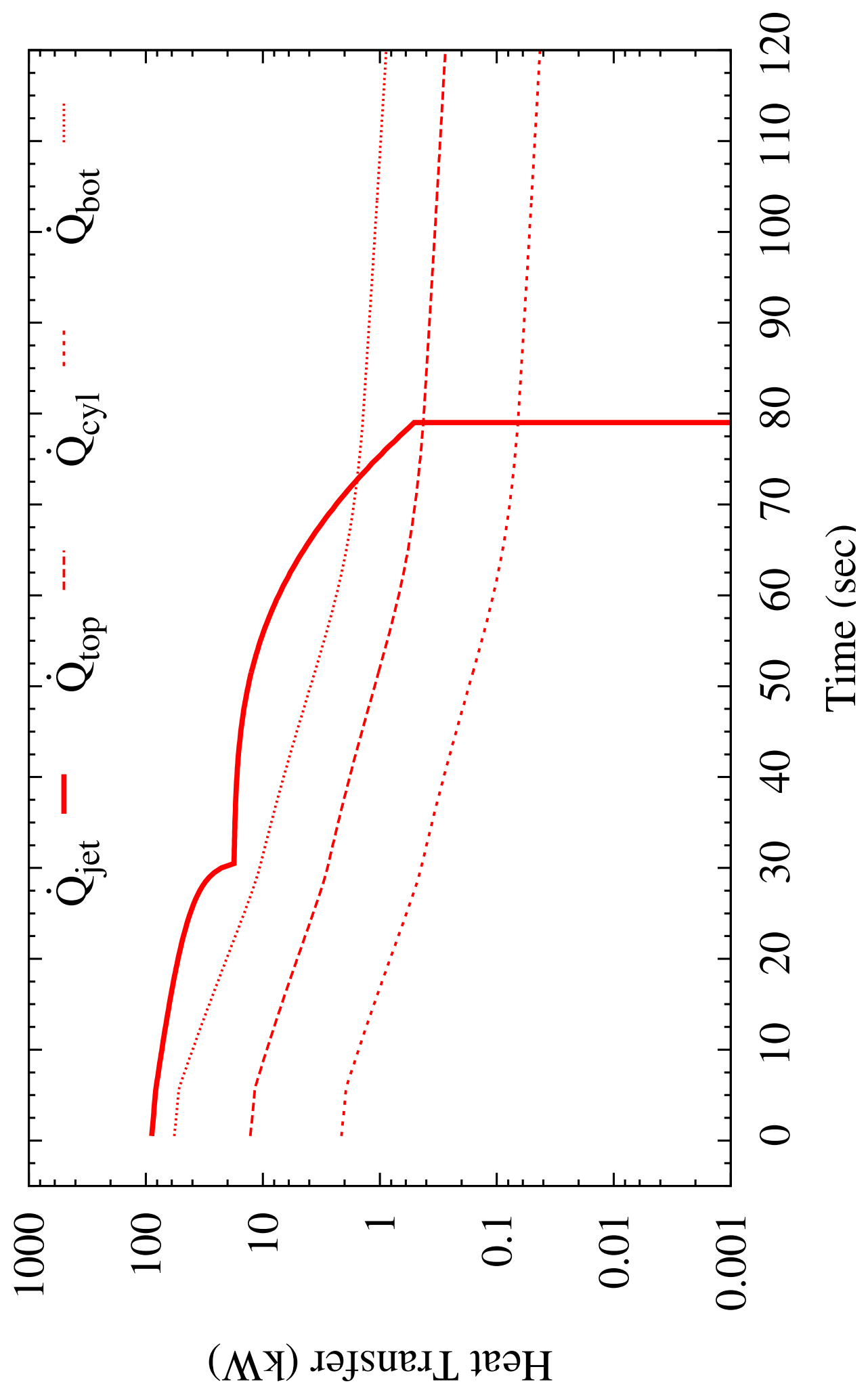

Figure 3.17: Heat transfer behavior due to natural convection and jet impingement during the charge phase. Patching function is imposed on the nozzle velocity. 


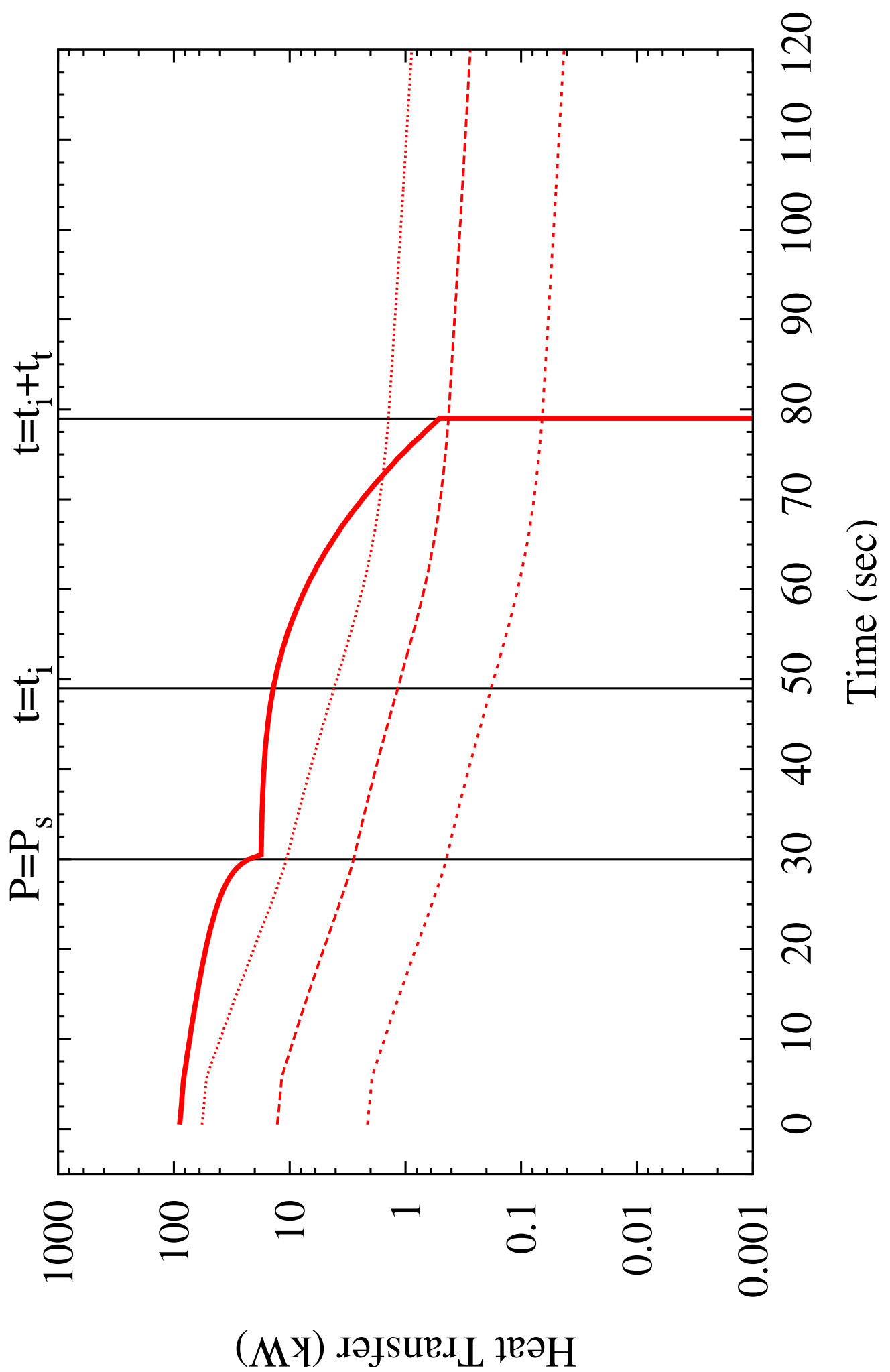

Figure 3.18: $P=P_{s}$ the jet is turned off and $V_{j e t, c}$ is calculated. $V_{j e t, c}$ decays according to Eqn (3-15) until $t=t_{i}+t_{t}$. If $t_{t}=0.0$ then $V_{\text {jet }, c}$ decays until $t=t_{i}$ 


\subsubsection{Hold}

The hold phase is defined as elapsed time greater than the sum of injection time and transition time $\left(t_{e}>t_{i}+t_{t}\right)$. During this phase only natural convection heat transfer is present. The top and bottom ellipsoidal endcaps of the $4.955 \mathrm{~m}^{3}$ tank are modeled as flat plates of the same material and area, $A_{t o p}=A_{b o t}=7.31 \mathrm{~m}^{2}$.

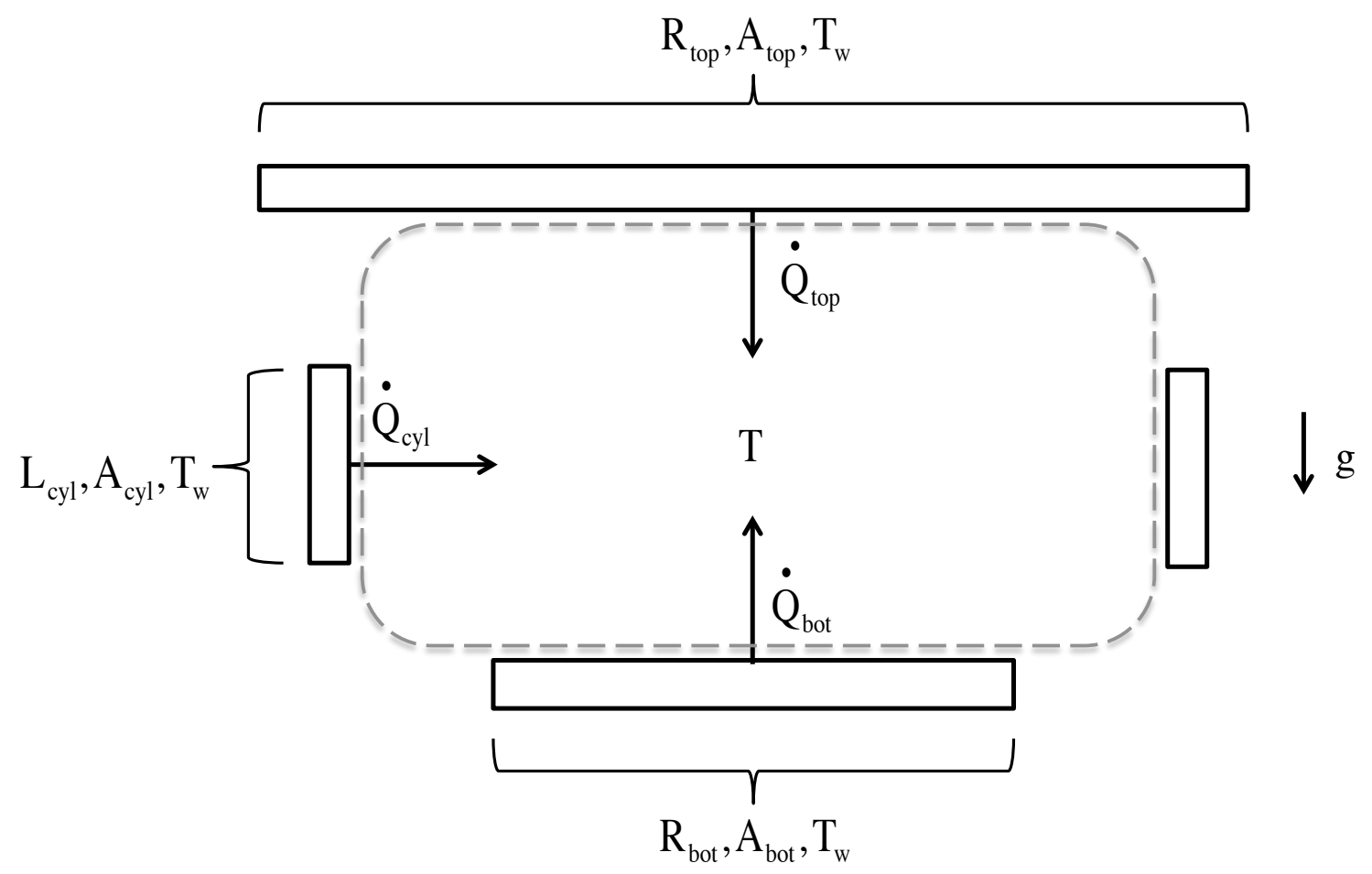

Figure 3.19: The top and bottom endcaps do not have to be of the same geometry. The differences in area are accounted for by varying the flat plate representations of each endcap.

\subsubsection{Vent}

The vent phase begins once the temperature difference between the vapor and wall is reached or once the maximum number of time steps allotted for this phase, $N_{\Delta t, \text { max }}$, is exceeded. The number of parent vent cycles is specified along with the desired amount of mass to vent during each parent vent cycle. The vent mass scaling constant, $m_{o}^{1}$, is solved for using a one dimensional Newton Raphson method and adapts each parent vent process 
to provide the specified total vent mass.

The parent vent cycles are next divided into several smaller subvent cycles. The subvent cycles contain an instantaneous mass removal followed by a hold phase which allows heat to transfer to the isentropically cooled fluid. The duration of each sub-vent phase is constant across the parent vent and determines the duration of the parent vent. The duration of each sub-vent is determined by the number of time steps per sub-vent $N_{\Delta t / s u b-v e n t}$. The duration of the parent vent is determined by the time steps per sub-vent multiplied by the number of sub-vents per parent vent, $N_{\text {sub-vent, } \max }$.

$$
\begin{gathered}
m^{j+1}=m^{j}-m_{o}\left(\tau_{o}\right) \\
m_{o}\left(\tau_{o}\right)=m_{o}^{1}\left[0.05+0.51 \cdot\left(1-\operatorname{erf}\left(3 \tau_{o}-1\right)\right)\right] \\
\tau_{o}=\frac{N_{\text {sub-vent }}}{N_{\text {sub-vent }, \text { max }}}, 0<\tau_{o} \leq 1
\end{gathered}
$$

Where $m^{j}$ is the mass in the tank before a sub-vent occurs, $m_{o}\left(\tau_{o}\right)$ is the amount of mass vented for each subvent and changes with each passing subvent, $m_{o}^{1}$ is a scaling constant for the vent process, $N_{\text {sub-vent }}$ is the number of the current subvent, $N_{\text {sub-vent,max }}$ is the total number of sub-vents composing the parent vent, and $\tau_{o}$ is dimensionless time for the current parent vent. For a fixed $N_{d t / s u b-v e n t}$ and $\left.m\right) o^{1}$, increasing $N_{\text {sub-vent,max }}$ will increase the number of sub-vents which increases both the duration of the parent vent and the amount of mass vented.

Fig 3.20 illustrates the models behavior when mass is permitted to vent instantaneously. 


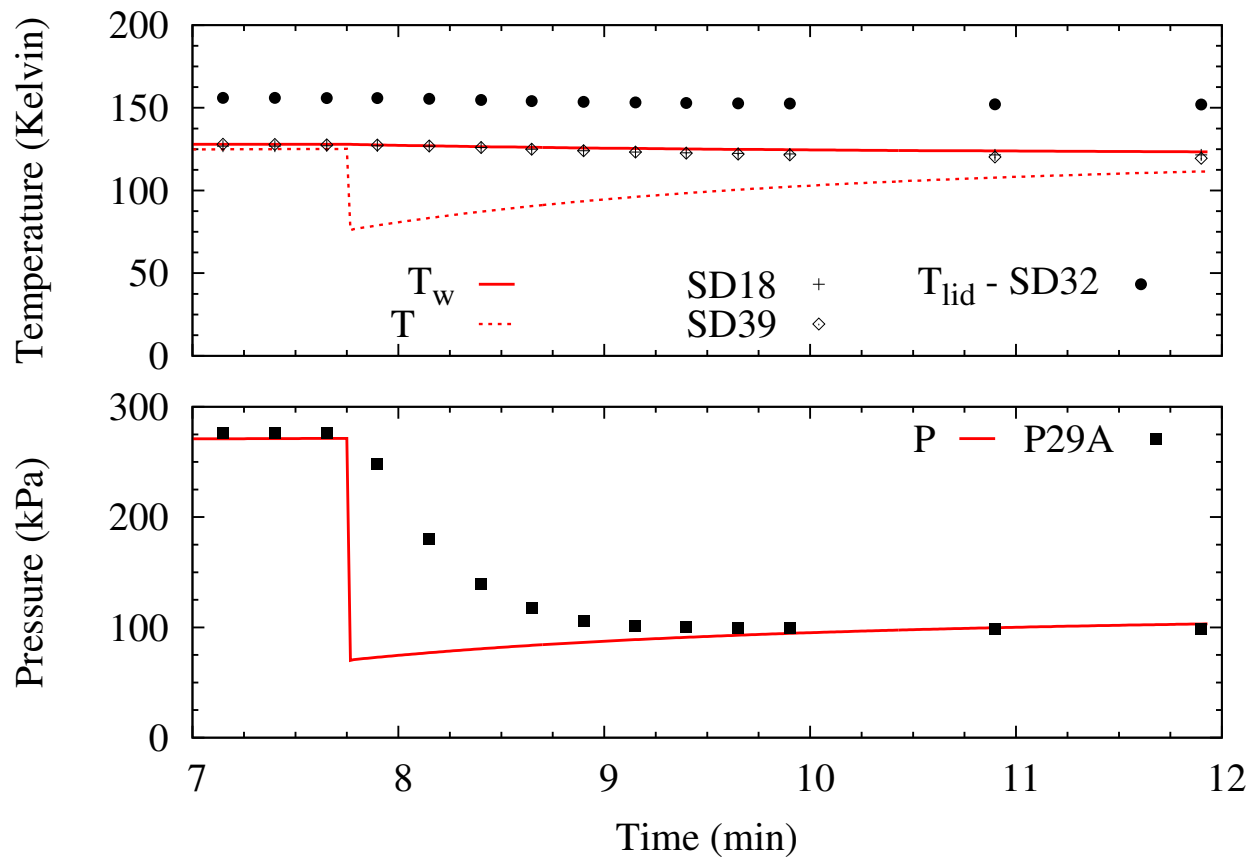

Figure 3.20: Total mass vented is $1.490 \mathrm{~kg}$ of $2.6 \mathrm{~kg}$ injected.

An instantaneous drop in pressure and temperature accompany the instantaneous vent. The temperature and pressure profiles eventually recover under the influence of natural convection effects but the characteristic transient behavior of the real venting process is lost.

Fig 3.21 illustrates the venting process when broken up into several smaller vent and hold processes where the mass per sub-vent, $m_{o}$, decreases with time. 

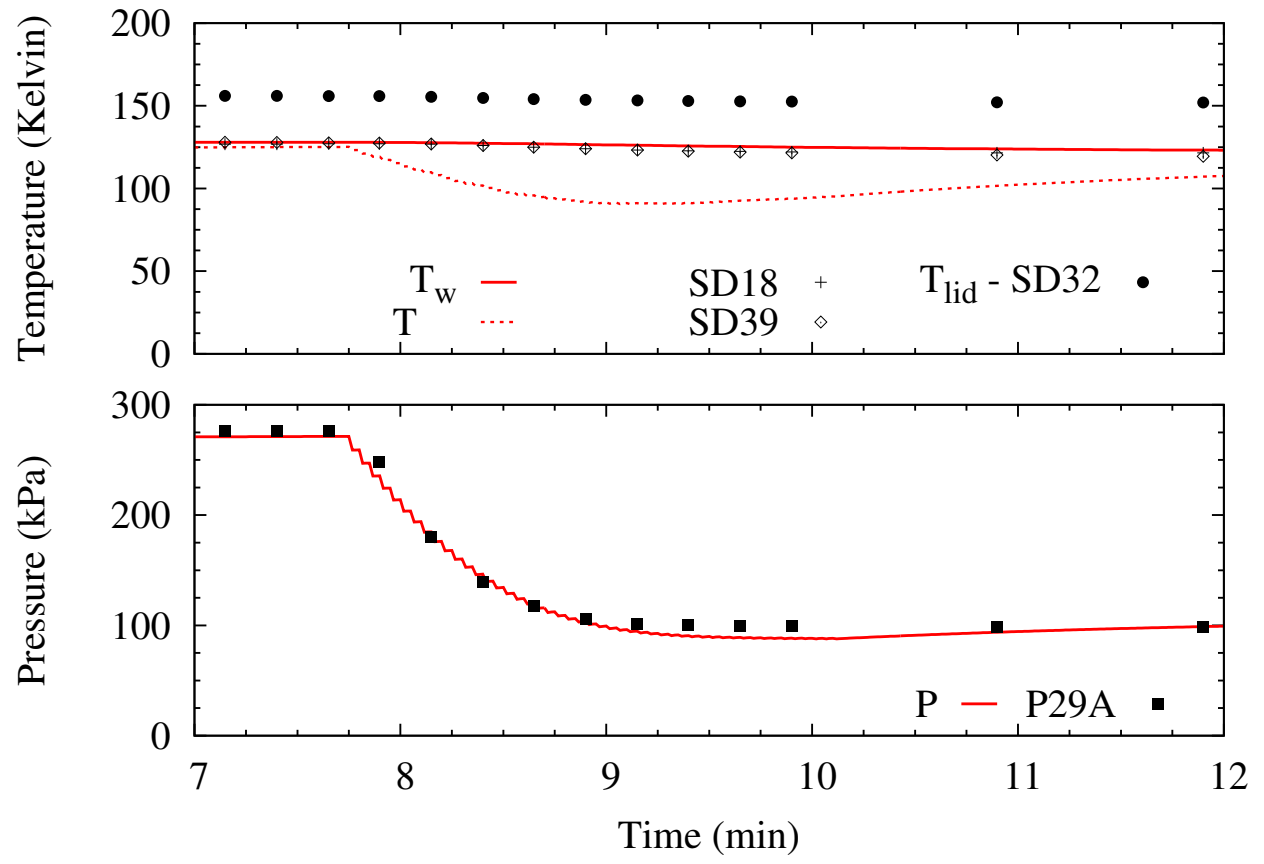

Figure 3.21: Transition over $152 \mathrm{sec}$ with $m_{o}^{1}=0.08 \mathrm{~kg}$. Total mass vented is $1.490 \mathrm{~kg}$ of $2.8 \mathrm{~kg}$ injected.

Isentropic mass removal cools the vapor remaining in the tank and the hold phase permits heat transfer between the vapor and tank wall. Mass removal occurs over small time scales relative to heat transfer and, for all intents and purposes, is instantaneous. Modeling the vent process in this fashion, as a parent vent divided into smaller sub-vents, enables the transfer of mass and heat to be simulated as pseudo-instantaneous. The inherent flexibility in this approach allows any type of vent process to be modeled.

Fig 3.22 details how the vent mass decreases with time during the parent vent process. 


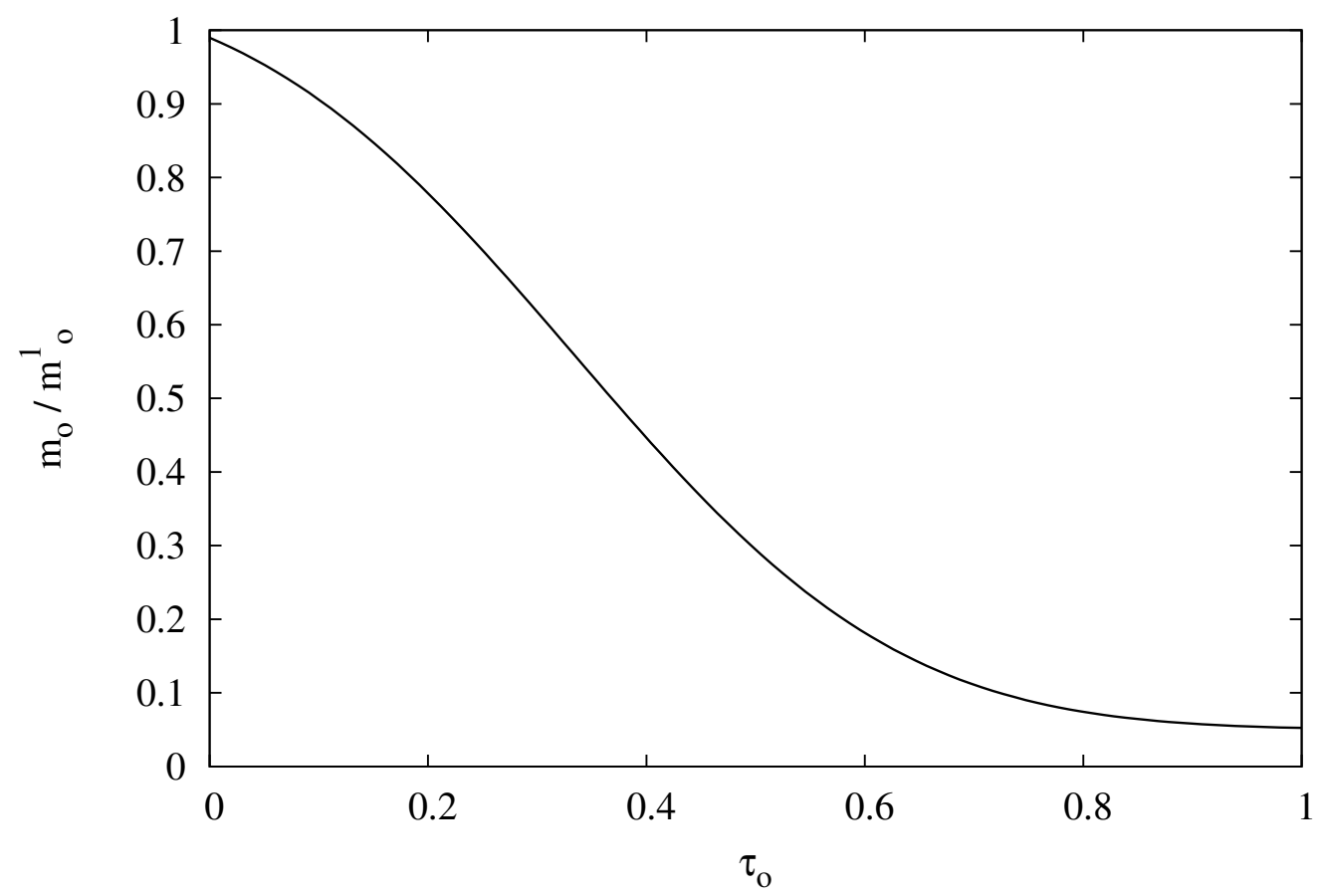

Figure 3.22: Decaying behavior imposed on $m_{o}$ during the vent process.

The scaling factor $m_{o}^{1}$ enables control over the rate that mass is vented. Holding all other vent parameters constant, increasing $m_{o}^{1}$ will allow more mass to be removed for each sub-vent as shown in Fig 3.23. 


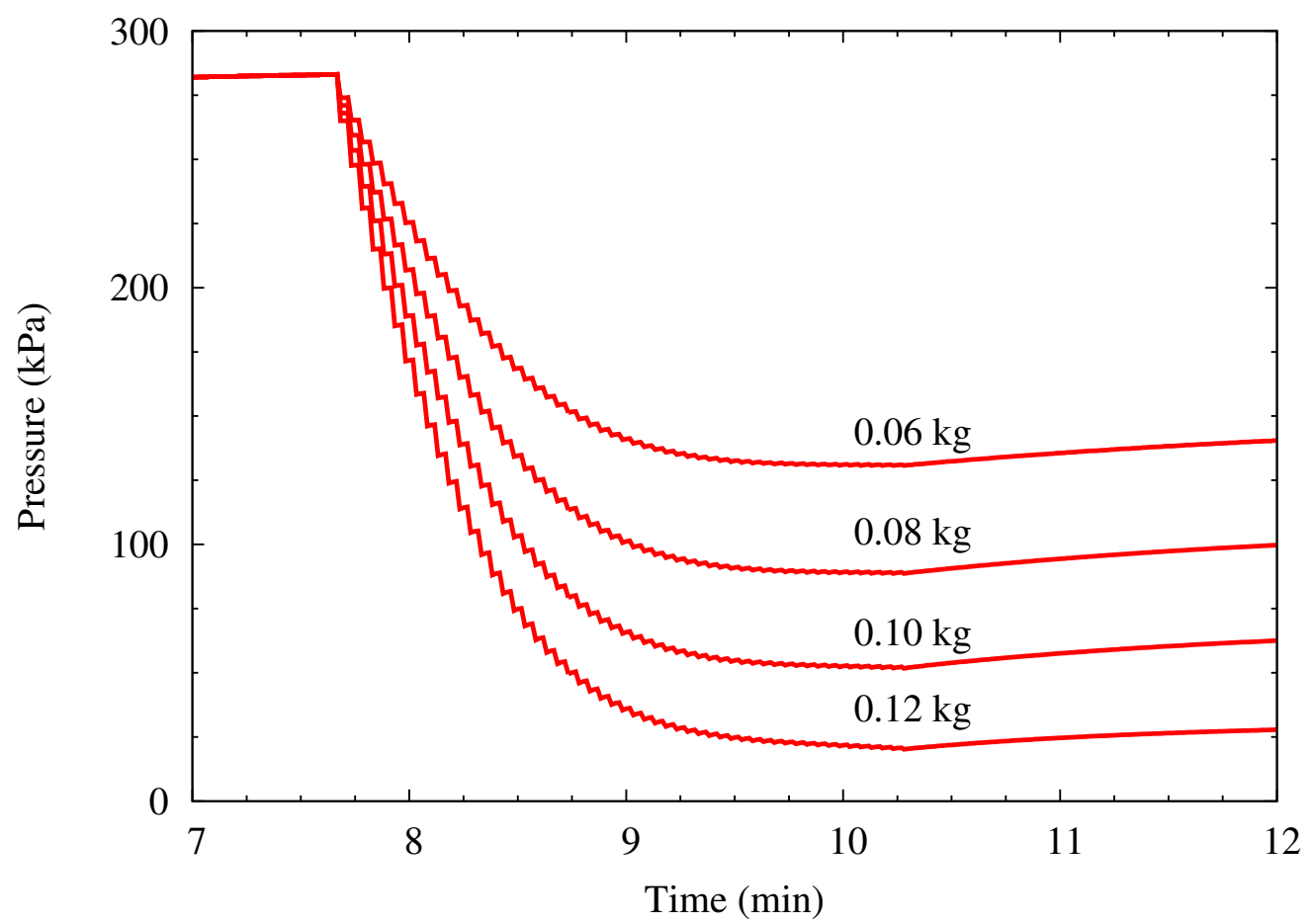

Figure 3.23: Example of parent vent behavior when varying $m_{o}^{1} . \quad N_{\text {sub-vent,max }}=53$, $\Delta t=1.0 \mathrm{~s}, N_{d t / s u b-v e n t}=3 \mathrm{~s}$. The value next to each plot represents the $m_{o}^{1}$ constant.

The amount of mass vented across a parent vent and the duration of the parent vent are decoupled. The behavior of the vent in terms of mass vented is controlled by $m_{o}^{1}$ while the heat transfer and, consequently, duration of the parent vent is controlled by $N_{\Delta t / s u b-v e n t}$.

Increasing $N_{\Delta t / s u b-v e n t}$ will increase the duration of the parent vent and has no effect on amount of mass vented. $N_{\Delta t / s u b-v e n t}$ is directly proportional to the amount of heat transferred during the parent vent. This effect is shown in Fig 3.24. 


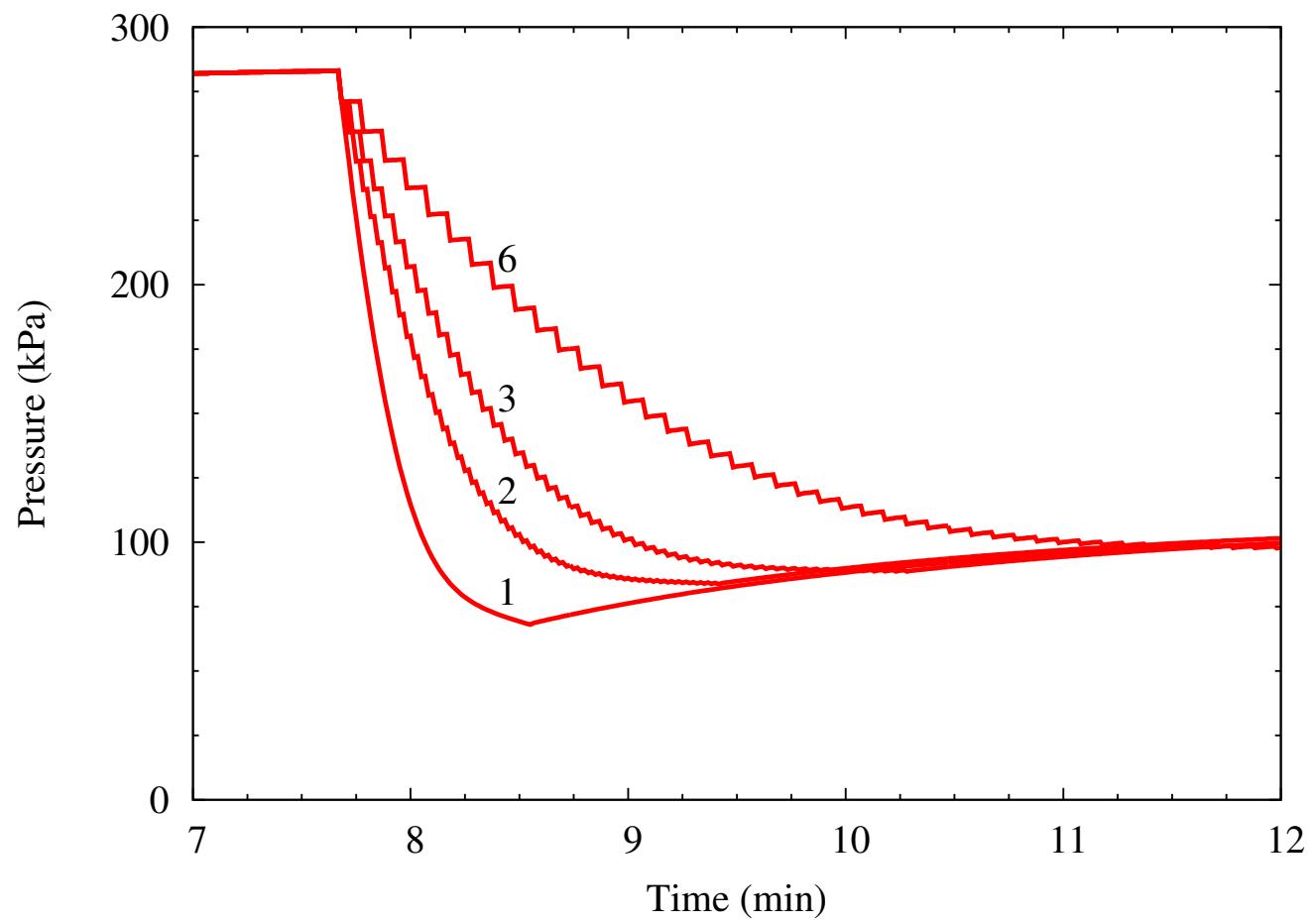

Figure 3.24: Example of parent vent behavior when varying the duration of the sub-vents. $N_{\text {sub-vent, } \max }=53, \Delta t=1.0 \mathrm{~s}, \mathrm{~m}_{o}^{1}=0.08$. The value next to each plot represents the number of time steps , $N_{\Delta t / \text { sub-vent }}$, of each sub-vent.

Fig 3.25 provides a simple example of how the mass inside the tank behaves during a vent. 


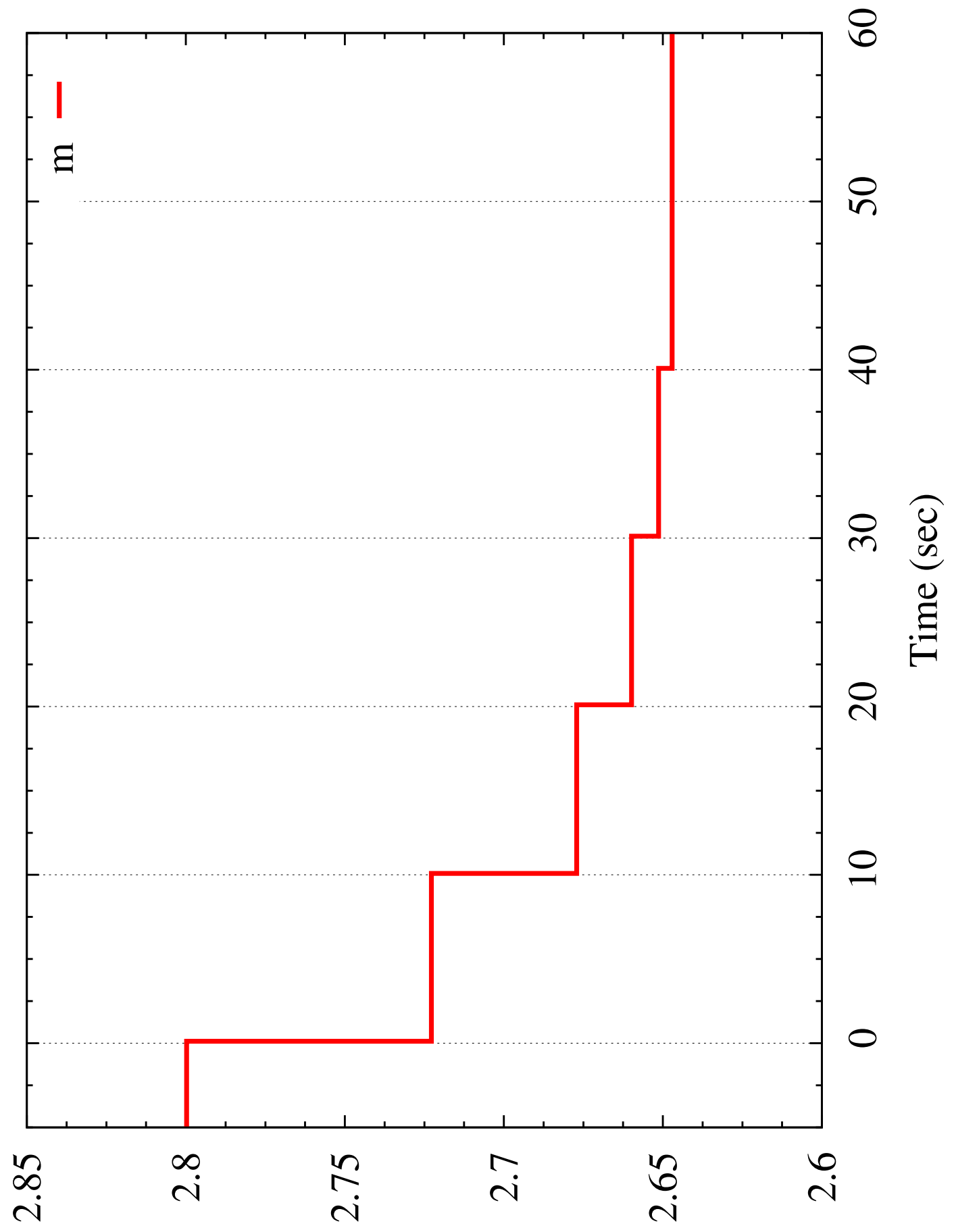

(8̊) YUR L U! SSEW

Figure 3.25: One parent vent modeled as 5 sub-vents. $m_{i}=2.8 \mathrm{~kg}, N_{\text {sub-vent,max }}=5$, $N_{\Delta t / \text { sub-vent }}=10, \Delta t=1.0 \mathrm{~s}, \mathrm{~m}_{o}^{1}=0.10$. 


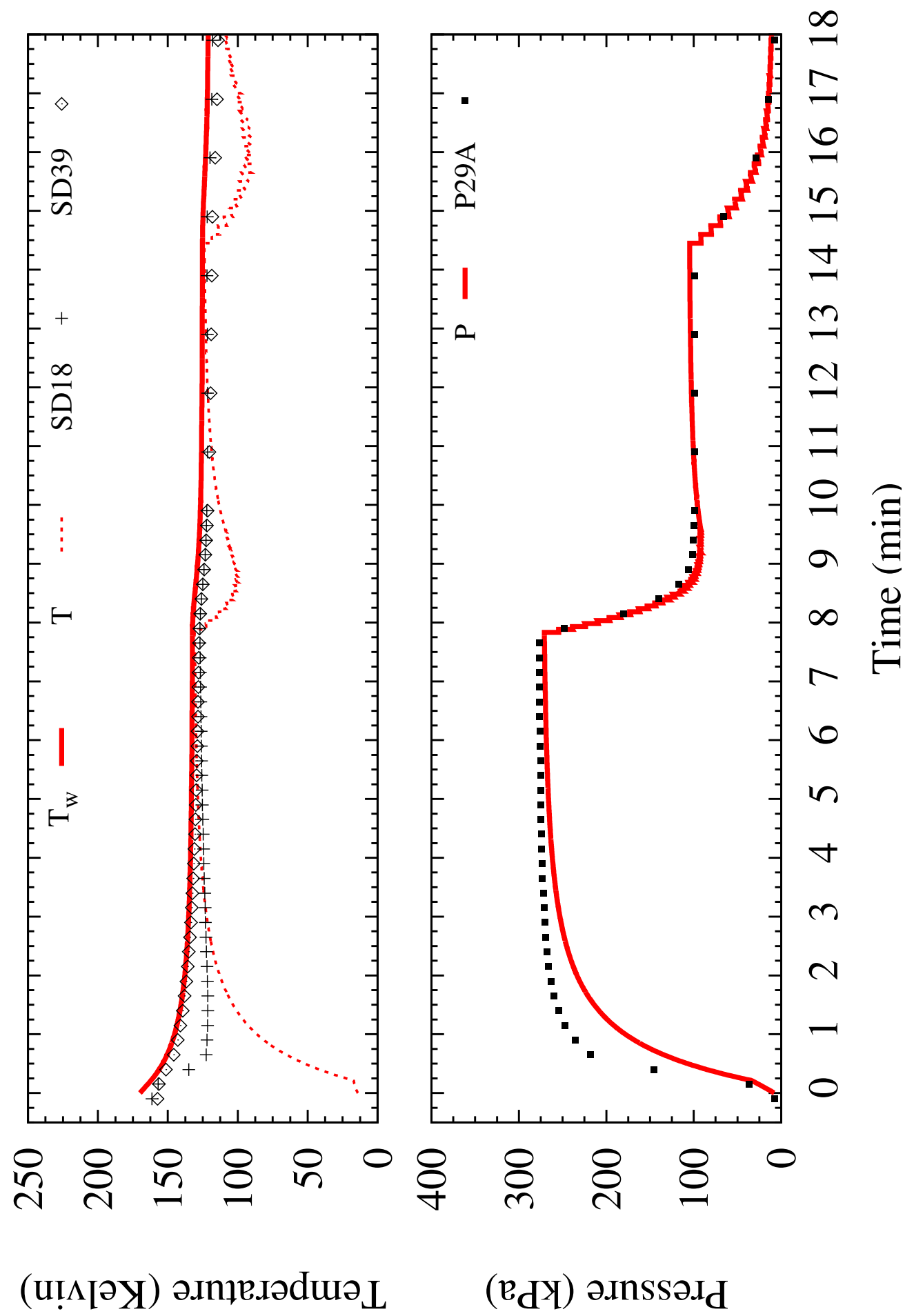

Figure 3.26: Simulation behavior for cycle 5 as a result of only natural convection effects. 


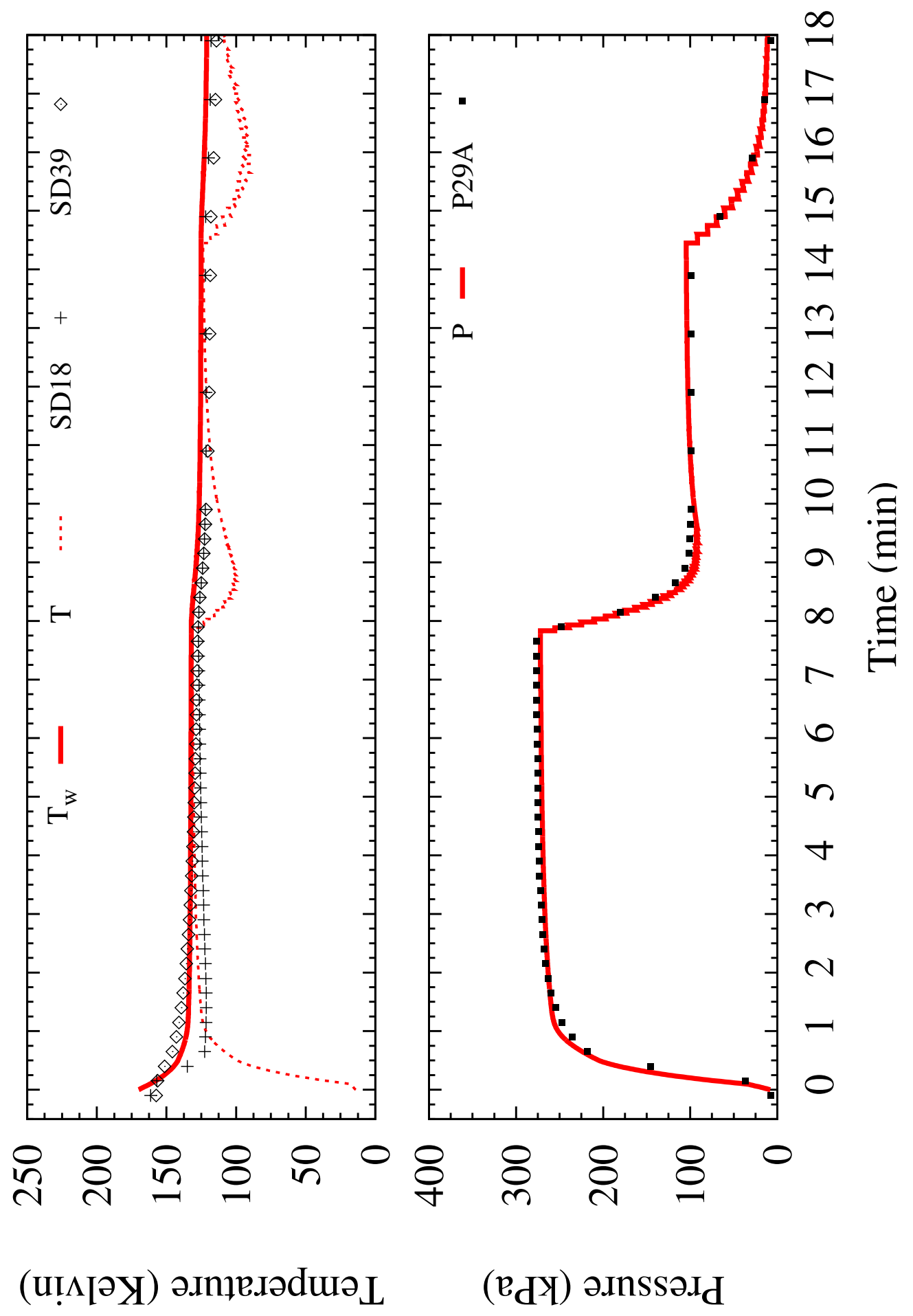

Figure 3.27: A complete model of cycle 5 data from [8]. 


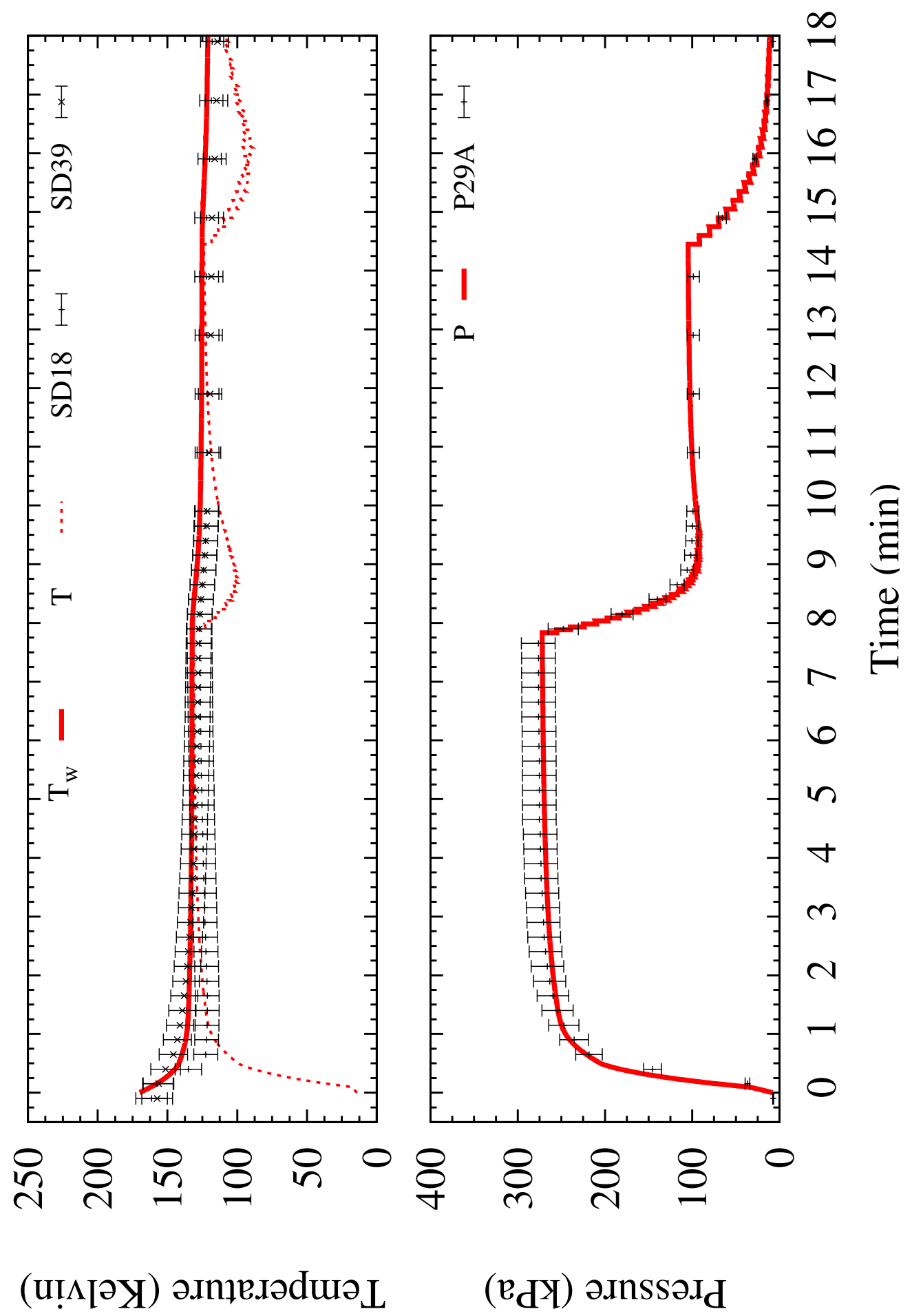

Figure 3.28: Simulation results compared to cycle 5 data with $7 \%$ error bars. 


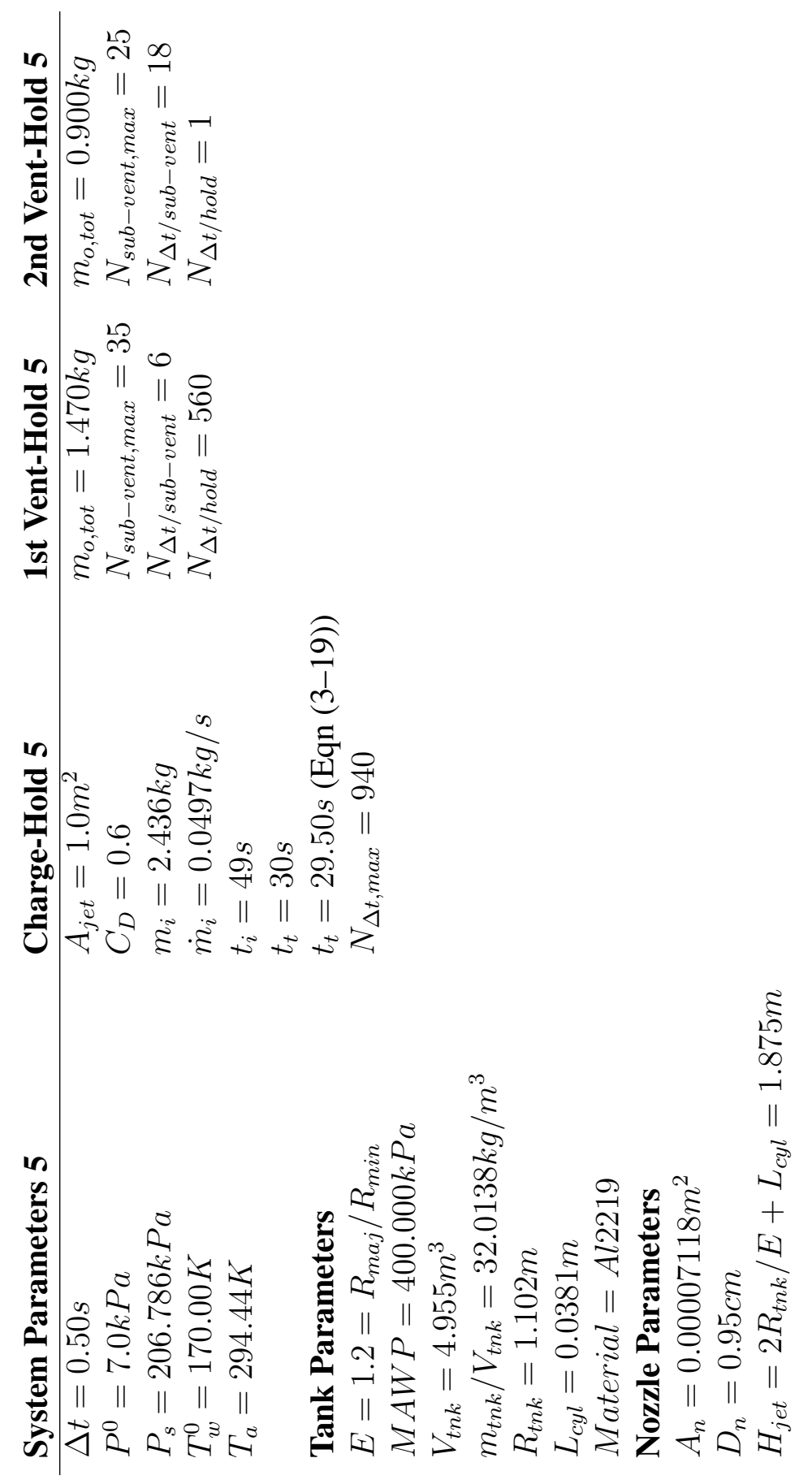

Table 3.4: Specified Model Inputs for Simulation of Cycle 5. 


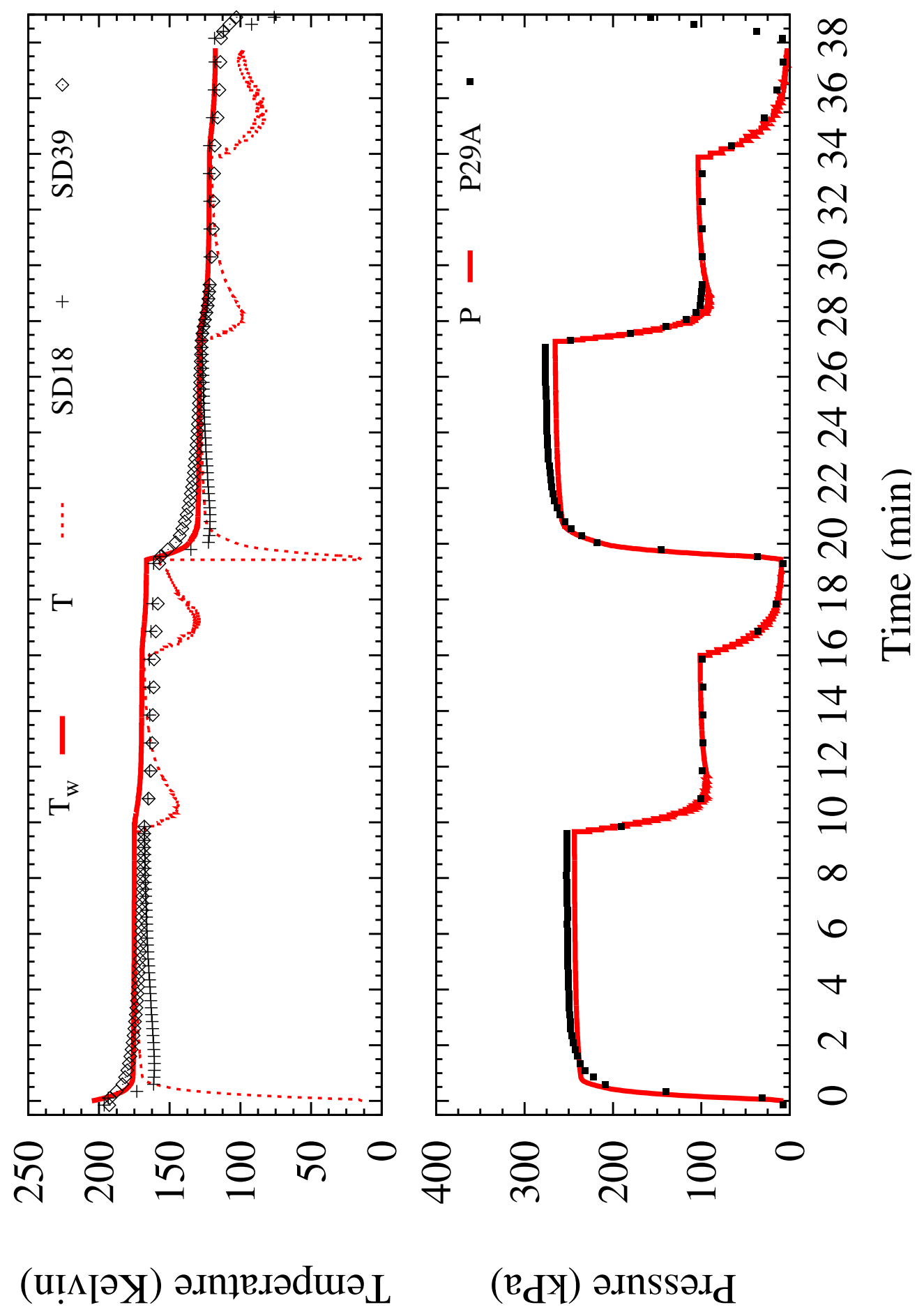

Figure 3.29: A complete model of cycle 4 and cycle 5 data from [8]. 


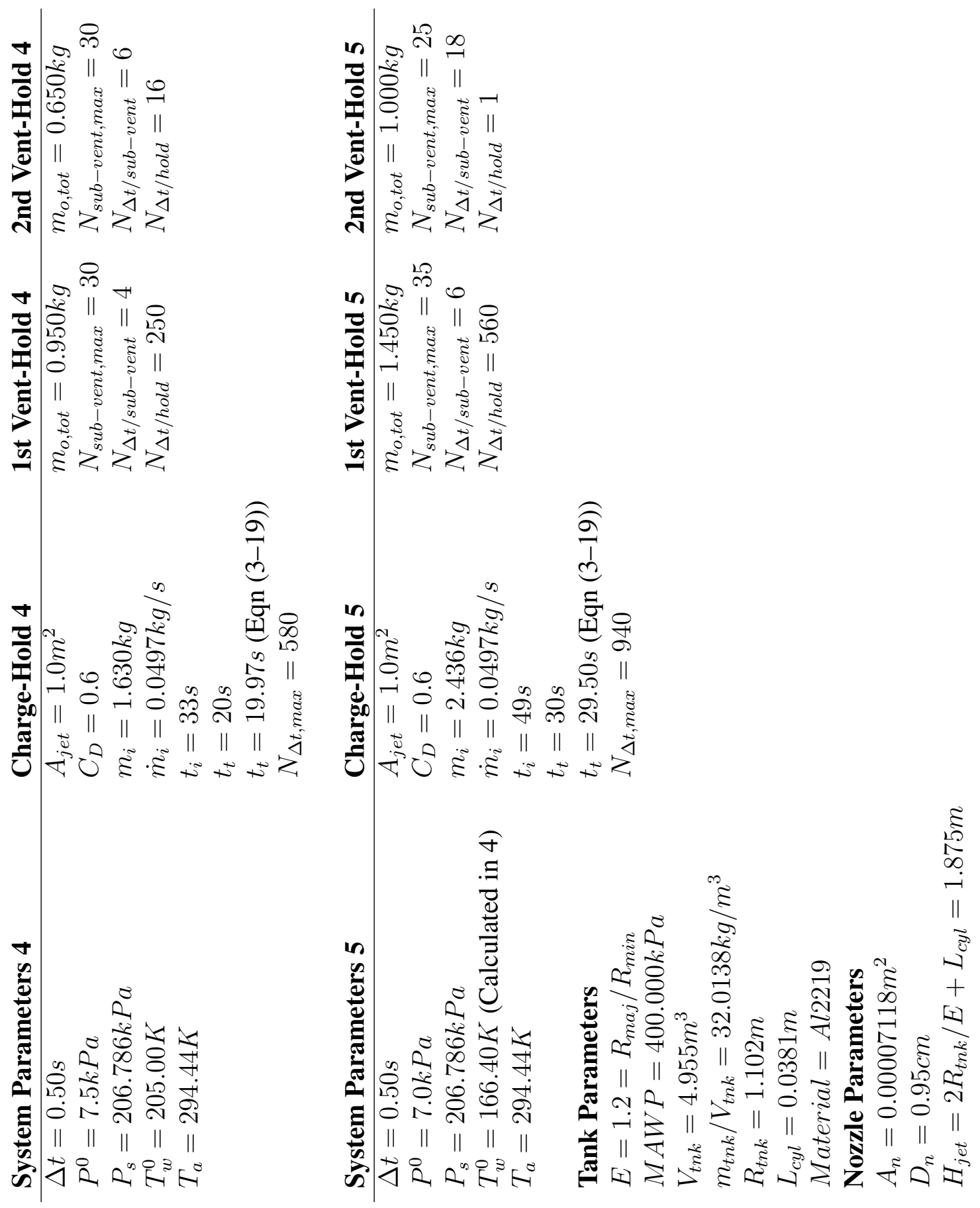

Table 3.5: Specified Model Inputs for Simulation of Cycle 4 followed by Cycle 5. 


\section{Chapter 4}

\section{Conclusions}

\begin{tabular}{l|rrr} 
& CHV Cycle 5 Experimental & Simulation & $\left|\frac{E-S}{E}\right| \cdot 100$ \\
& Parameters & Parameters & \\
\hline$T_{w}^{0}($ Kelvin $)$ & 165.000 & 170.000 & 3.03 \\
$P^{0}(k P a)$ & 6.755 & 7.000 & 3.55 \\
$P_{s}(k P a)$ & 206.786 & 206.786 & 0.00 \\
$m_{i}(k g)$ & 2.236 & 2.436 & 8.94 \\
$V_{t n k}\left(m^{3}\right)$ & 4.955 & 4.955 & 0.00 \\
$m_{t n k} / V_{t n k}\left(k g / m^{3}\right)$ & 30.137 & 32.014 & 6.23
\end{tabular}

Table 4.1: Comparison of cycle 5 experimental parameters to cycle 5 simulation parameters.

A flexible and robust analytical model capable of capturing the thermodynamic behavior of the Charge-Hold Vent process is developed and verified against experimental data and found to agree within 7\%. 85 fluid property models are available through REFPROP, 3 models for specific heat as a function of temperature account for the tank wall's thermodynamic behavior, and 9 combinations of endcap shapes account for the area and volume characteristics of the tank. A patching function is used to account for the transition between jet impingement and natural convection heat transfer regimes. Another patching function is imposed on the venting behavior of the tank contents. The two patching functions enable quick modifications to the code's behavior depending on hardware features and characteristics. Each venting procedure is divided into smaller vent-hold cycles to sim- 
ulate simultaneous heat and mass transfer. Mass removal is modeled as instantaneous and isentropic which enables the code to simulate cyclic venting schema, a $\mathrm{CHV}$ technique that makes additional cooling available through expansion of the tank contents. Characteristics are specified for each simulated parent vent with the option of a performing a hold phase between parent vents. The code's development is based on a single experimental CHV cycle but possesses the ability to model an entire $\mathrm{CHV}$ procedure, composed of multiple cycles.

The code may prove valuable in developing experimental ground tests of future cryogenic chilldown procedures as well as guidance for developing a $\mathrm{CHV}$ chilldown procedure with non-cryogenic fluids. The CHV technique enables efficient use of the working fluid's cooling potential, and is a valuable technique where strict hardware and system mass requirements determine the use of simplistic cooling and transfer solutions that do not rely on features enabled through adding hardware.

The model for cycle 5 converged to $\epsilon=1.0 E^{-6}$ in just under 1 minute of real time calculation on an Intel Atom CPU N550 @ $1.50 \mathrm{GHz}$ x 4 processor. Relaxing the convergence criteria to $\epsilon=1.0 \mathrm{E}^{-3}$ allowed the simulation to complete in $35 \mathrm{sec}$ with little to no change in behavior.

\subsection{Recommendations}

The code is built around a flexible solver routine that can handle additional governing equations that capture the subtle responses neglected in this first attempt at modeling $\mathrm{CHV}$. The inclusion of additional tank nodes would allow different regions of the tank wall to transfer heat at different temperatures. Conduction between these different regions is the next logical progression in expanding the codes capabilities.

Currently mass injection is instantaneous and the entirety of the charge mass is added to the tank at once. The capability to break up the injected mass into smaller sub-injections 
separated by short duration hold phases will improve the transient characteristics and reduce the presence of a two phase state. Additional models of injection heat transfer may be explored due to the expected change in behavior of the governing dimensionless groups.

The initial behavior of a chilldown cycle relies heavily on the heat transfer interactions between the injected fluid and the tank wall. The current model implements a turbulent jet Nusselt number correlation that was developed for the microelectronics industry with water and FC-77 as the working fluids. Impinging jet Nusselt number correlations developed from experiment with cryogenic fluids are not currently available. The simulation of cycle 5 saw Reynolds numbers of 2,700,000. Jiji found the jet impingement Nusselt number relation by experimenting in the range $10,000<R e<20,000$. The simulation's Reynolds number exceeded the experimental range used to determine the jet impingement correlation. Additionally, correlations developed at large ratios of separation distance to nozzle diameter (100-200) may improve the transient behavior of the code and reduce or avoid the need for a patching function.

The option for a control by pressure analysis would further improve the flexibility of the code. Currently the amount of mass injected and vented is specified. Two phase flow is nearly unavoidable during the chilldown process which decreases the accuracy of mass flow measurements. The actual execution of a $\mathrm{CHV}$ may implement a control by pressure scheme due to the simplification of the operation when compared to a control by mass approach.

The code was developed in accordance with experimental results for a single hydrogen CHV cycle. Validating the code's output with CHV data utilizing non-cryogenic fluids and cryogenic fluids other than hydrogen will help guide improvements to the methodology. 


\section{Bibliography}

[1] Chandler, F., Bienhoff, D., Cronick, J., and Grayson, G., "Propellant Depots for Earth Orbit and Lunar Exploration” AIAA-2007-6081, 2007.

[2] Zegler, F. and Kutter, B., "Evolving to a Depot-Based Space Transportation Architecture," 2010 AIAA Space Conference, August 30 - September 2, 2010, Anaheim, CA

[3] Kutter, B., "A Practical, Affordable Cryogenic Propellant Depot Based on ULAs Flight Experience," AIAA-2008-7644. 2008.

[4] Chato, D.J., "Technologies for Refueling Spacecraft On-Orbit," NASA TM-200021076, AIAA-2000-5107, November 2000.

[5] Johnson, W.L., "Thermal Performance of Cryogenic Multilayer Insulation at Various Layer Spacings," MS Thesis, Auburn University, 2007.

[6] Hecker, M., Mulqueen, J., Mcright, P., Meyer, M., et al, "NASA: Cryogenic Propellant Storage and Transfer (CPST) Technology Demonstration," Pre-Phase A, Government Point-of-Departure Concept Study, October 5, 2011.

[7] Vernon, R. M., and Brogan, J. J., "A Study of Cryogenic Container Thermodynamics During Transfer," Volume II Final Engineering Report, Lockheed Missiles and Space Co., K-14-67-3, 1967

[8] Chato, D.J., Sanabria, R., "Review and Test of Chilldown Methods for Space-Based Cryogenic Tanks," NASA TM-104458, AIAA Paper 91-1843, June 1991.

[9] Defelice, D.M., Aydelott, J.C., “Thermodynamic Analysis and Subscale Modeling of Space-Based Orbit Transfer Vehicle Cryogenic Propellant Resupply,' NASA TM89921, AIAA Paper 87-1764, June 1987.

[10] Honkonen, S.C., Bennett, F.O., Hepworth, H.K., "An Analytic Model for LowGravity Tank Chilldown and No-Vent Fill: The General Dynamics No-Vent Fill Program (GDNVF)," AIAA 91-1380, June 1991. 
[11] Chato, D.J., Moran, M.E., Nyland, T.W., "Initial Experimentation on the Nonvented Fill of a $0.14 \mathrm{~m}^{3}\left(5 \mathrm{ft}^{3}\right)$ Dewar With Nitrogen and Hydrogen," NASA TM-103155, AIAA Paper 90-1681, June 1990.

[12] Honkonen, S.C., Pietrzyk, J.R.,Schuster, J.R., “Analysis of Pulsed Injection for Microgravity Receiver Tank Chilldown," Advances in Cryogenic Engineering, Vol. 37, Part B, pgs 1265-1272, 1992.

[13] Pietrzyk, J.R, Honkonen, S.C., Schuster, J.R., "Fluid Motion Persistence in Micrograviy Receiver Tank Chilldown," International Astronautical Federation, IAF-90349, October 1990.

[14] Chato, D.J., "Analysis of the Nonvented Fill of a 4.96-Cubi-Meter Lightweight Liquid Hydrogen Tank," NASA TM-102039, August 1989.

[15] Chato, D.J., "Ground Testing for the No-Vent Fill of Cryogenic Tanks: Results of Tests for a 71 Cubic Foot Tank," NASA TM-106293, AIAA Paper 93-1967, June 1993.

[16] Touloukian, Y.S.,"Recommended Values of the Thermophysical Properties of Eight Alloys, Major Constituents and their Oxides," Nasa Order R-45, NBS Sub-Contract No. CST-7590, February 1966.

[17] Shatz, L.F.,"The effect of hair bundle shape on hair bundle hydrodynamics of nonmammalian inner ear hair cells for the full frequency range," Hearing Research Vol. 195, issues 1-2, September 2004.

[18] Lemmon, E.W., Huber, M.L., McLinden, M.O. NIST Standard Reference Database 23: Reference Fluid Thermodynamic and Transport Properties-REFPROP, Version 8.0, National Institute of Standards and Technology, Standard Reference Data Program, Gaithersburg, 2007.

[19] Oliphant, K., Webb, B.W., McQuay, M.Q., “An Experimental comparison of liquid jet array and spray impingement cooling in the non-boiling regime," Experimental Thermal and Fluid Sciences 18, 1998.

[20] Jiji L.M., Z. Dagan, "Experimental investigation of single phase multi-jet impingement cooling of an array of microelectronic heat sources," in Proceedings of the International Symposium of Cooling Technology for Electronic Equipment, The Pacific Institute for Thermal Engineering, HI, 1987, pp. 265.

[21] Martin, H., "Heat and Mass Transfer between impinging Gas Jets and Solid Surfaces, " in J.P. Hartnett and T.F. Irvine, Jr., Eds., Advances in Heat Transfer, Vol. 13, Academic Press, New York, 1977.

[22] Goldstein, R.J., E.M. Sparrow, and D.C. Jones, "Natural Convection Mass Transfer Adjacent to Horizontal Plates,' Int. J. Heat Mass Transfer, 16, 1025, 1973. 
[23] Lloyd J.R., Moran W.R., "Natural Convection Adjacent to Horizontal Surfaces of Various Planforms," ASME Paper 74-WA/HT-66, 1974.

[24] Incopera, F.P., DeWitt, D.P., "Fundamental of Heat and Mass Transfer $-4^{\text {th }}$ ed.," Wiley and Sons, 1996.

[25] Dominik, S . M., "Mixing Induced Condensation Inside Propellant Tanks," AIAA Paper 84-0514, January 1984.

[26] McAdams, W.H., Heat Transmissions, $3^{\text {rd }}$ ed., McGraw-Hill, New York, 1954, Ch. 7.

[27] Churchill, S.W., H.H.S. Chu, "Correlating Equations for Laminar and Turbulent Free Convection from a Vertical Plate," Int. J. Heat Transfer, 18, 1323, 1975.

[28] DeWitt, R.L., Boyle, R.J., "Thermal Performance of an Intergrated THermal Protection System for Long-Term Storage of Cryogenic Propellants in Space," NASA TN D-8320, May 1977.

[29] Horn, G., Thring, M.W., "Angle of Spread of Free Jets," University of Sheffield, Nature vol. 178 pg 205, July 28, 1956.

[30] Kutter, B., "Propellant Depots Made Simple," FISO Presentation, November 10, 2010.

[31] McLean, C., "Cryogenic Propellant Depot Design Concepts and Risk Reduction Activities," FISO Presentation, March 2, 2011.

[32] Mann, D., "LNG Materials and Fluids," National Bureau of Standards, Cryogenics Division First Edition, 1977.

[33] Touloukian, Y.S., Ho, C.Y., "Thermophysical Properties of Selected Aerospace Materials Part II: Thermophysical Properties of Seven Materials," 1976, p.39-46

[34] Pietrzyk, J.R., Honkonen, S.C., Schuster, J.R., "Fluid Motion Persistence in Microgravity Receiver Tank Chilldown," International Astronautical Federation Paper IAF90-349, October 1990.

[35] Bailey, W.J., et al., "Cryogenic On-Orbit Liquid Depot Storage, Acquisition and Transfer Satellite (COLD-SAT) Feasibility Studies," Martin Marietta Space Systems Co., NASA CR 185247, June 1990 . 